\title{
Development and Characterization of an Optically Tunable Dielectric Material
}

\author{
by \\ Nathan Jess, B.Eng.

\begin{abstract}
A thesis submitted to the
Faculty of Graduate Studies and Research

in partial fulfillment of the requirements for the degree of
\end{abstract}

Master of Applied Science in Electrical Engineering

Ottawa-Carleton Institute for Electrical and Computer Engineering

Department of Electronics

Carleton University

Ottawa, Ontario

September, 2010

(C) Copyright

Nathan Jess, 2010 
Library and Archives

Canada

Published Heritage

Branch

395 Wellington Street

Ottawa ON K1A ON4

Canada
Bibliotheque et

Archives Canada

Direction du

Patrimoine de l'édition

395 , rue Wellington

Ottawa ON K1A ON4

Canada
Your file Votre référence
ISBN: $978-0-494-71514-7$
Our file Notre référence
ISBN: $978-0-494-71514-7$
NOTICE:

The author has granted a nonexclusive license allowing Library and Archives Canada to reproduce, publish, archive, preserve, conserve, communicate to the public by telecommunication or on the Internet, loan, distribute and sell theses worldwide, for commercial or noncommercial purposes, in microform, paper, electronic and/or any other formats.

The author retains copyright ownership and moral rights in this thesis. Neither the thesis nor substantial extracts from it may be printed or otherwise reproduced without the author's permission.
AVIS:

L'auteur a accordé une licence non exclusive permettant à la Bibliothèque et Archives Canada de reproduire, publier, archiver, sauvegarder, conserver, transmettre au public par télécommunication ou par l'Internet, prêter, distribuer et vendre des thèses partout dans le monde, à des fins commerciales ou autres, sur support microforme, papier, électronique et/ou autres formats.

L'auteur conserve la propriété du droit d'auteur et des droits moraux qui protège cette thèse. $\mathrm{Ni}$ la thèse ni des extraits substantiels de celle-ci ne doivent être imprimés ou autrement reproduits sans son autorisation.
In compliance with the Canadian Privacy Act some supporting forms may have been removed from this thesis.

While these forms may be included in the document page count, their removal does not represent any loss of content from the thesis.
Conformément à la loi canadienne sur la protection de la vie privée, quelques formulaires secondaires ont été enlevés de cette thèse.

Bien que ces formulaires aient inclus dans la pagination, il n'y aura aucun contenu manquant. 


\section{Abstract}

The ever increasing use of wireless devices has caused a need for devices that can operate over a range of frequencies and conditions. This can be accomplished by using tunable circuits that utilize a tunable dielectric material. This work proposes a new optically tunable dielectric material that consists of a transparent polymer mixed with particles of cadmium sulfide, which are photoconductive.

A resonant characterization apparatus was developed to measure the microwave properties of optically sensitive films deposited on glass cover slips. The apparatus was a modification of the characterization apparatus known as the split-post dielectric resonator.

A potential method to simulate the properties of a heterogeneous material is presented. The validity of the simulations were confirmed by comparing them to Bruggeman's effective medium theory and percolation theory.

The characterization apparatus developed in this work was used to measure the complex permittivity of the polymer-cadmium sulfide films. The shift in dielectric constant of the films with illumination was at least $9 \%$. The dielectric loss tangent of the materials also increased by up to 0.085 , from 0.063 to 0.148 .

The optically tunable dielectric material shows a shift in dielectric constant that may make it comparable with ferroelectric materials. The opportunity exists to increase the change in the dielectric constant and decrease the change in the dielectric loss tangent with further optimization. 


\section{Acknowledgments}

I would like to express my gratitude to both my supervisors, Dr. Langis Roy and Dr. Barry Syrett, for providing their time and guidance. I would like to thank Dr. Steven McGarry for making the films, and for being available for consultations. I would also like to thank Nagui Mikhail for the providing servers for computationally intensive tasks and for assisting in obtaining equipment for measurements. I am grateful to many other members of the department of electronics for their assistance during this program.

Additionally, I would like to acknowledge the financial support provided by NSERC.

Finally, I would like to thank my close friends and family for their continued support. 


\section{Table of Contents}

Abstract

Acknowledgments

Table of Contents $\quad$ v

List of Tables $\quad$ ix

List of Figures $\quad$ xi

Nomenclature $\quad$ xvi

1 Introduction 1

1.1 Tunable Materials and Modern Communications . . . . . . . . . . . 1

1.2 Objectives of the Thesis .................... 2

1.3 Thesis Outline ...................... 2

2 Material Properties 4

2.1 Introduction . . . . . . . . . . . . . . . . 4

2.2 Permittivity ........................ 4

2.2.1 Electric Polarization . . . . . . . . . . . 6

2.2.2 Permittivity of Composite Materials . . . . . . . . . 17

2.3 Photoconduction ........................ 22 


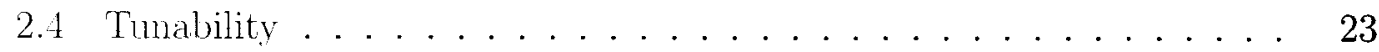

2.4 Ferroelectric Materials . . . . . . . . . . . 25

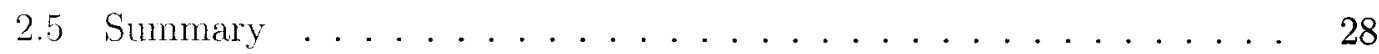

3 Optically Tunable Dielectric Material Analysis 30

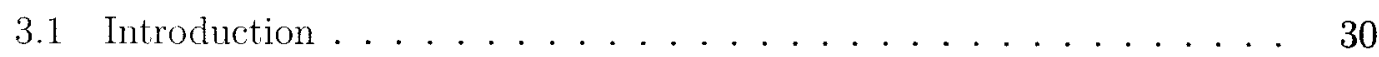

3.2 Theoretical Observations . . . . . . . . . . . . . . 31

3.3 Numerical Analysis . . . . . . . . . . . . . . . . . . 35

3.4 Conclusions ...................... 46

4 Microwave Material Characterization Theory $\quad 47$

4.1 Introduction . . . . . . . . . . . . . . 47

4.2 Free Space Transmission-Reflection . . . . . . . . . . . . . . 48

4.3 Resonant Perturbation Theory . . . . . . . . . . . . . 50

4.4 Dielectric Resonators . . . . . . . . . . . . . . . . . 53

4.5 Split-Post Dielectric Resonator (SPDR) . . . . . . . . . . . 56

4.6 Single-Post Dielectric Resonator(SiPDR) . . . . . . . . . . . . . 58

4.7 Unloaded Q Measurements Using a Vector Network Analyzer . . . . . 59

4.8 Summary .............................. 60

5 Novel Open Single Post Dielectric Resonator (OSiPDR) 62

5.1 Introduction . . . . . . . . . . . . . . . 62

5.2 Design of Cavity . . . . . . . . . . . . . . . 64

5.2 .1 Determination of Cavity Specifications .......... 64

5.2 .2 Simulation Setup and Simplification . . . . . . . 66 66

5.2 .3 Sizing of Cavity . . . . . . . . . . . . . 69

5.3 Material Property Extraction ................ 75 
5.4 Accuracy . . . . . . . . . . . . . . 76

5.5 Tuning and Verification of Cavity . . . . . . . . . . 79

5.5.1 Adjustment of Simulation Parameters. . . . . . . . . . 80

5.5 .2 Analysis of Empty Cavity . . . . . . . . . . . . . 81

5.5 .3 OSiPDR with Glass Substrates ............ 84

5.6 Conclusions . . . . . . . . . . . . . . . . . . 89

6 Experimental Material Characterization $\quad 90$

6.1 Introduction . . . . . . . . . . . . . . . 90

6.2 Material Preparation . . . . . . . . . . . . . . . 9 90

6.3 Microwave Material Property Characterization . . . . . . . . . . . . 92

6.4 Optical Material Property Characterization . . . . . . . . . . 97

6.5 Summary . . . . . . . . . . . . . . . . . . 99

7 Analysis and Discussion of Findings $\quad 100$

7.1 Introduction . . . . . . . . . . . . . . . 100

7.2 Optical Properties of Films . . . . . . . . . . . . . . 100

7.3 Expected Dielectric Constant ................ 101

7.4 Permittivity Changes with Optical Illunination . . . . . . . . 102

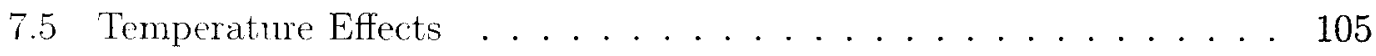

7.6 Appropriateness of an Effective Dielectric Constant . . . . . . . 109

7.7 Comparison to Ferroelectric Materials . . . . . . . . . . . . . 109

7.8 Simulating the Composite Material . . . . . . . . . . . . 110

8 Conclusions and Recommendations $\quad \mathbf{1 1 1}$

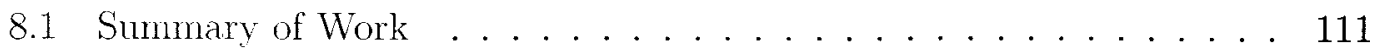

8.2 Further Work . . . . . . . . . . . . . . . . . 112 


\section{List of Tables}

2.1 Comparison of ferroelectric materials . . . . . . . . . . .

3.1 Effect of conductivity and volume fill fraction of the filler on $\epsilon_{\text {eff }}^{\prime}$ and dielectric loss tangent (The properties of the host matrix are $\epsilon_{r}^{\prime}=$ $3, \tan \delta=0.001) \ldots \ldots \ldots \ldots \ldots$

3.2 Effect of $\epsilon_{r}^{\prime}$ and conductivity of the filler on $\epsilon_{\text {eff }}^{\prime}$ and dielectric loss tangent (The properties of the host matrix are $\epsilon_{r}^{\prime}=3, \tan \delta=0.001$ ) .

3.3 Effect of dielectric constant of host and conductivity of filler on $\epsilon_{\text {eff }}^{\prime}$ and dielectric loss tangent (The properties of the host matrix are $\epsilon_{r}^{\prime}=$ $3, \tan \delta=0.001) \ldots \ldots \ldots \ldots \ldots$

5.1 Properties of dielectric resonator and support . . . . . . . . . 66

5.2 Characteristics of cavity with and without the lid . . . . . . 81

5.3 Characteristics of empty cavity .............. 83

5.4 Characteristics of substrates before material depositing ( $\mathrm{t}$ is the thickness of the glass $\ldots \ldots \ldots \ldots$. . . . . . . . . . . 85

5.5 Characteristics glass cover slip S1 ( $\mathrm{t}$ is the thickness of the glass) . . 85

5.6 Characteristics glass cover slip $\mathrm{S} 2$ ( $\mathrm{t}$ is the thickness of the glass) . . 86

5.7 Characteristics glass cover slip S3 ( $\mathrm{t}$ is the thickness of the glass) . . . 86

6.1 Results from measuring sample a9 (polymer and doped CdS) . . . . . 94

6.2 Results from measuring sample a10 (polymer and doped CdS) . . . . 95 
6.3 Results from measuring sample a11 (polymer and doped CdS) . . . . 95

6.4 Results from measuring sample a12 (polynner and undoped CaS) . . . 96

6.5 Results from measuring sample a13 (polymer and undoped CdS) . . . 96

6.6 Results from measuring sample a14 (polymer and undoped CdS) . . . 97 


\section{List of Figures}

2.1 Relaxation time of each type of polarization [1] . . . . . . 8

2.2 (a) Molecule orientated parallel to field; (b) molecule orientated perpendicular to field; (c) molecule randomly orientated [ ] . . . . . . . 9 9

2.3 Example of molecule without (a) and with (b) a permanent dipole

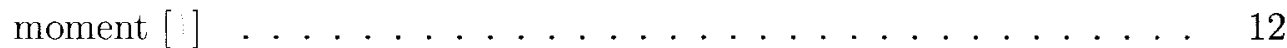

2.4 Parallel metal plate capacitor with two different conductive dielectrics [ ] 14

2.5 Effect of electric field on polarization of a ferroelectric material $(a) T \ll$ $T_{c},(\mathrm{~b}) T<T_{c}$, and $(\mathrm{c}) T>T_{c}[\mathrm{l}] \ldots \ldots 16$

2.6 Temperature dependence of complex permittivity of a ferroelectric material []$\ldots \ldots \ldots \ldots \ldots \ldots$

2.7 Section of 2 D resistor network f. . . . . . . . . . . 19

2.8 Example of percolation with a square lattice when each space is filled randomly with a probability of $60 \%[] \ldots \ldots 20$

2.9 Effect of ellipsoidal axial ratio (M) of filler particle on percolation threshold. [] ........................... 21

2.10 Effect of particle size ( $R$ is radius) of filler particle on percolation threshold []$\ldots \ldots \ldots \ldots 21 \ldots \ldots \ldots$. . . . . . . . . . . . .

2.11 Typical response of ferroelectric materials [ ] . . . . . . . . 26

2.12 Properties of $\mathrm{SrTiO}_{3}$ at $10 \mathrm{GHz}[] \ldots \ldots 26$ 
2.13 Dielectric constant and loss tangent of $\mathrm{Pb}_{0} \cdot 3 \mathrm{Sr}_{0} \cdot 7 \mathrm{TiO}_{3}$ films at $1 \mathrm{MHz}[] 27$

2.14 Tomperature and bias dependence of ferroelectric capacitors at $10 \mathrm{GHz}$ (a) $120 \mathrm{~K}(\mathrm{~b}) 200 \mathrm{~K}(\mathrm{c}) 275 \mathrm{~K}(\mathrm{~d}) 300 \mathrm{~K}[\mathrm{l} \ldots \ldots . \ldots 27$

3.1 Effect of the conductivity of the filler on $\epsilon_{\text {eff }}^{\prime}$ and dielectric loss tangent 33

3.2 Effect of the dielectric constant of the filler on $\epsilon_{\text {eff }}^{\prime}$ and dielectric loss tangent $\ldots \ldots \ldots \ldots \ldots \ldots$

3.3 Effect of the dielectric constant of the host on $\epsilon_{\text {eff }}^{\prime}$ and dielectric loss

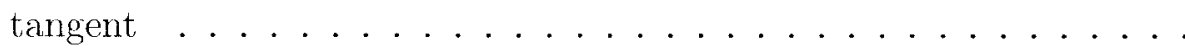

3.4 Setup used to simulate heterogeneous materials (Top and bottom has perfect magnetic boundary condition, while sides have perfect electric conductor boundary condition) . . . . . . . . . 36

3.5 Shape used to approximate a sphere . . . . . . . . . . . . . . . 37

3.6 Material with a $5 \%$ volume fill fraction . . . . . . . . . . 37

3.7 Effect of conductivity and volume fill fraction of filler on $\epsilon_{e f f}^{\prime}$ (The properties of the host matrix are $\left.\epsilon_{r}^{\prime}=3, \tan \delta=0.001\right) \ldots . \ldots 40$

3.8 Effect of conductivity and volume fill fraction of filler on dielectric loss tangent (The properties of the host matrix are $\epsilon_{r}^{\prime}=3, \tan \delta=0.001$ ) .

3.9 Effect of $\epsilon_{r}^{\prime}$ and conductivity filler on $\epsilon_{\text {eff }}^{\prime}$ (The properties of the host matrix are $\left.\epsilon_{r}^{\prime}=3, \tan \delta=0.001\right) \ldots \ldots \ldots$

3.10 Effect of $\epsilon_{r}^{\prime}$ and conductivity filler on dielectric loss tangent (The properties of the host matrix are $\left.\epsilon_{r}^{\prime}=3, \tan \delta=0.001\right) \ldots \ldots$

3.11 Effect of dielectric constant of host and conductivity of filler on $\epsilon_{\text {eff }}^{\prime}$ (The properties of the host matrix are $\left.\epsilon_{r}^{\prime}=3, \tan \delta=0.001\right) \quad \ldots$

3.12 Effect of dielectric constant of host and conductivity of filler on dielectric loss tangent (The properties of the host matrix are $\epsilon_{T}^{\prime}=3, \tan \delta=$

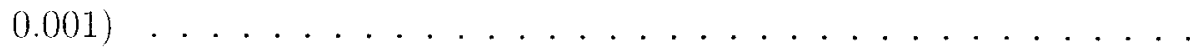


3.13 Dependence of $c_{\text {eff }}^{\prime}$ on volune filler fraction (filler particles have a conductivity of $1000 \mathrm{~S} / \mathrm{m}) \ldots \ldots \ldots \ldots$

3.14 Dependence of dielectric loss tangent on volume filler fraction (filler particles have a conductivity of $1000 \mathrm{~S} / \mathrm{m}) \ldots \ldots 44$

3.15 Dependence of $\epsilon_{\text {eff }}^{\prime}$ on volume filler fraction (filler particles have a conductivity of $10 \mathrm{~S} / \mathrm{m}) \ldots \ldots \ldots \ldots$

3.16 Dependence of diclectric loss tangent on volume filler fraction filler particles have a conductivity of $10 \mathrm{~S} / \mathrm{m}) \ldots \ldots \ldots$

4.1 Diagram of planar sample []$\ldots \ldots \ldots \ldots$

4.2 Small-object material perturbation. (a) Original material in cavity and (b) Perturbed cavity []$\ldots \ldots \ldots 51$

$4.3 T E_{01 \delta}$ mode of a dielectric puck resonator $[\ldots \ldots \ldots \ldots$

4.4 Effects of metallic wall and dielectric material on resonant frequency [ ] 55

4.5 Excitation of dielectric resonators []$\ldots \ldots \ldots 5$

4.6 Cross section of an SPDR $[\ldots] \ldots \ldots \ldots \ldots$

4.7 Cross section of SiPDR []$_{1} \ldots \ldots \ldots \ldots$

4.8 Input reflection coefficient of a resonant circuit with swept frequency [ ] 59

5.1 Cross section of OSiPDR (All components are cylindrical). . . . . 64

5.2 Models of resonant cavity . . . . . . . . . . . . . . 68

5.3 Magnitude of electric field in full cavity . . . . . . . . . . . . 69

5.4 Vector representation of electric fields in full cavity . . . . . . . 69

5.5 Dependence of the $\mathrm{Q}$ of resonator on distance DR is from base of cavity 70

5.6 Dependence of $Q$ of cavity on diameter of cavity . . . . . . . . . 70

5.7 Dependence of $\mathrm{Q}$ of cavity on cavity height . . . . . . . . . 71

5.8 Properties of cavity when a material with a dielectric constant varying from 2 to 100 and a dielectric loss of 0.001 is placed in the top holder 72 
5.9 Properties of cavity when a material with conductivity varying from $0.001 \mathrm{~S} / \mathrm{m}$ to $1000 \mathrm{~S} / \mathrm{m}$, dielectric constant of 3 , and dielectric loss tangent of 0.001 is placed in the top holder . . . . . . . . . . 72

5.10 Complete resonant cavity simulated in HFSS . . . . . . . . . . . 73

5.11 Dependence of cavity properties on dielectric constant of a $10 \mu \mathrm{m}$. thick

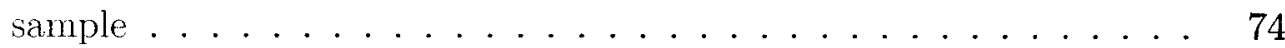

5.12 Dependence of cavity properties on dielectric loss of a $10 \mu \mathrm{m}$ thick sample 74

5.13 Effect of variances in the properties of the glass substrates on the shift in $f_{0}$ caused by the MUT . . . . . . . . . . . . . 77

5.14 Effect of uncertainty in thickness of MUT . . . . . . . . . 78

5.15 Full measurement apparatus (diameter of apparatus is $35 \mathrm{~mm}$ ) . . . 79

5.16 Parts of Measurement apparatus . . . . . . . . . . . . . . 80

5.17 Setup used to measure properties of OSiPDR $\ldots \ldots \ldots 8$

5.18 Dielectric constant of substrates before material is deposited on them $(t=160 \mu m) \ldots \ldots \ldots \ldots \ldots \ldots$

5.19 Dielectric loss of substrates before material is deposited on them $(t=$

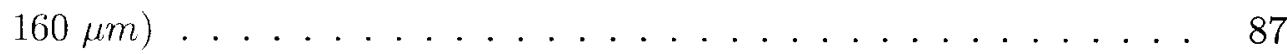

5.20 Dielectric constant of glass cover slips $(t=160 \mu \mathrm{m}) \ldots \ldots 8$

5.21 Dielectric loss of glass cover slips $(t=160 \mu m) \ldots \ldots . \ldots 8$

6.1 Glass cover slips with polymer-CdS deposited (scratch on leftmost one was made when measuring thickness) . . . . . . . . . . . . . 91

6.2 Spectrum of the lamp used (curve for 6333 is the bulb used) [ ] . . 93

6.3 Setup used to measure optical transmission . . . . . . . . . . . . . 97

6.4 Optical transmittance of polymer and doped CdS . . . . . . . 98

6.5 Optical transmittance of polymer and undoped CaS . . . . . . . . . 99 
7.1 Effect of illumination on the diclectric constant of all samples at $30^{\circ} \mathrm{C}$ $(t=11 \mu \mathrm{m}$. Error bars represent $t=9 \mu \mathrm{m}$ samples $) \ldots . . . . .103$

7.2 Effect of illumination on the dielectric loss tangent of all samples at $30^{\circ} \mathrm{C}(t=11 \mu \mathrm{m}$. Error bars represent $t=9 \mu \mathrm{m}$ samples $) \ldots 103$

7.3 Change in dielectric constant with illumination . . . . . . . . . 104

7.4 Change in dielectric loss tangent with illumination . . . . . . . . . 104

7.5 Diclectric constant of samples at differcnt temperatures without illu-

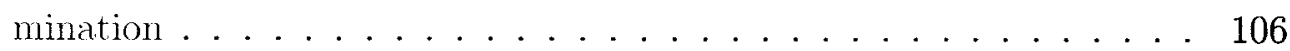

7.6 Dielectric loss tangent of samples at different temperatures without

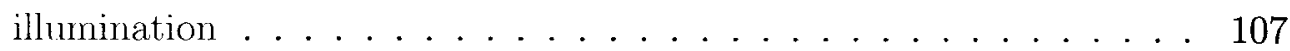

7.7 Change in dielectric constant of samples at different temperatures with

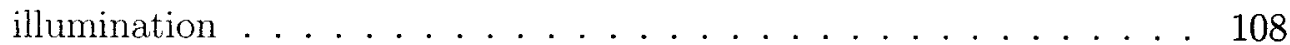

7.8 Change in dielectric loss tangent of samples at different temperatures with illumination . . . . . . . . . . . . . . 108 


\section{Nomenclature}

$\alpha$

$\alpha_{d}$

$\alpha_{e}$

$\alpha_{i}$

$\alpha_{o}$

$\alpha_{s}$

$\epsilon_{\circ}$

$\epsilon_{e f f}$

$\epsilon_{r}$

$\eta$

$\sigma$

$\sigma_{e f f}$

$\kappa$

$\lambda$

$\mu_{e}$

$\mu_{h}$

$\mu_{r}$

$\tau_{r}$

$\chi$
Average electronic polarization

Space charge polarization

Electronic polarization

Atomic polarization

Orientational polarization

Spontaneous polarization

Permittivity of free space

Effective permittivity of a material

Relative permittivity

Quantum efficiency

Electrical conductivity $(\mathrm{S} / \mathrm{m})$

Effective conductivity

coupling coefficient

Wavelength

Electron mobility

Hole mobility

Relative permeability

Electron-hole pair recombination time

Electric susceptibility 


$\begin{array}{ll}\text { DR } & \text { Dielectric resonator } \\ f_{\circ} & \text { Resonant frequency } \\ \text { I } & \text { Intensity of light } \\ \text { MUT } & \text { Material under test } \\ \text { OSiPDR } & \text { Open Single Post Dielectric Resonator } \\ \mathrm{P} & \text { Electric polarization } \\ \mathrm{PEC} & \text { Perfect electric conductor } \\ \mathrm{PMC} & \text { Perfect magnetic conductor } \\ Q & \text { Quality factor } \\ Q_{\circ} & \text { Unloaded quality factor } \\ \text { SiPDR } & \text { Single Post Dielectric Resonator } \\ \text { SPDR } & \text { Split Post Dielectric Resonator } \\ \mathrm{t} & \text { thickness } \\ \text { tan } \sigma & \text { Loss tangent of material } \\ V_{C} & \text { Volume cavity } \\ V_{S} & \text { Volume sample } \\ \text { VFF } & \text { Volume fill fraction } \\ \text { VNA } & \text { Vector network analyzer }\end{array}$




\section{Chapter 1}

\section{Introduction}

\subsection{Tunable Materials and Modern Communica- tions}

The use of wireless devices has increased drastically over the last decade. This increased use has caused a need for more efficient devices with increased functionality. Many of these devices must function over a range of frequencies and conditions, which can be achieved using two different techniques. The device can be designed to function over a range of frequencies and conditions, such as a broadband amplifier. Alternatively the device can be designed to be tunable, such as a narrowband amplifier whose center frequency can be shifted. Designing a circuit to operate over a range of frequencies usually comes at the cost of performance. However, if the circuit is made tunable, it can usually be designed to function with increased efficiency.

A wide variety of techniques have been utilized in order to make devices tunable [ - ]. Some of the more common techniques are electrical and mechanical tuning. Electrically tunable devices can complicate the device design and decrease performance due to the need for DC bias networks. The mechanically tunable devices 
have a relatively short lifespan or require maintenance. Another option is to tune optically. The optical tuning signal is applied without the need of the DC bias networks used in electrically tunable devices. Also, the optical tuning can be achieved without the need of moving parts that are used in mechanically tunable circuits. Additionally, optical tuning has the benefit that the control signal is isolated from the microwave signal being processed. In other words, the strength of the microwave should have relatively little effect on the properties of the optically tunable material. Optically tunable microwave circuits have been demonstrated previously [ , ], however a material with an optically tunable dielectric constant has not yet been reported.

\subsection{Objectives of the Thesis}

The primary overall objective of this work is to demonstrate that the dielectric constant of a material can be made optically tunable by combining an optically transparent polymer with photoconductive particles. In order to achieve this goal two specific goals are pursued. The first is to develop an apparatus to accurately characterize the microwave properties of the optically sensitive material. The second is to determine a possible method to predict the properties of an optically sensitive material.

\subsection{Thesis Outline}

Chapter 2 describes the dielectric properties of materials, photoconduction, and tunable circuits and materials. Chapter 3 comprises of the theoretical and numerical approaches used to describe the optically tunable dielectric material. Chapter 4 is 
concerned with microwave material characterization and the theory needed to understand the techniques used within this work. Chapter 5 discusses in detail the design and verification of the microwave material characterization apparatus used for this work. Chapter 6 gives results from measuring the properties of the proposed optically tunable material. Chapter 7 discusses the results obtained in Chapter 6. Chapter 8 gives a brief summary of the work done and gives direction for future work in the area of optically tunable dielectric materials. 


\section{Chapter 2}

\section{Material Properties}

\subsection{Introduction}

There are a few properties of materials that are important to understand for this work. First, it is important to understand what factors affect the complex permittivity of a material. A material's effective permittivity can be increased by incorporating conductive constituents into the material. Thus by adding a material with variable conductivity one can create a composite material that has a variable permittivity. If a material is photoconductive, its conductivity can be changed under optical illumination. The property of photoconductivity is explained following a discussion of permittivity. The chapter is concluded with a discussion on current tunable circuits and ferroelectric materials, which have an electrically variable dielectric constant.

\subsection{Permittivity}

Permittivity is a measure of a material's ability to reduce the total electric field inside the bulk of the material. This reduction of the electric field inside the material is accomplished by the displacement of charges that align with the electric field. This 
effect is known as electric polarization. When a homogeneous isotropic material has an electric field $\vec{E}$ applied to it the electric flux $\vec{D}$ is given by Equation (2.1) [ ]. The parameter $\vec{P}$ is the electric polarization of the material, and $\epsilon_{0}$ is the permittivity of free space.

$$
\vec{D}=\epsilon_{0} \vec{E}+\vec{P}
$$

If one assumes that the medium is also linear, then the electric polarization is related to the applied field by the following equation [ ]:

$$
\vec{P}=\epsilon_{0} \chi \vec{E}
$$

where, $\chi$ is the electric susceptibility of the material and is given by the following equation []:

$$
\chi=\frac{N \alpha}{\epsilon_{0}}
$$

where, $\alpha$ is the average electronic polarizability of the material and $N$ is the number of particles per unit volume. The complex permittivity of a material is represented by the following equation:

$$
\epsilon=\epsilon_{0}\left(\epsilon_{r}^{\prime}-j \epsilon_{r}^{\prime \prime}\right)=\epsilon^{\prime}-j \epsilon^{\prime \prime}
$$

The real part of the equation $\epsilon^{\prime}$ is related to the electric susceptibility of the material by $\epsilon_{r}^{\prime}=1+\chi$, while the imaginary part $\epsilon^{\prime \prime}$ accounts for the dielectric loss tangent in the medium. If the material has a conductivity $\sigma$ then the complex permittivity is given by:

$$
\epsilon=\epsilon_{0}\left(\epsilon_{r}-j \epsilon_{r}^{\prime \prime}-j \frac{\sigma}{\omega \epsilon_{0}}\right)
$$


For a conductive material the loss tangent is given by:

$$
\tan \delta=\frac{\omega \epsilon_{r}^{\prime \prime}+\sigma}{\omega \epsilon_{r}^{\prime}}
$$

The permittivity of a material is directly related to the electric polarization of a material. Thus in order to understand what affects permittivity, it is important to understand the different forms of polarization. The permittivity of a material can be increased when small volume fractions of a metallic material are added to an insulating material. This effect can be described by effective medium theories.

\subsubsection{Electric Polarization}

There are several different types of dielectric polarization. In order to understand each type of polarization, it is easiest to discuss the basic factors that affect the polarizability of a material. If the reader wishes to gain a more in-depth understanding of dielectric properties, then some excellent textbooks are: Dielectric Phenomena In Solids [ ], Electrical Properties of Polymers [ ], and Electrical Properties of Mate-

rials [ ]. The following material is condensed from Dielectric Phenomena in Solids, which is the most comprehensive of the three texts.

The permittivity of any type of homogeneous material is related to the average polarizability of all the particles in the material by the following equation:

$$
\epsilon_{r}=\frac{\epsilon}{\epsilon_{0}}=\left(1+\frac{N \alpha}{\epsilon_{0}}\right)
$$

where,

$\epsilon_{r}$ is the relative permittivity

$\epsilon$ is the permittivity 
$\epsilon_{0}$ is the permittivity of free space

$N$ is the density of molecules per unit volume

$\alpha$ is the average polarizability of the material

There are three types of polarization when dealing with electric fields much lower than inter-atomic fields (inter-atomic fields can be of the order of $10^{11} \mathrm{Vm}^{-1}$, while externally applied fields rarely exceed $10^{8} \mathrm{Vm}^{-1}[\mathrm{]})$, and for materials with negligible conductivity. The three types of polarization are electronic $\left(\alpha_{e}\right)$, atomic $\left(\alpha_{i}\right)$, and orientational polarization $\left(\alpha_{o}\right)$. When the conductivity of the material is no longer negligible, space charge polarization $\left(\alpha_{d}\right.$, which is broken into interfacial and hopping polarization) becomes important. Under special conditions some materials exhibit a type of polarization known as spontaneous polarization $\left(\alpha_{s}\right)$. The total polarizability of the material $\alpha$ combines all five contributions:

$$
\alpha=\alpha_{e}+\alpha_{i}+\alpha_{o}+\alpha_{d}+\alpha_{s}
$$

All types of polarization take a finite amount of time for the polarization to occur and each type of polarization has a force counteracting it. This means that each type of polarization will be frequency dependent and will have some loss. Figure 2.1 shows each type of polarization and the associated relaxation time.

The following sections discuss the different types of polarization. The discussion is only meant to give an idea of how each of the polarization mechanisms work. 


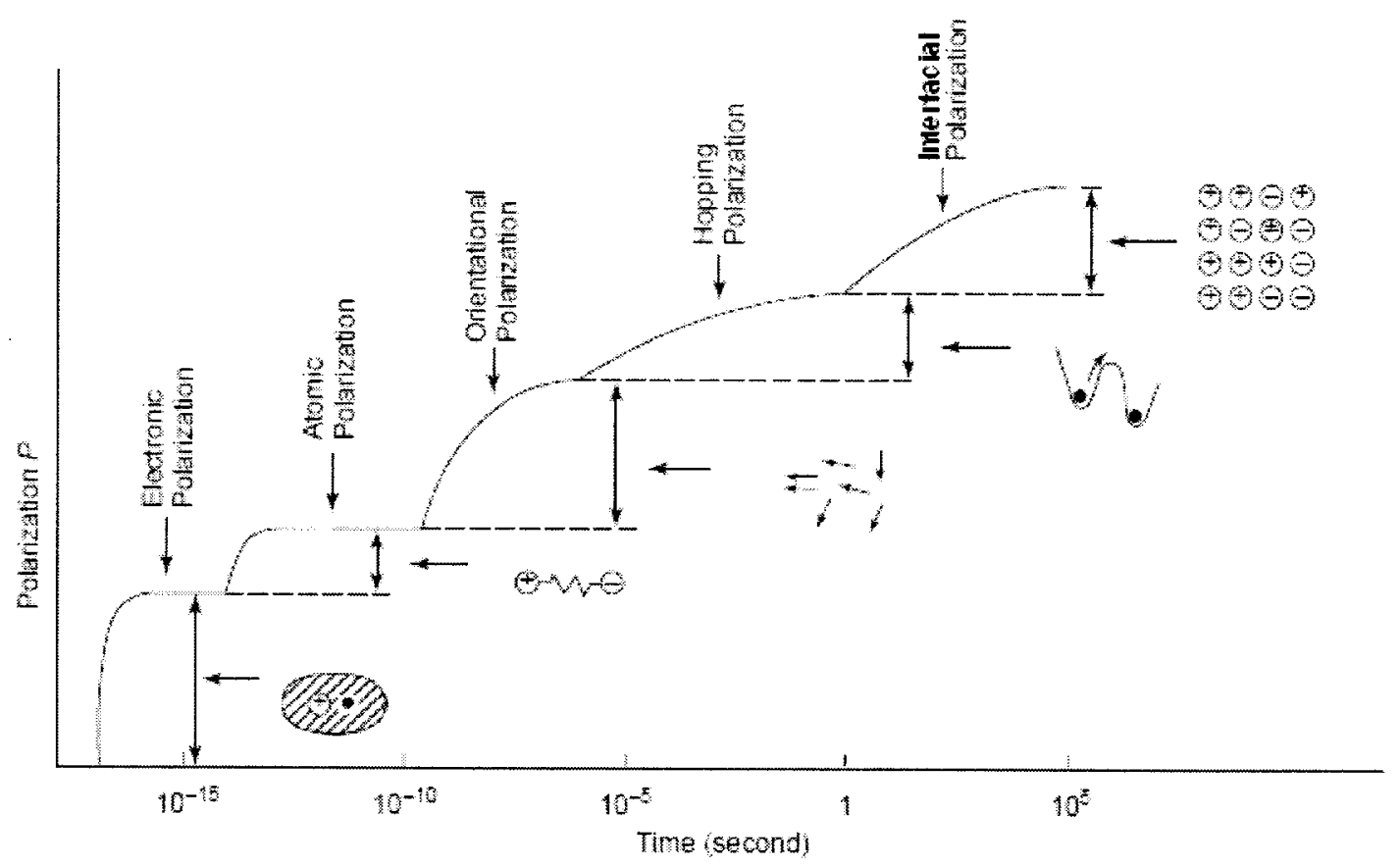

Figure 2.1: Relaxation time of each type of polarization []

\section{Electronic Polarization}

Electronic polarizability depends mainly upon the number of electrons in the outermost shells of the atoms that make up the material. The elements with full outer shells such as the noble gases have electronic polarizabilities lower than any other element. While the elements with single valence electrons have the highest polarizabilities. When calculating electronic polarization only the contribution of the outermost electrons are calculated because inner electrons provide negligible contributions. The electronic polarizability is effectively a measure of the displacement of the outer electron cloud when an electric field is applied. The easiest way to explain electronic polarizability is in terms of classical physics. The results obtained using classical approaches are very similar to the results obtained using quantum mechanics [ ]. 
In order to give an idea of the factors that affect electronic polarizability, the polarizability of a monoatomic gas is discussed. Calculating the polarizability assuming Bohr's model where electrons revolve around atoms in a circular orbit yields Equation (2.9) for the electronic polarizability.

$$
\alpha_{e}=\frac{(Z q)^{2}}{m_{e}\left(\omega_{0}^{2}-\omega^{2}\right)+j \beta \omega}
$$

where:

$Z$ is the number of electrons (typically the number in the outer shell of the atom) $q$ is the charge of an electron

$m_{e}$ is the mass of the electron

$\omega_{0}$ is the natural frequency of the atom

$\beta$ is the damping coefficient which represents the imaginary part of the permittivity

In the case that molecules are made up of more than one atom, the geometric shape will play an important role on $\alpha_{e}$. The orientation of the molecule in reference to the applied field will cause the polarizability of that molecule to change greatly.

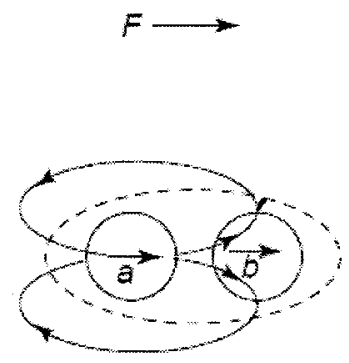

(a)

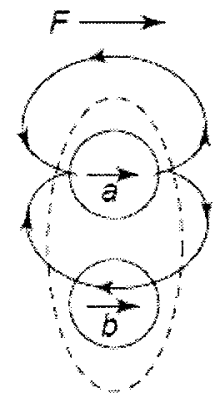

(b)

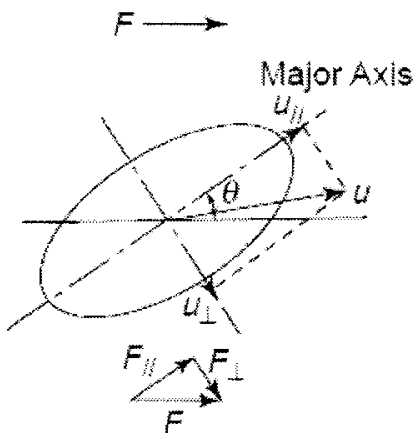

(c)

Figure 2.2: (a) Molecule orientated parallel to field; (b) molecule orientated perpendicular to field; (c) molecule randomly orientated [] 
When the molecule is oriented parallel to the applied field as shown in Figure 2.2(a) the polarizability of the material is given by:

$$
\alpha_{/ /}=\frac{2 \alpha_{e}}{1-2(R \sqrt{a b})^{3}}
$$

where:

$a b$ is the distance between the two atoms

$\alpha_{e}$ is the polarizability of a single atom

$R$ is the average orbital distance of the valence electrons

When the molecule is oriented parallel to the applied field, the polarizability of the two atom molecule is more than double the polarizability of the single atom. When the molecule is oriented perpendicular to the applied field as shown in Figure 2.2(b), the polarizability is given by:

$$
\alpha_{\perp}=\frac{2 \alpha_{e}}{1+2(R \sqrt{a b})^{3}}
$$

For the molecule being perpendicular to the field the polarizability is less the twice the polarizability of the single atom.

If the molecule is oriented in a random orientation as shown in Figure 2.2(c) then the polarizability will be between the values of perpendicular and parallel orientation. The equations given are a rough approximation because the complications of molecular interactions have been ignored. The time required for electronic polarization is about $10^{-15} \mathrm{~s}$, which means that electronic polarization is important right up to 
optical frequencies.

\section{Atomic Polarization}

A material that is composed of polyatomic molecules will have atomic polarization if there is a charge difference across the molecule. Consider a two atom molecule such as $\mathrm{NaCl}$. The sodium atom gives up one electron to the chloride atom $78 \%$ of the time, which makes the chloride atom negatively charged and the sodium atom positively charged. When an electric field is applied to this molecule, it will either expand or contract. This expansion or contraction of the molecule is known as atomic polarization. This type of polarization has a relaxation time of about $10^{-13}$ seconds. The atomic polarizability of a material with no inter-molecular interactions is:

$$
\alpha_{i}=\frac{(Z q)^{2}}{M_{r}\left(\omega_{0}^{2}-\omega^{2}\right)+j \beta \omega}
$$

where,

$Z$ is the number of electrons donated to the negative ion (eg. $Z=1$ for $\mathrm{NaCl}$ molecule)

$q$ is the charge of an electron

$M_{r}$ is the effective mass of the molecule

$\omega_{0}$ is the natural oscillation frequency

$\beta$ is the damping coefficient.

The equations for electronic and atomic polarizations are similar. This is because the electronic polarizability is the displacement of an electron cloud, while atomic polarizability is the displacement of the atoms in a molecule. 


\section{Orientational Polarization}

Orientational polarization can only occur in materials that possess permanent dipoles and have net dipole moment such as the molecule shown in Figure 2.3(b). The molecule shown in Figure 2.3(a) has no permanent dipole moment and will not contribute to orientational polarization.

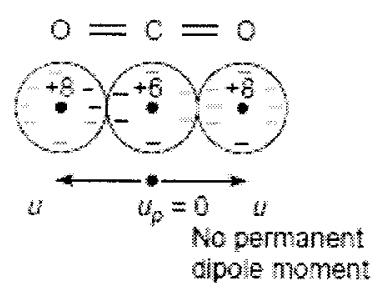

(a)

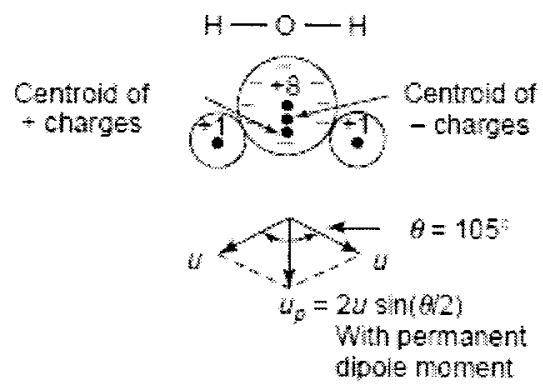

(b)

Figure 2.3: Example of molecule without (a) and with (b) a permanent dipole moment []

Since orientation polarization requires the movement of the entire molecule, it means that a significant amount of energy is required and the process is heavily temperature dependent. Orientational polarization decreases as temperature increases, because thermal agitation will tend to help put the molecules back into a random orientation. Since both atomic and electronic polarization are relatively temperature independent, the temperature can be varied to isolate the effects of orientation polarization. Many materials show a drastic change in permittivity when going from solid to liquid state because molecules cannot move easily in a solid. This type of 
polarization falls off rapidly in the $\mathrm{GHz}$ region so it has very little application at microwave frequencies.

\section{Space Charge Polarization}

Space charge polarization is a form of electric polarization, but it is associated primarily with mobile charges (electrons, holes, and ions). This type of polarization is only of importance once a material has a high density of free charge carriers. Space charge polarization occurs in materials that trap charge carriers in the bulk or at interfaces. Hopping polarization is a form of space charge polarization whereby charge carriers overcome a potential barrier and get trapped in a localized state. The probability of surmounting a potential barrier can be increased when an electric field is applied. Thus the applied field can cause a net displacement of charge which acts as a form of polarization. Interfacial polarization is another form of space charge polarization, and is very likely to occur in a materials made up of small conducting particles in an insulating host. Calculating the electric polarization using the theory of space charge polarization is a daunting task. Fortunately, there are numerous effective medium theories which deal with such heterogeneous mixtures and some of them are discussed in the next section. In order to give a better description of interfacial polarization, a relatively simple example of two dielectric materials between parallel metal plates as shown in Figure 2.4 is discussed. Material 1 has a conductivity $\sigma_{1}$, permittivity $\epsilon_{1}$, and thickness $d_{1}$. Material 2 has a conductivity $\sigma_{2}$, permittivity $\epsilon_{2}$, and thickness $d_{2}$ 


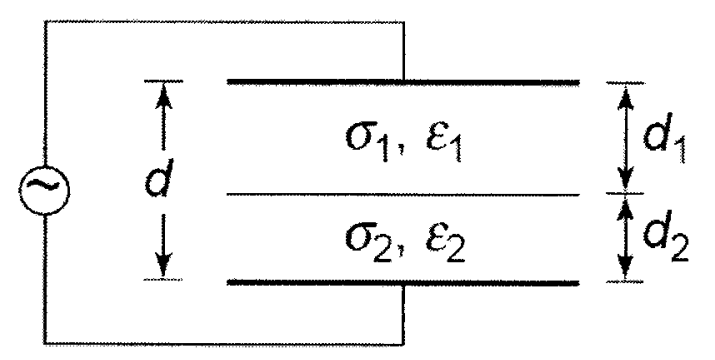

Figure 2.4: Parallel metal plate capacitor with two different conductive dielectrics [ ]

The following equation can be used to describe the effective permittivity $\left(\epsilon_{e f f}\right)$ and conductivity $\left(\sigma_{e f f}\right)[]$.

$$
\begin{gathered}
\tau_{1}=\frac{\epsilon_{1} \epsilon_{0}}{\sigma_{1}} \\
\tau_{2}=\frac{\epsilon_{2} \epsilon_{0}}{\sigma_{2}} \\
\tau=\epsilon_{0}\left(\frac{\epsilon_{1} d_{2}+\epsilon_{2} d_{1}}{\sigma_{1} d_{2}+\sigma_{2} d_{1}}\right) \\
\epsilon_{e f f}=\frac{1}{\epsilon_{0}}\left(\frac{\sigma_{1} \sigma_{2} d}{\sigma_{1} d_{2}+\sigma_{2} d_{1}}\right) \frac{\tau_{1}+\tau_{2}+\omega^{2} \tau_{1} \tau_{2} \tau-\tau}{1+\omega^{2} \tau^{2}} \\
\sigma_{e f f}=\left(\frac{\sigma_{1} \sigma_{2} d}{\sigma_{1} d_{2}+\sigma_{2} d_{1}}\right) \frac{1-\omega^{2} \tau_{1} \tau_{2}+\omega^{2} \tau_{1} \tau+\omega^{2} \tau_{2} \tau}{1+\omega^{2} \tau^{2}}
\end{gathered}
$$

Equation 2.15 describes the time required for interfacial polarization to occur. This equation shows that the frequency range in which this effect is significant is dependent on the permittivity, conductivity, and size of the materials. It should be noted that as conductivity increases the value of $\tau$ will decrease, which means that interfacial polarization can have a much smaller relaxation time than what was shown in Figure 2.1. The much smaller relaxation time means that interfacial polarization 
is significant right up to and including microwave frequencies.

\section{Spontaneous Polarization}

Spontaneous polarization is an effect that is associated with ferroelectric materials. Ferroelectric materials generally have a high dielectric constant and the permittivity is heavily dependent upon temperature and electric field [ ]. For a material to exhibit ferroelectric effects it must be crystalline in structure. The crystal is made up of unit cells and each of these cells will be electrically non-polar when the temperature is above the Currie temperature $\left(T_{c}\right)$. When the ferroelectric material is above $T_{c}$ the material is in the paraelectric state. When the temperature of the ferroelectric material goes below $T_{c}$ then the crystal goes from a non-polar to polar structure and the material is in the ferroelectric state. Each unit cell will carry an electric dipole moment when the temperature is below $T_{c}$ and will spontaneously align parallel to the dipole moment of the neighboring cells. The chain reaction that occurs is referred to as spontaneous polarization. The process of cells aligning with each other will eventually stop and each section of cells locally aligned with each other is referred to as a domain. Other domains will form throughout the material and be aligned randomly as long as no electric field is applied. When an electric field is applied, the domains will align in the direction of the field, and once the field is removed the polarization will remain. Since the domains do not randomize upon removal of the electric field the relation of polarization to the electric field will form a hysteresis loop [ ]. As the temperature of the material increases the hysteresis loop will be less pronounced as shown in Figure 2.5 


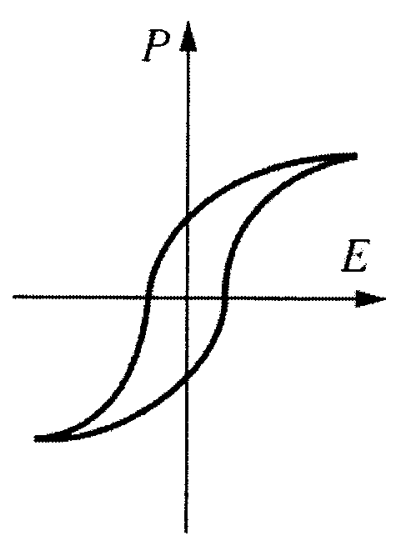

(a)

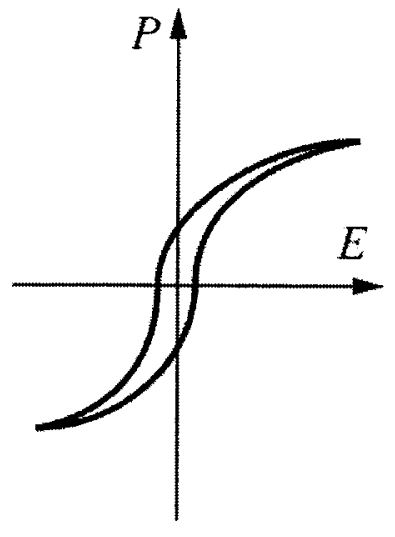

(b)

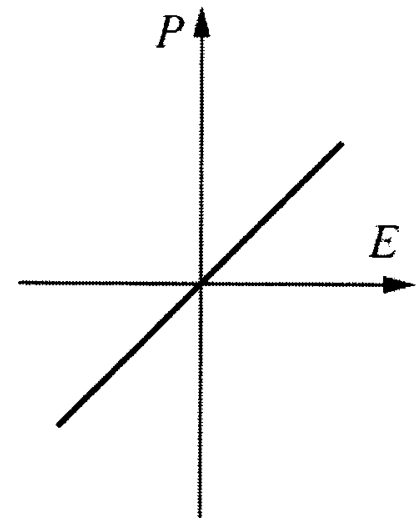

(c)

Figure 2.5: Effect of electric field on polarization of a ferroelectric material (a) $T \ll$ $T_{c},(\mathrm{~b}) T<T_{c}$, and $(\mathrm{c}) T>T_{c}[\mathrm{l}]$

Ferroelectric materials are generally not used in the ferroelectric state because the dielectric loss tangent is too high. The dependence of the complex permittivity of a ferroelectric material on temperature is shown in Figure 2.6.

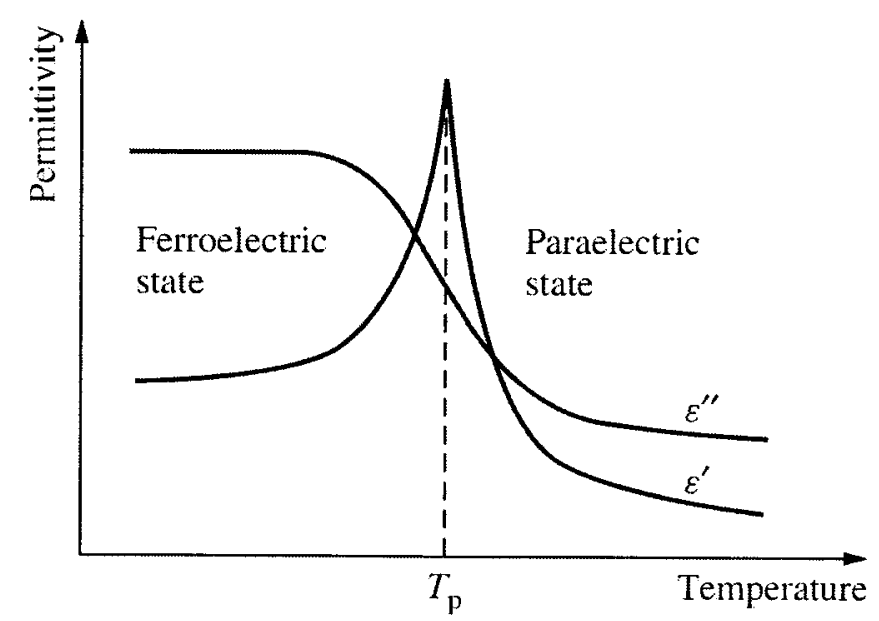

Figure 2.6: Temperature dependence of complex permittivity of a ferroelectric material [ ]

The dielectric constant of a ferroelectric material in the paraelectric state is dependent upon the electric field applied. The application of the electric field will cause 
the dielectric constant of the material to decrease [ ].

\subsubsection{Permittivity of Composite Materials}

There have been many theories that attempt to describe the bulk electrical properties of a heterogeneous material based upon the electrical properties and volume loadings of the constituents. There is a significant amount of literature on effective medium theories $[$,$] . This section describes the effective medium theory devel-$ oped by Bruggeman [ ]. Bruggeman's theory breaks down once the metal particle volume filler fraction is too high. Once the volume fraction of filler is high enough the best way to describe the material properties is to use a theory that accounts for the inter-particle interaction such as that developed by McLachlan [ , ]. This theory has more complexity than that developed by Bruggeman and requires data measurements in order to determine some of the variables. The theory developed by McLachlan will not be discussed further, and percolation theory will be used instead $[$,$] . Percolation theory will not allow for the effective permittivity to be$ calculated directly, however it will allow for one to estimate the effect of adding certain amounts and types of particles to a composite. Furthermore, this theory can be used to determine at what volume filler fraction large changes in effective permittivity will occur.

\section{Bruggeman Theory}

Bruggeman's theory is a popular one due to its simplicity [ ]. It will not give extremely accurate results, but it is useful for gaining understanding of what to expect from heterogeneous mixtures. The Bruggeman theory makes a few assumptions:

- Particle fillers are small when compared to the wavelength of the electromagnetic field imposed upon the material. 
- Inclusions do not interact with each other electrically.

- All inclusions are statistically similar in size and shape.

- All particles are distributed randomly.

- The volume average polarizability is zero.

This theory breaks down when volume loadings come close to the percolation threshold. The percolation threshold is the point at which the volume filler fraction becomes high enough that inter-particle interaction cannot be ignored. Although there are many drawbacks to this theory it has one excellent virtue, its simplicity. The Bruggeman equation for spherical inclusions is:

$$
V F F \frac{\epsilon_{1}-\epsilon_{e f f}}{\epsilon_{1}+2 \epsilon_{e f f}}+(1-V F F) \frac{\epsilon_{2}-\epsilon_{e f f}}{\epsilon_{2}+2 \epsilon_{e f f}}=0
$$

where:

$V F F$ is the volume fraction of the conducting filler

$\epsilon_{1}$ is the permittivity of the filler material

$\epsilon_{2}$ is the permittivity of the matrix material

$\epsilon_{e f f}$ is the effective permittivity to be calculated using Equation (2.18)

\section{Percolation Theory}

Percolation theory is an important tool when analyzing disordered composite materials. Introduction to Percolation Theory [ ] gives a good description on percolation theory for any reader that is interested in having a greater understanding. Percolation theory is primarily concerned with what is known as the percolation threshold. 
Assume a square lattice representing a 2-D resistor network that is large enough that the boundary effects can be ignored. The edges of each square represent a node. The grid shown in Figure 2.7(a) shows a small section of the square lattice. Now each square is randomly filled with a resistor to each interface as shown in Figure 2.7(b) with a given probability. A cluster is defined as a group of touching filled squares. The clusters are circled in Figure 2.7(c).

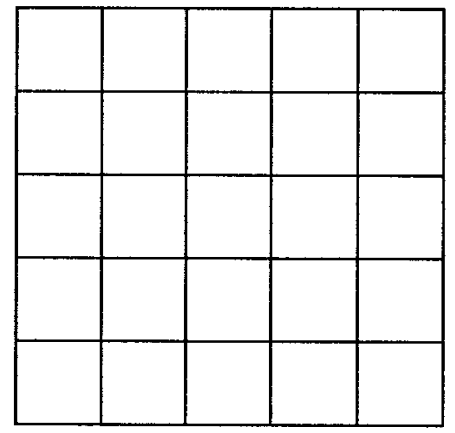

(a) Section of empty grid

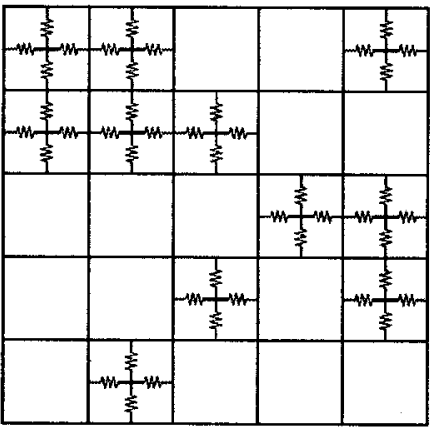

(b) Randomly filled grid

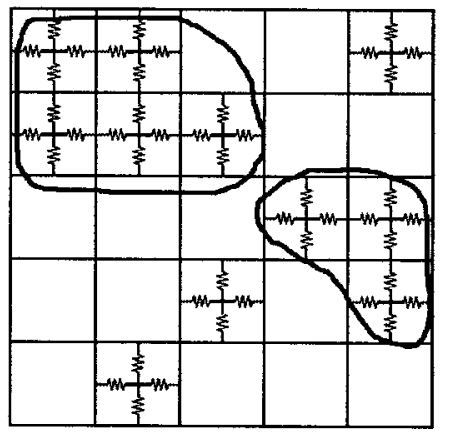

(c) Clusters circled

Figure 2.7: Section of 2-D resistor network

In Figure 2.7(c), the clusters circled are ones that the edges touch and are called 'nearest neighbor'. A cluster formed of edge and corner touching squares is called 'next nearest neighbor'. The percolation threshold is the fill probability at which one cluster forms a continuous network throughout the entire material as shown in Figure 2.8. This means that there will be a conduction path connecting all edges of the square lattice for the 2-D resistor network. 


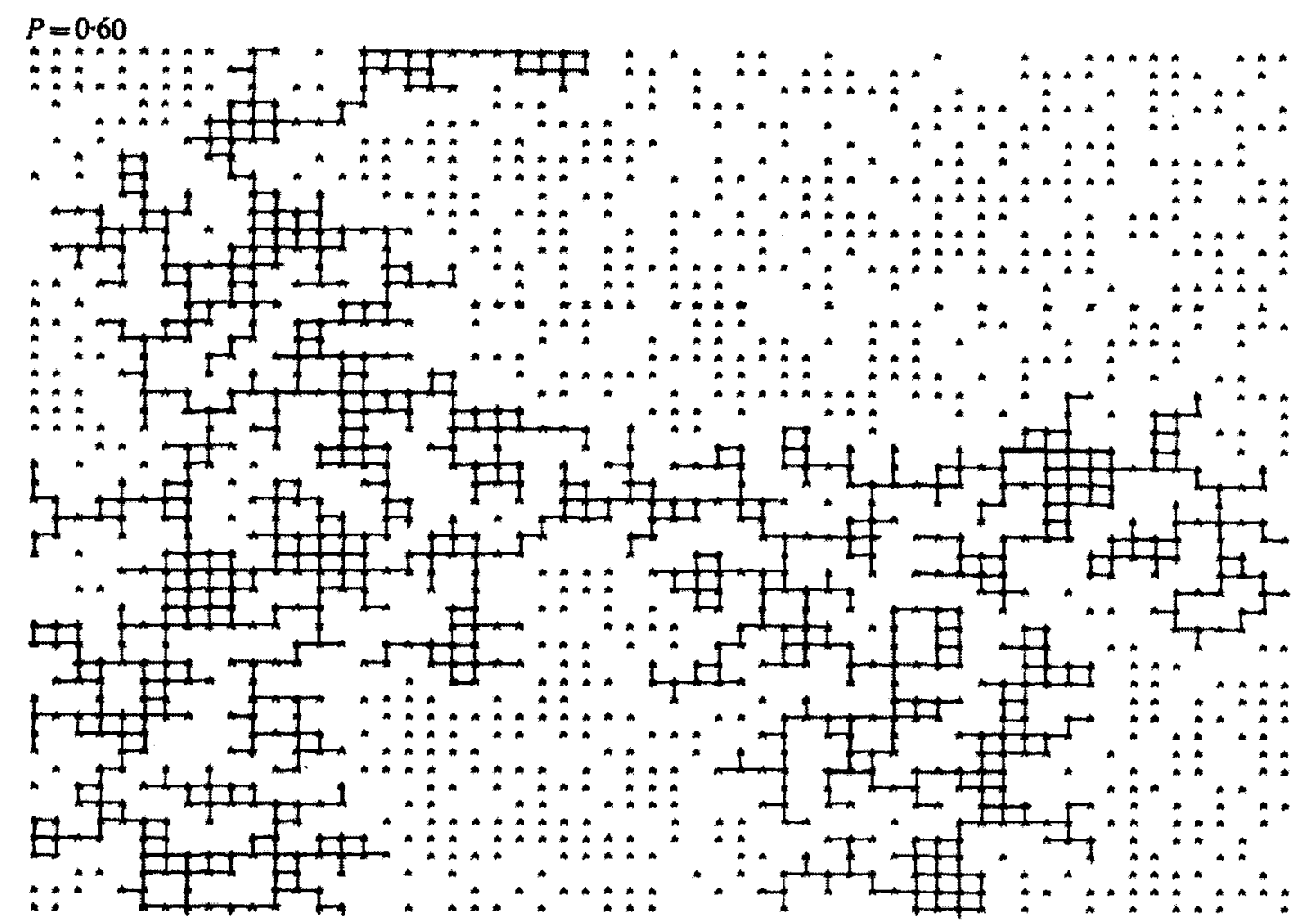

Figure 2.8: Example of percolation with a square lattice when each space is filled randomly with a probability of $60 \%$ [?]

If one randomly disperses a conducting filler particle in an insulating material some interesting material properties occur near the percolation threshold. Close to the percolation threshold, the real and imaginary part of the permittivity increase rapidly, making the percolation threshold an important point. Percolation theory is not going to give a precise solution as to what material properties one should expect. Rather it will give an idea of what volume filler fraction is necessary to achieve a rapid change in complex permittivity. There are many factors that can affect the percolation threshold. Two such factors are the size and shape of particles as shown below. Figures 2.9 and 2.10 below use particles made of a metal with conductivity of $10^{5} \mathrm{~S} / \mathrm{cm}$. The metal is coated with a shell that is $200 \mathrm{~nm}$ thick 
and has a conductivity of $10 \mathrm{~S} / \mathrm{cm}$. The particles are embedded in an insulator with a conductivity of $3 \times 10^{-17}$. Figure 2.9 suggests that particles with a large axial ratios will have a low percolation threshold. Figure 2.10 suggests that the smaller the particle, lower the percolation threshold. This means that small particles with a large axial ratio should have the lowest percolation threshold.

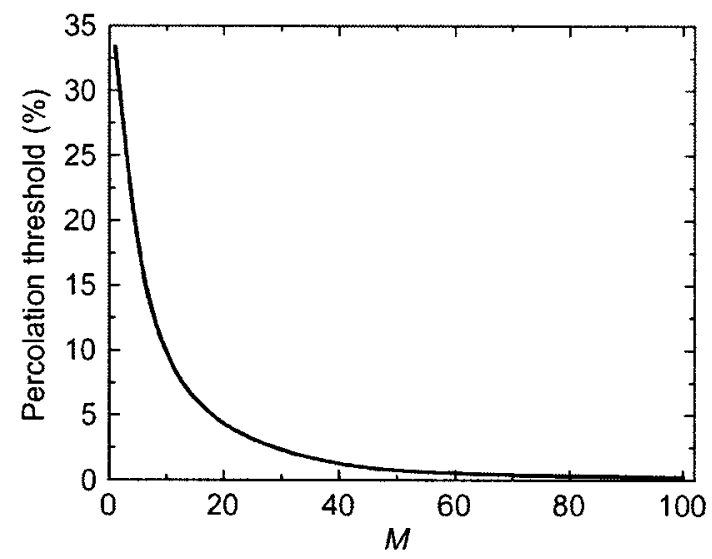

Figure 2.9: Effect of ellipsoidal axial ratio (M) of filler particle on percolation threshold. [1]

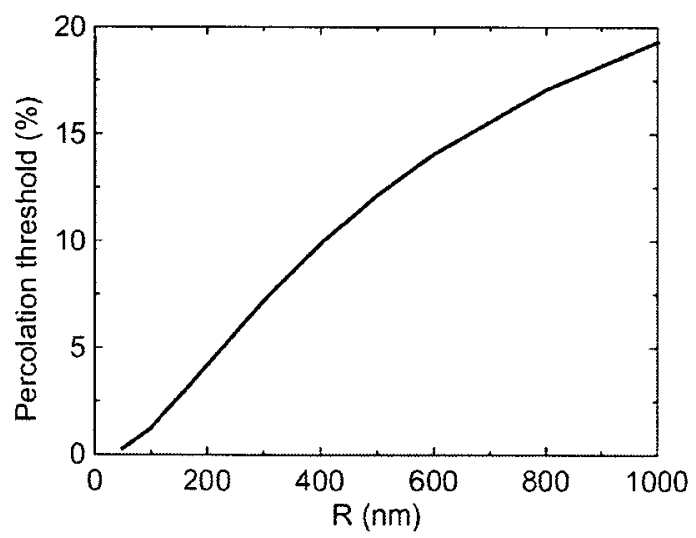

Figure 2.10: Effect of particle size ( $\mathrm{R}$ is radius) of filler particle on percolation threshold [ ] 


\subsection{Photoconduction}

Photoconduction is an effect whereby a material absorbs high energy electromagnetic waves (eg. light) and as a result the conductivity of the material increases. Some texts that cover photoconductivity are: Principles of Electronic Materials and Devices [ ] and Dielectric Phenomena in Solids [ ]. Unless otherwise referenced the material presented below is condensed from Principles of Electronic Materials and Devices [ ] The conductivity of a material is given by the following equation:

$$
\sigma=q\left(N_{e} \mu_{e}+N_{h} \mu_{h}\right)
$$

where:

$q$ is the charge of an Electron

$N_{e}$ is the number of electrons per unit volume

$N_{h}$ is the number of holes per unit volume

$\mu_{e}$ is the electron mobility

$\mu_{h}$ is the hole mobility

From the above equation one can see that there are two ways to change the conductivity of a material. The first way is to change the carrier mobility. However this is a property of the material which means the material itself must be changed. The other way is to change the concentration of charge carriers, which can be accomplished via illumination of some materials. The equation that gives the change in conductivity of a material upon illumination is:

$$
\Delta \sigma=\frac{q \eta I \lambda \tau_{r}\left(\mu_{e}+\mu_{h}\right)}{h c t}
$$

where: 
$I$ is the intensity of the light

$\eta$ is the quantum efficiency

$\lambda$ is the wavelength of the incident light

$\tau_{r}$ is the recombination time

$h$ is Plank's constant

$c$ is the speed of light

$t$ is the thickness of the material

Quantum efficiency is the number of electron-hole pairs generated per absorbed photon. The recombination time is the average lifetime of an electron-hole pair. Recombination time can be effected by the intensity of light, the temperature of the material, the size of particles, the number of crystal defects in the particles, and numerous other factors [.]. For the purposes of this work there is no need to discuss recombination further.

\subsection{Tunability}

Tunable microwave circuits have been realized by using materials with adjustable properties or by using a microwave switch to change the state of the circuit. These techniques are usually employed in one or more of the circuits that achieve tunability by: change of impedance; change of wave propagation properties of a structure; or change of the path the microwave signal follows.

Changing the impedance is usually achieved with lumped components such as tunable capacitors (varactors) and tunable inductors. Varactors can be made in three ways. The first way is using a semiconductor material and creating a device that has an electrically variable depletion area $[, ;]$. Another method is to use a ferroelectric 
material in a capacitor [ , ]. The last method is to use a microelectromechanical system (MEMS) to create a varactor [ ]. Tunable inductors can be made by using switches to change the configuration of the inductor [ ].

The wave propagation properties in a microwave structure can be dynamically changed by using materials with tunable permittivity or tunable permeability as the substrate or applying it as an overlay to the structure. Magnetically tunable filters [ ] and phase shifters [ ] have been realized by using magnetically tunable materials.

Switches are utilized in microwave circuits that do not require continuous tuning and can function acceptably with discrete tuning points. Switches are generally realized in one of the following three ways: transistor based switch; MEMS based switch [ ]; or photoconductive switch [.].

Optical control of semiconductor devices has been investigated and is reviewed in Optical Control of Microwave Semiconductor Devices [ ]. This paper discusses optical control of microwave amplifiers, oscillators, switches, and mixers. Optical control is achieved by generating charge carriers in the semiconductor material, or by an indirect means such as using a photodetector to covert the optical signal to an electrical signal. A paper entitled Tunable Micrwoave Load Based On Biased Photoinduced Plamsa in Silicon [ . ] discusses optically tuning a quarter-wave resonator by using a photoconductive semiconductor material. In this paper the resonant frequency of the quarter-wave resonator is shifted by $60 \%$ by illuminating the end of the resonator to change the effective capacitance and resistance of the resonator.

Ferroelectric materials are the most commonly used material reported in the literature that possess a tunable dielectric constant. Since this work proposes a new optically tunable dielectric material, the properties of ferroelectric materials are discussed further for the purpose of comparison. 


\subsubsection{Ferroelectric Materials}

Ferroelectric materials have considerable potential in tunable microwave circuits [ ]. All the ferroelectric materials discussed are used in the paraelectric state rather than the ferroelectric state. Much of the work has been done at low frequencies (below $1 \mathrm{GHz}$ ), however some work investigates the materials at microwave frequencies and it is that work that is discussed. Some ferroelectric materials that have been characterized are summarized in Table 2.1. The material summarized in Table 2.1 is not close to comprehensive, it is only a small selection of materials with reasonable performance that have been characterized at microwave frequencies.

Table 2.1: Comparison of ferroelectric materials

\begin{tabular}{|c|c|c|c|c|c|c|}
\hline Material & $\epsilon_{r}$ & Tunability & Tan $\delta$ & Range Tan $\delta$ & Frequency & Voltage \\
& $@ 0 \mathrm{~V}$ & $\%$ & $@ 0 \mathrm{~V}$ & & $\mathrm{GHz}$ & $\mathrm{MV} / \mathrm{m}$ \\
\hline $\mathrm{PbSrTiO}_{3}[]$ & 1575 & 35 & 0.1 & - & 10 & 4 \\
\hline $\mathrm{SrTiO}_{3}[]$ & 6000 & 85 & 0.19 & $0.11-0.19$ & 10 & 4 \\
\hline $\mathrm{BaSrTiO}_{3}[]$ & 1622 & 22 & 0.03 & - & 7.7 & 0.81 \\
\hline
\end{tabular}

From the data presented above it can be seen that some of the materials have good characteristics. However, it does not give a good idea of how these materials respond over a frequency range or at different bias fields. The typical dependence of a ferroelectric material on frequency and bias field is shown in Figure 2.11. 

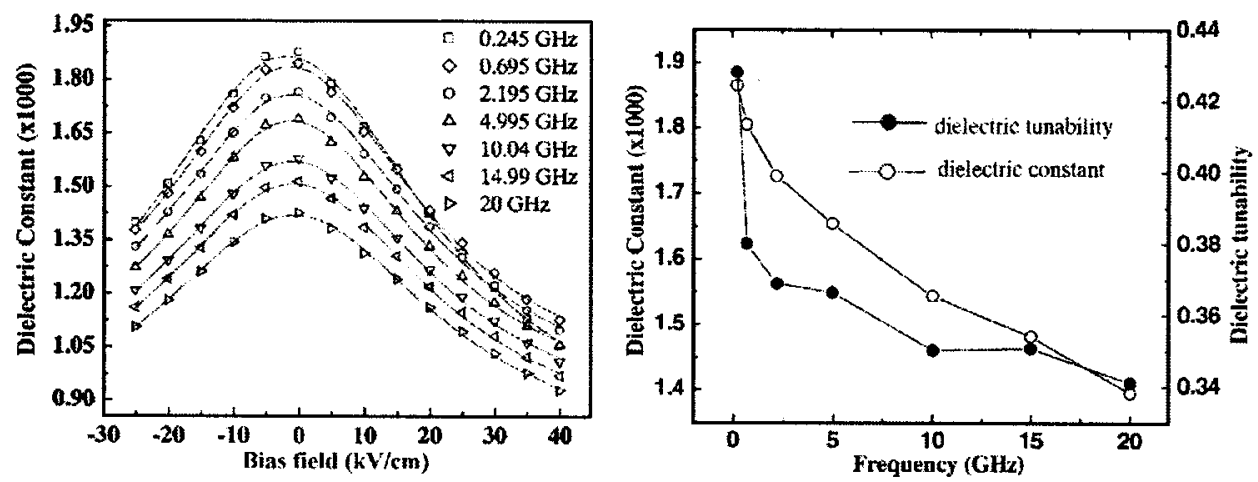

(a) Frequency and bias depencence of di- (b) Frequency dependence of zero-bias dielectric constant for $\mathrm{Pb}_{0} \cdot 35 \mathrm{Sr}_{0} \cdot 65 \mathrm{TiO}_{3}$ electric constant and dielectric tunability of $\mathrm{Pb}_{0} .35 \mathrm{Sr}_{0} .65 \mathrm{TiO}_{3}$

Figure 2.11: Typical response of ferroelectric materials [ ]

The dielectric constant and dielectric loss tangent of another material is shown in Figure 2.12.
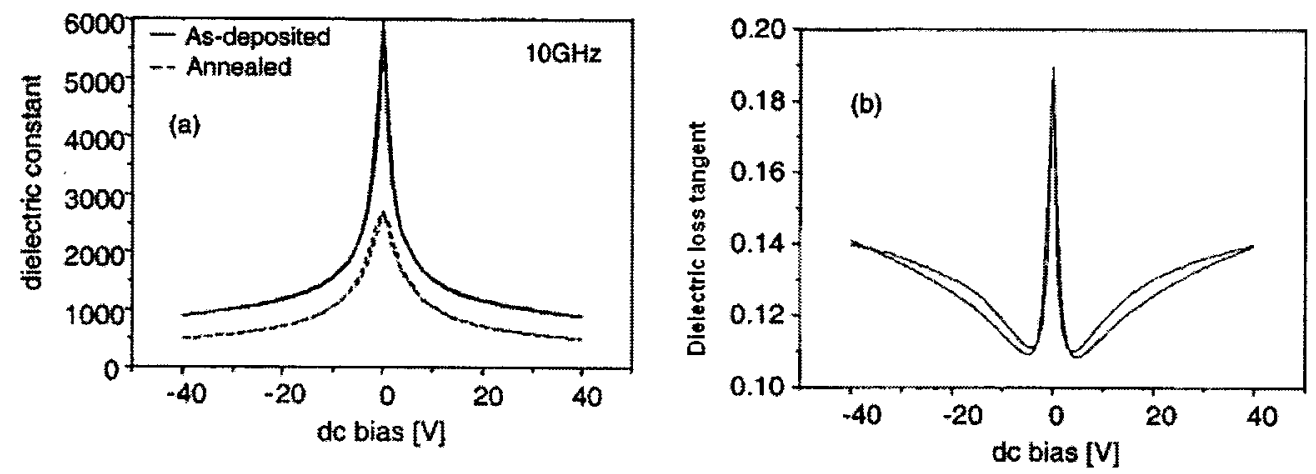

(a) Dependence of dielectric constant on bias (b) Dependence of dielectric loss tangent on field bias field

Figure 2.12: Properties of $\mathrm{SrTiO}_{3}$ at $10 \mathrm{GHz}$ []

From Figures 2.11 and 2.12 it can be seen that the dielectric constant and the dielectric loss tangent are highest with zero bias, which is typical behaviour of ferroelectric materials in the paraelectric phase. The dielectric constant of most of these 
materials decreases with frequency, while the dielectric loss tangent increases with frequency. Also, the tunability of the ferroelectric materials usually decreases with frequency. There is relatively little data on the temperature stability of ferroelectric materials at microwave frequencies, however data taken at $1 \mathrm{MHz}$ as shown in Figure 2.13 can at least provide an approximation.

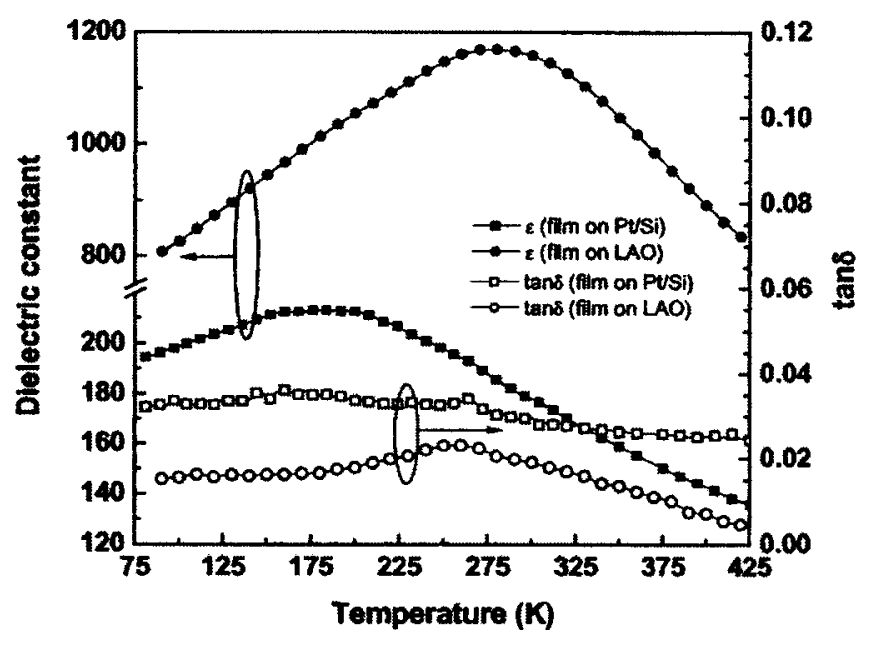

Figure 2.13: Dielectric constant and loss tangent of $\mathrm{Pb}_{0} .3 \mathrm{Sr}_{0} .7 \mathrm{TiO}_{3}$ films at $1 \mathrm{MHz}$ []

When a ferroelectric material is used in a capacitor, the properties of the capacitor can change drastically with temperature as shown in Figure 2.14. Once the temperature becomes low enough the material is in the ferroelectric state and the effects of the hysteresis loop can be seen.
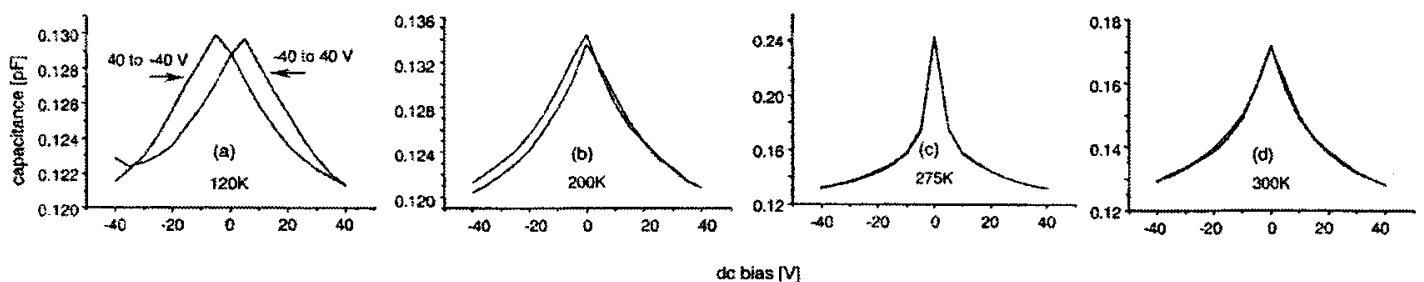

Figure 2.14: Temperature and bias dependence of ferroelectric capacitors at $10 \mathrm{GHz}$ (a) $120 \mathrm{~K}$ (b) $200 \mathrm{~K}$ (c) $275 \mathrm{~K}$ (d) $300 \mathrm{~K}$ [ ] 


\subsection{Summary}

This chapter began by discussing the complex permittivity of materials. There are many different properties that affect the permittivity of materials, and these properties are called polarization. There are five types of polarization: electronic, atomic, orientational, space charge, and spontaneous. Electronic polarization is associated with the displacement of electrons with an applied electric field. Atomic polarization deals with the displacement of atoms with an applied electric field. Orientational polarization is the movement of polar molecules when an electric field is applied. Spontaneous polarization only occurs in a special class of materials known as ferroelectric materials. These materials show a large change in permittivity with an applied electric field. Space charge polarization is the movement of free charge carriers with an applied electric field. Space charge polarization is of importance in this work because it describes what is happening when metallic particles are added to a non-conductive material.

Next, effective medium theories and percolation theory were discussed. Effective medium theories describe the dielectric properties of heterogeneous mixtures, usually consisting of a non-conductive host with conductive constituents. The addition of metallic particles in a non-conductive host increases the effective dielectric constant and dielectric loss tangent of the material. Most effective medium theories do not account for inter-particle interactions and break down once the volume fill fraction of metallic particles exceeds a certain percentage. At this point percolation theory is used to describe the material. Percolation theory identifies that volume fill fraction of metallic particles at which the effective electrical material properties change drastically.

Next, the effect known as photoconduction was presented. Photoconduction is an 
effect whereby the conductivity of a material increases when illuminated with high energy electromagnetic waves (optical frequencies).

Finally, the chapter is concluded with a discussion on tunable circuits. Circuits that are tunable using mechanical, electrical, and magnetic methods have been made. Electrically tunable circuits have been created using ferroelectric materials in the paraelectric state. Overall ferroelectric materials have potential in tunable microwave circuits. They demonstrate appreciable tunability, low enough dielectric loss tangent, and the need for high bias fields can be overcome by using the material in varactors. However, the issue of temperature stability may cause issues for their use in some applications. 


\section{Chapter 3}

\section{Optically Tunable Dielectric Material}

\section{Analysis}

\subsection{Introduction}

Ferroelectric materials have some drawbacks for use as tunable dielectric materials. The effect that ferroelectric materials utilize is heavily temperature dependant. Ferroelectric materials need to be crystalline in structure which makes them more expensive and difficult to use. Since ferroelectric materials are tuned electrically, there are limitations on the field strength of the microwave signals and where and how they can be used.

A new optically tunable dielectric material is proposed. This material uses a composite consisting of photoconductive spheres dispersed in an insulating polymer. Using effective medium theory discussed earlier it can be seen that changing the conductivity of the spheres can change the effective permittivity of the material. This new material does not suffer from many of the problems that affect the performance of ferroelectric materials. Unlike ferroelectric materials this method does not depend upon an effect that is heavily temperature dependant. Changes in temperature will 
have an effect on the photoconductivity of the spheres, however photoconductivity is not strongly sensitive to changes in temperature. Since the tuning of the material is done optically it means that there is an inherent isolation between the tuning signal and the microwave signal. The processing involved for this material is simplistic by comparison to the processing needed for ferroelectric materials, making it a cheaper alternative.

This chapter demonstrates the effects of adding conductive particles to a nonconductive host material, and is done using theoretical information and simulations. The following section uses properties of materials and effective medium theory to demonstrate the effect of adding a photoconductive material to a non-conductive host material. In the next section, simulations run using a numerical electromagnetic solver are compared to theoretical results.

\subsection{Theoretical Observations}

This section begins by discussing the properties of CdS and the level of conductivity expected given a specific level of optical illumination. Next, Bruggeman's effective medium theory is used to show the effects of mixing conductive particles with a non-conductive matrix. After showing material properties using Bruggeman's theory, percolation theory is used to demonstrate the effect of large volume fill fractions of the photoconductive particle.

CdS is used because it has been studied previously at Carleton University and shows promise for optical tuning [ ]. The particles of CdS that will be employed are approximated as spherical and have an average diameter of $1 \mu \mathrm{m}$.

The properties of bulk CdS are as follows:

$$
\mu_{n}=160 \mathrm{~cm}^{2} / V s[\text { ] }
$$




$$
\begin{aligned}
& \mu_{h}=15 \mathrm{~cm}^{2} / V s[] \\
& \epsilon(0)=8.5[] \\
& \epsilon(\infty)=5.26[]
\end{aligned}
$$

$\tau$ is $22.3 \mu \mathrm{s}$ to $57.4 \mu \mathrm{s}$ [ ]

Bandgap is $2.4 \mathrm{eV}[\mathrm{]}$

The bandgap of CdS is $2.4 \mathrm{eV}$, which means that wavelengths shorter than $520 \mathrm{~nm}$ will be absorbed and create a single electron-hole pair. There are a number of assumptions that will be made when calculating the photoconductivity of the material. These assumptions are: the material is uniformly illuminated; the minority carrier lifetime of the material is not affected by strong illumination; all spheres dispersed in the material get illuminated equally or the charge diffuses to them all equally. The last assumptions are: the power of the light source is $0.0002171 \mathrm{~W} / \mathrm{mm}^{2}$; the minority carrier lifetime is $22.3 \mu s$; and the volume fill fraction of photoconductive particles is $72 \%$. Then the change in conductivity upon illumination can be calculated using the Equation (3.1).

$$
\begin{gathered}
\Delta \sigma=\frac{q \eta I \lambda \tau\left(\mu_{e}+\mu_{h}\right)}{h c t V F F} \\
\Delta \sigma=9.85 S / m
\end{gathered}
$$

This shows that a conductivity of approximately $10 \mathrm{~S} / \mathrm{m}$ can be expected and it should be possible to achieve conductivities of $100 \mathrm{~S} / \mathrm{m}$ by using a different material or a higher intensity light source. The conductivity range of $0.01 \mathrm{~S} / \mathrm{m}$ to $100 \mathrm{~S} / \mathrm{m}$ will be used for calculations and simulations. It will be assumed that the polymer used as a host for the CdS has a dielectric constant of 3 and a dielectric loss tangent of 
0.001. Using Bruggeman's theory the effects of the real and imaginary parts of the permittivity for the filler and host can be investigated. The default values for the filler material are a dielectric constant of 8.5 and a conductivity of $10 S / m$, while those for the host material are a dielectric constant of 3 and a dielectric loss tangent of 0.001 . Furthermore, if the volume fill fraction is not specified it is $10 \%$. All the following results are for a frequency of $8 \mathrm{GHz}$. In order to see the effects of each parameter, they are swept independently of the other values and the results are shown in Figures $3.1-3.3$.

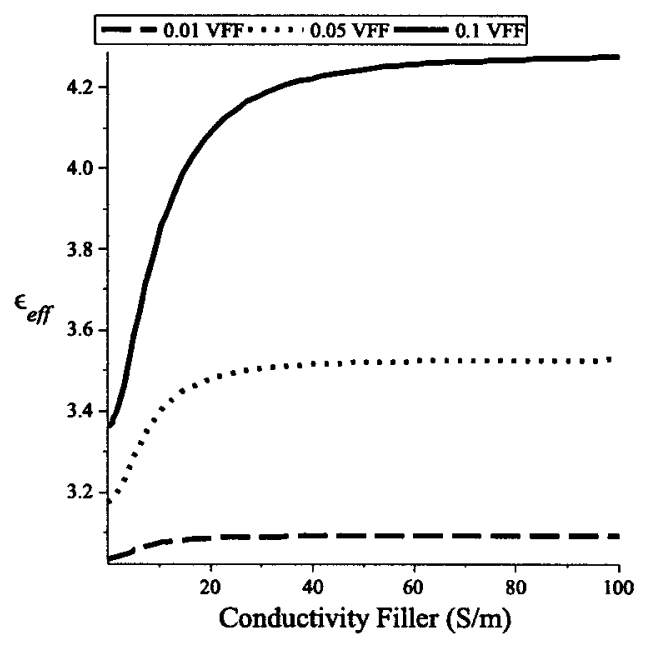

(a) Dielectric constant

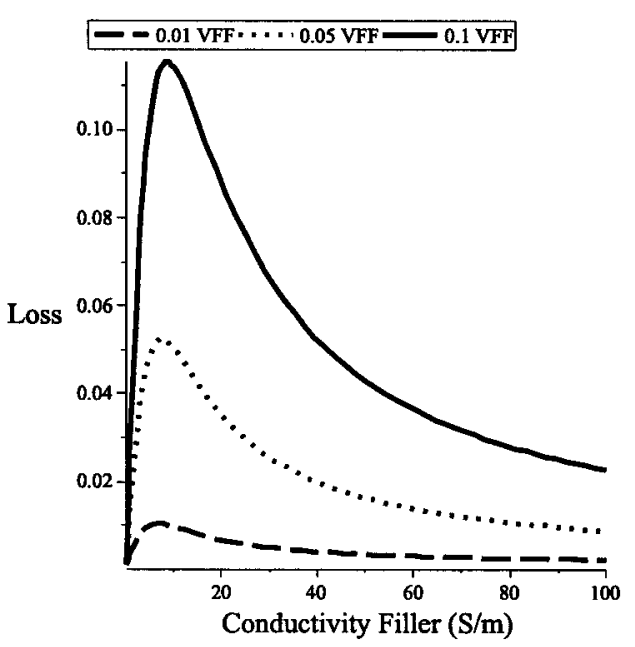

(b) Dielectric loss tangent

Figure 3.1: Effect of the conductivity of the filler on $\epsilon_{\text {eff }}^{\prime}$ and dielectric loss tangent 


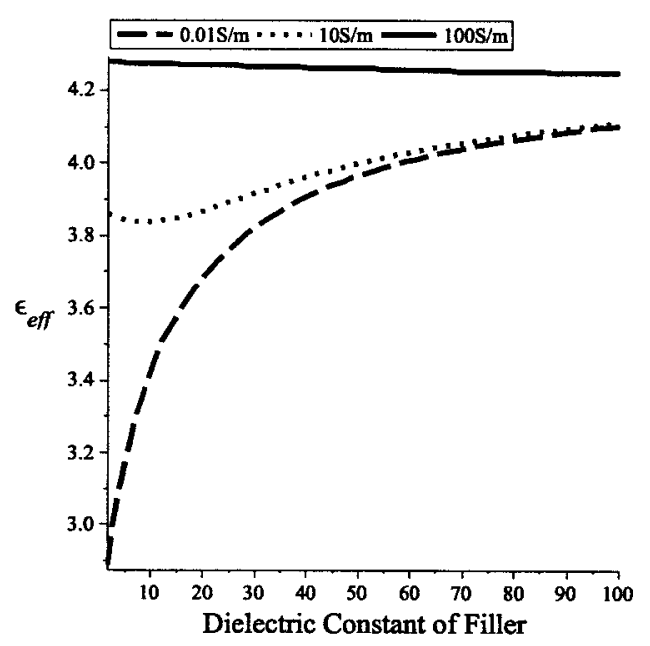

(a) Dielectric constant

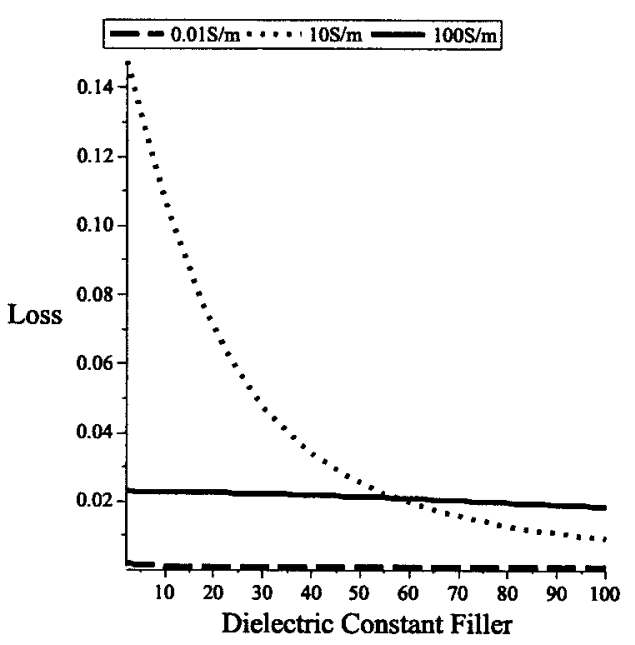

(b) Dielectric loss tangent

Figure 3.2: Effect of the dielectric constant of the filler on $\epsilon_{e f f}^{\prime}$ and dielectric loss tangent

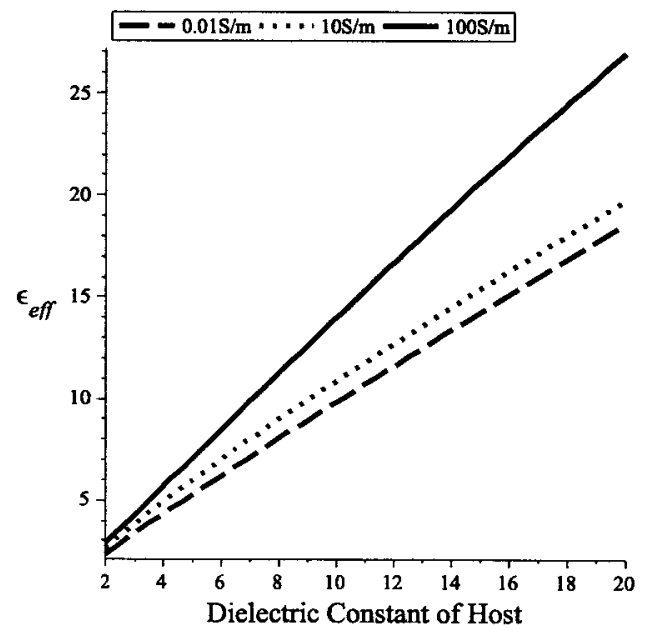

(a) Dielectric constant

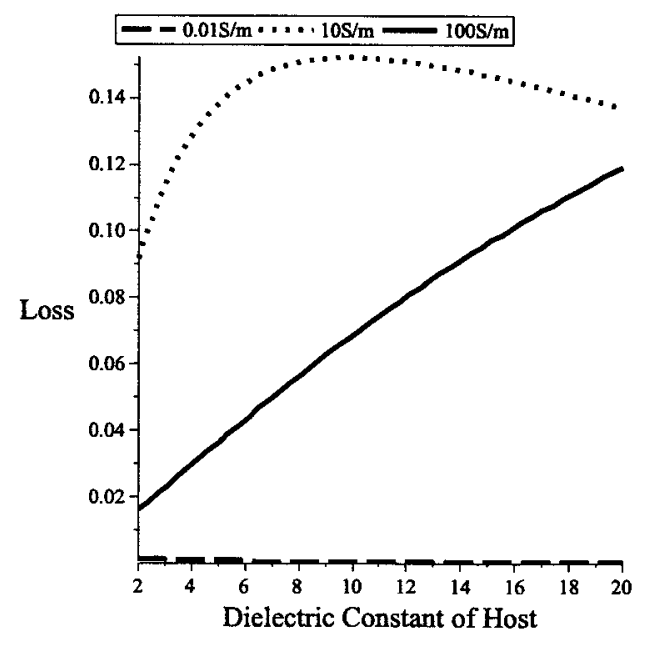

(b) Dielectric loss tangent

Figure 3.3: Effect of the dielectric constant of the host on $\epsilon_{\text {eff }}^{\prime}$ and dielectric loss tangent

Bruggeman's theory does not account well for large volume fill fractions of metallic inclusions, and this is where percolation theory is useful. Percolation theory predicts 
that as the volume fill fraction of the filler particle comes close to the percolation threshold, there will be a large change in both the real and imaginary parts of the permittivity. The next section demonstrates that simulations can be used to predict properties of composite materials.

\subsection{Numerical Analysis}

This section demonstrates that a numerical electromagnetic solver (HFSS in this case) can be used to predict material properties. Using computational electromagnetics to predict the properties of composite materials is not new [ ]. This section is presented to familiarize the reader with a potential approach for developing optically tunable materials. This is done by performing simulations of composite materials and comparing them to theoretical models. Simulations are run to compare to Bruggeman's theory, and to demonstrate the simulations can predict the percolation threshold.

The free space setup discussed in Section 4.2 was constructed in HFSS and a representation is shown in Figure 3.4. The setup consisted of a long rectangular prism (10 $\mu \mathrm{m} \times 10 \mu \mathrm{m}$ and $6 \mathrm{~mm}$ in length) filled with vacuum, and the MUT was in the center (10 $\mu \mathrm{m}$ thick). Two sides have a perfect electric conductor and two sides have a perfect magnetic conductor assigned as the boundary conditions. The electric field was excited so that it was normal to the perfect electric conductors and tangential to the perfect magnetic conductors. The boundary conditions and excitation allowed for a plane wave incident upon a laterally infinite material to be simulated. To confirm this setup functioned as expected, simulations with the MUT having different electric and magnetic properties were run. Then the theory described in Section 4.2 was used to extract the properties of the material simulated by using the s-parameters. The 
electric and magnetic properties of the material extracted matched the properties of the material simulated, thus confirming the simulation could be relied upon.

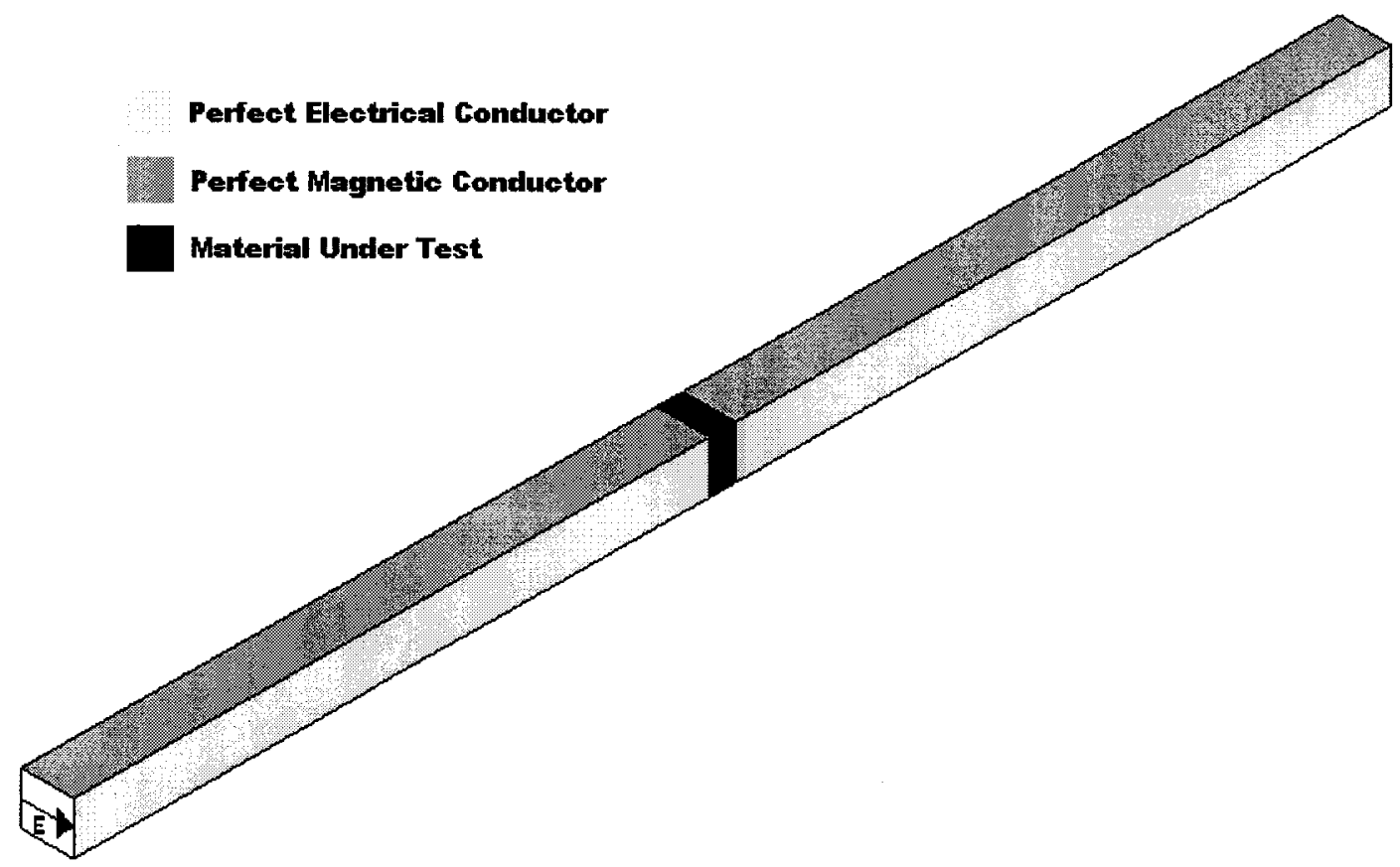

Figure 3.4: Setup used to simulate heterogeneous materials (Top and bottom has perfect magnetic boundary condition, while sides have perfect electric conductor boundary condition)

The next step was to simulate a heterogeneous material. In order to compare this material to Bruggeman's theory a material with spheres dispersed in it was simulated. Unfortunately, simulating spheres in HFSS was impractical. This was due to the fact that meshing a sphere in a rectangular coordinate system required too many elements. For this reason the sphere was approximated as a cube with a square frustum on each face as shown in Figure 3.5. The cube was made to fit within a sphere with a diameter of $1 \mu \mathrm{m}$. The approximation of the sphere shown in Figure 3.5 was aligned so that the top of each frustum would be in contact with any of the adjacent sphere approximations. 


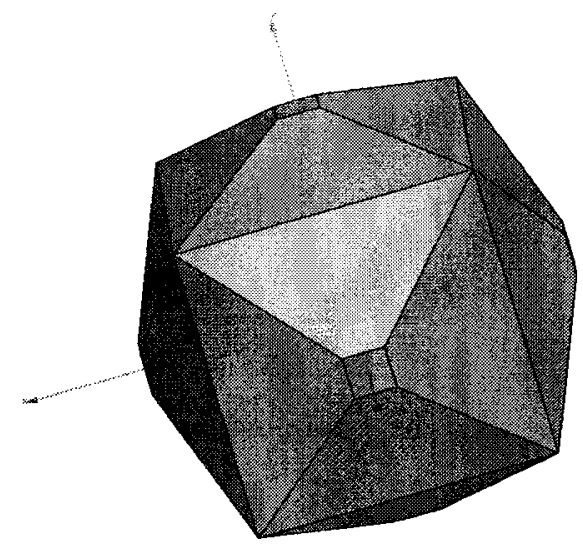

Figure 3.5: Shape used to approximate a sphere

The heterogeneous material was represented by the $10 \mu \mathrm{m}$ cube broken into smaller $1 \mu \mathrm{m}$ cubes. Each cube was then randomly (the random function is Matlab was used) filled with the shape given in Figure 3.5 to give the desired volume fill fraction. A material filled with a volume fill fraction of $5 \%$ is shown in Figure 3.6.

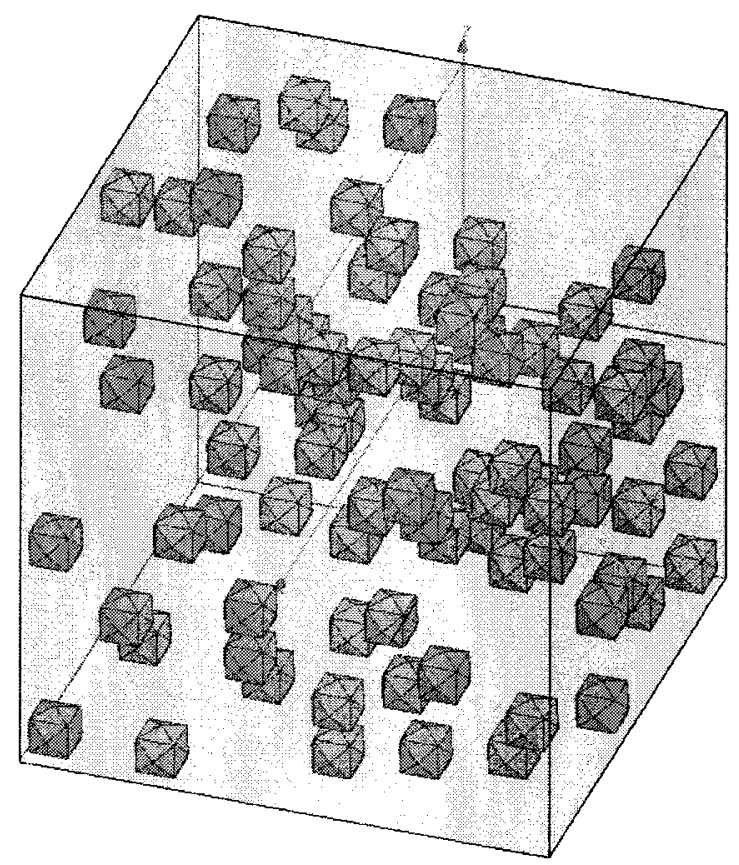

Figure 3.6: Material with a $5 \%$ volume fill fraction 
This method of representing the material had a few potential problems. Most of these problems related to decreased accuracy of the simulation results. Firstly, the small size of the sample being simulated may lead to variations in the permittivity of the material extracted. For this reason, three separate simulations were run for each configuration. This decreases the chance of getting erroneous results by confirming that the specific arrangement of particles was not causing the results.

All of the following simulations and data are simulated or calculated at $8 \mathrm{GHz}$.

The first set of simulations ran was to confirm that simulation results matched what Bruggeman's theory predicts. The results from simulating the effects of the conductivity and volume fill fraction of the filler are shown in Table 3.1. The data presented in Table 3.1 had two subsets of simulations performed; each simulation used different surface areas for the top of the square frustum. Setup 1 used a frustum with the top having a surface area of $0.01 \mu \mathrm{m}^{2}$, while setup 2 had a surface area of $0.0001 \mu \mathrm{m}^{2}$. The results from setup 2 are compared graphically to Bruggeman's theory in Figures 3.7 and 3.8. The effects of the dielectric constant and the conductivity of the filler are shown in Table 3.2 and in Figures 3.9 and 3.10. The effects of the dielectric constant of the host and conductivity of the filler are shown in Table 3.3 and in Figures 3.11 and 3.12. The simulation performed to get the data shown in Tables 3.3 and 3.2 used square frustums with a top surface area of $0.0001 \mu \mathrm{m}^{2}$. 
Table 3.1: Effect of conductivity and volume fill fraction of the filler on $\epsilon_{\text {eff }}^{\prime}$ and dielectric loss tangent (The properties of the host matrix are $\epsilon_{r}^{\prime}=3$, tan $\delta=$ 0.001 )

\begin{tabular}{|c|c|c|c|c|c|c|c|}
\hline VFF $(\%)$ & Conductivity (S/m) & $S_{e} t u p l \cdot \varepsilon_{e f f}$ & Setupl $I$ tand & $S_{i} / 14=2, E_{t f}$ & Sermpz $\tan \delta$ & Bruggemam. $\epsilon_{\text {of }}$ & Brmggemom, tand \\
\hline 1 & 0.001 & 3.040 & 0.001 & 3.038 & 0.001 & 3.034 & 0.001 \\
\hline 1 & 1.000 & 3.041 & 0.004 & 3.039 & 0.004 & 3.036 & 0.004 \\
\hline 1 & 10.000 & 3.086 & 0.014 & 3.082 & 0.013 & 3.075 & 0.010 \\
\hline 1 & 100.000 & 3.132 & 0.007 & 3.117 & 0.005 & 3.093 & 0.002 \\
\hline 5 & 0.001 & 3.185 & 0.001 & 3.176 & 0.001 & 3.176 & 0.001 \\
\hline 5 & 1.000 & 3.191 & 0.016 & 3.182 & 0.016 & 3.183 & 0.015 \\
\hline 5 & 10.000 & 3.408 & 0.061 & 3.392 & 0.056 & 3.396 & 0.050 \\
\hline 5 & 100.000 & 3.678 & 0.038 & 3.568 & 0.026 & 3.527 & 0.009 \\
\hline 10 & 0.001 & 3.372 & 0.001 & 3.355 & 0.001 & 3.365 & 0.001 \\
\hline 10 & 1 & 3.384 & 0.031 & 3.367 & 0.029 & 3.377 & 0.030 \\
\hline 10 & 10 & 3.822 & 0.119 & 3.796 & 0.107 & 3.837 & 0.114 \\
\hline 10 & 100 & 4.499 & 0.109 & 4.203 & 0.068 & 4.275 & 0.023 \\
\hline
\end{tabular}

Table 3.2: Effect of $\epsilon_{r}^{\prime}$ and conductivity of the filler on $\epsilon_{e f f}^{\prime}$ and dielectric loss tangent (The properties of the host matrix are $\epsilon_{r}^{\prime}=3, \tan \delta=0.001$ )

\begin{tabular}{|c|c|c|c|c|c|}
\hline Conductivity (S/m) & $\epsilon_{r}^{\prime} F i l l$ & $\epsilon_{\text {eff }}^{\prime}$ & $\tan \delta$ & Bruggeman, $\epsilon_{\text {eff }}{ }^{\prime}$ & Bruggeman, tan $\delta$ \\
\hline 0.01 & 2 & 2.90 & 0.0018 & 2.89 & 0.0019 \\
\hline 0.01 & 52 & 3.95 & 0.0010 & 3.98 & 0.0010 \\
\hline 0.01 & 100 & 4.10 & 0.0010 & 4.10 & 0.0010 \\
\hline 10 & 2 & 3.82 & 0.1350 & 3.86 & 0.1475 \\
\hline 10 & 52 & 4.01 & 0.0295 & 4.01 & 0.0242 \\
\hline 10 & 100 & 4.11 & 0.0132 & 4.11 & 0.0092 \\
\hline 100 & 2 & 4.20 & 0.0690 & 4.28 & 0.0229 \\
\hline 100 & 52 & 4.22 & 0.0606 & 4.26 & 0.0213 \\
\hline 100 & 100 & 4.25 & 0.0554 & 4.25 & 0.0185 \\
\hline
\end{tabular}

Table 3.3: Effect of dielectric constant of host and conductivity of filler on $\epsilon_{e f f}^{\prime}$ and dielectric loss tangent (The properties of the host matrix are $\epsilon_{r}^{\prime}=3, \tan \delta=$ $0.001)$

\begin{tabular}{|c|c|c|c|c|c|}
\hline Conductivity $(\mathrm{S} / \mathrm{m})$ & $\epsilon_{r}^{\prime}$ Host & $\epsilon_{\text {eff }}{ }^{\prime}$ & $\tan \delta$ & Bruggeman, $\epsilon_{\text {eff }}{ }^{\prime}$ & Briggeman, tan $\delta$ \\
\hline 0.01 & 2 & 2.34 & 0.0012 & 2.35 & 0.0020 \\
\hline 0.01 & 12 & 11.64 & 0.0011 & 11.62 & 0.0005 \\
\hline 0.01 & 20 & 18.67 & 0.0011 & 18.6 & 0.0003 \\
\hline 10 & 2 & 2.61 & 0.0901 & 2.66 & 0.0916 \\
\hline 10 & 12 & 12.71 & 0.1478 & 12.71 & 0.1509 \\
\hline 10 & 20 & 19.77 & 0.1344 & 19.77 & 0.1373 \\
\hline 100 & 2 & 2.88 & 0.0849 & 2.85 & 0.0161 \\
\hline 100 & 12 & 16.22 & 0.0805 & 16.7 & 0.0804 \\
\hline 100 & 20 & 26.38 & 0.1084 & 26.94 & 0.1194 \\
\hline
\end{tabular}




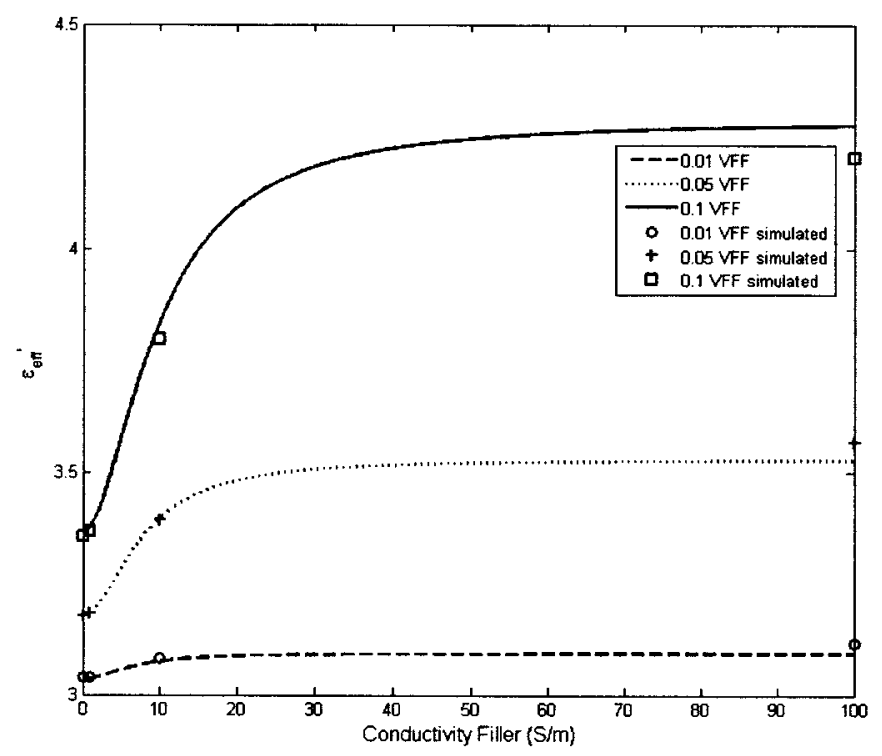

Figure 3.7: Effect of conductivity and volume fill fraction of filler on $\epsilon_{\text {eff }}^{\prime}$ (The properties of the host matrix are $\epsilon_{r}^{\prime}=3, \tan \delta=0.001$ )

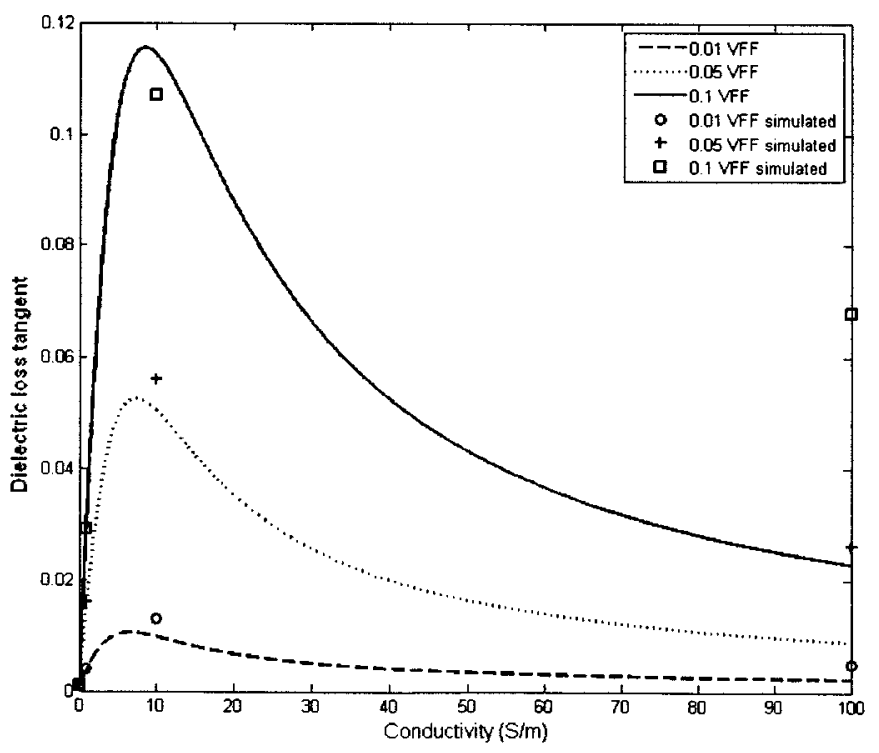

Figure 3.8: Effect of conductivity and volume fill fraction of filler on dielectric loss tangent (The properties of the host matrix are $\epsilon_{r}^{\prime}=3$, tan $\delta=0.001$ ) 


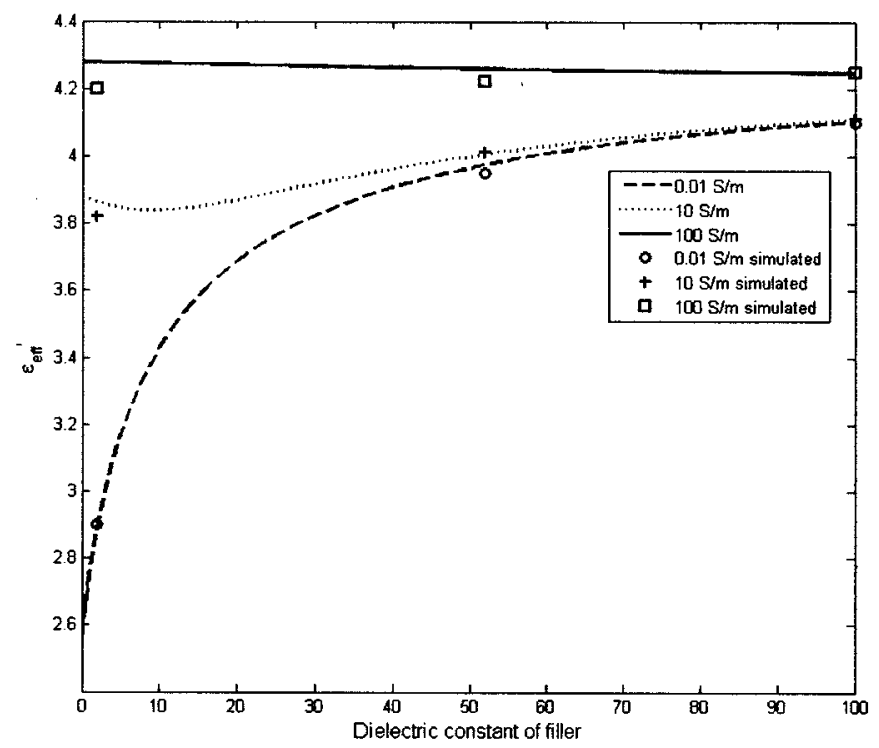

Figure 3.9: Effect of $\epsilon_{r}^{\prime}$ and conductivity filler on $\epsilon_{\text {eff }}^{\prime}$ (The properties of the host matrix are $\epsilon_{r}^{\prime}=3, \tan \delta=0.001$ )

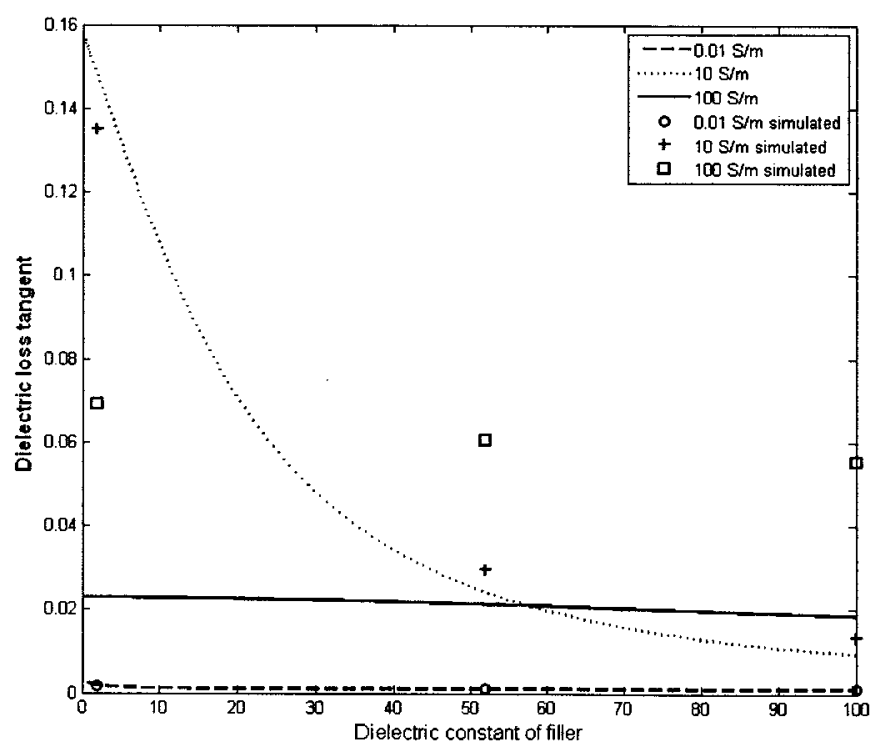

Figure 3.10: Effect of $\epsilon_{r}^{\prime}$ and conductivity filler on dielectric loss tangent (The properties of the host matrix are $\epsilon_{r}^{\prime}=3, \tan \delta=0.001$ ) 


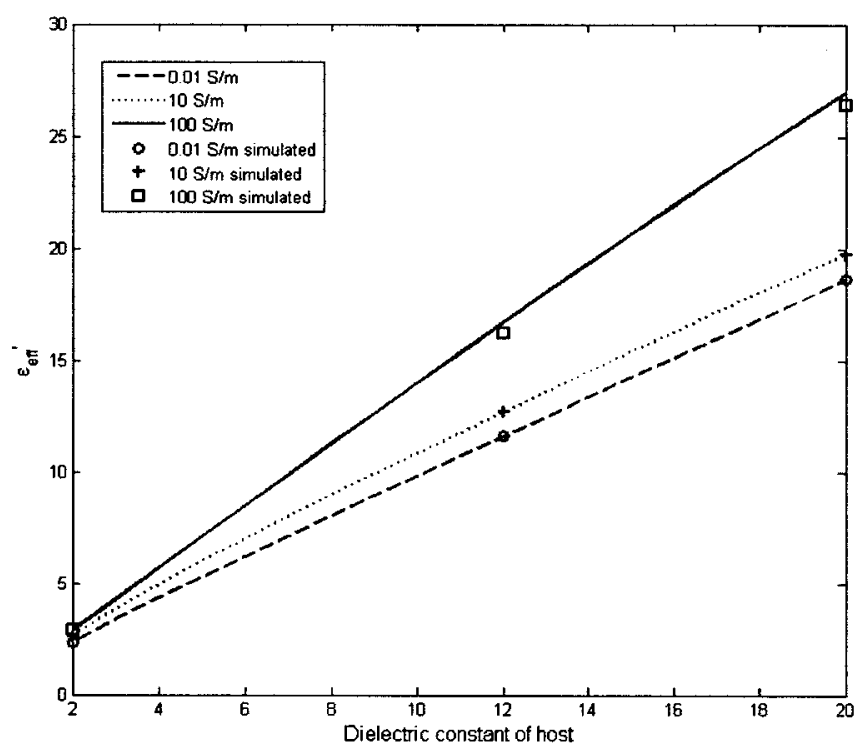

Figure 3.11: Effect of dielectric constant of host and conductivity of filler on $\epsilon_{\text {eff }}^{\prime}$ (The properties of the host matrix are $\epsilon_{r}^{\prime}=3, \tan \delta=0.001$ )

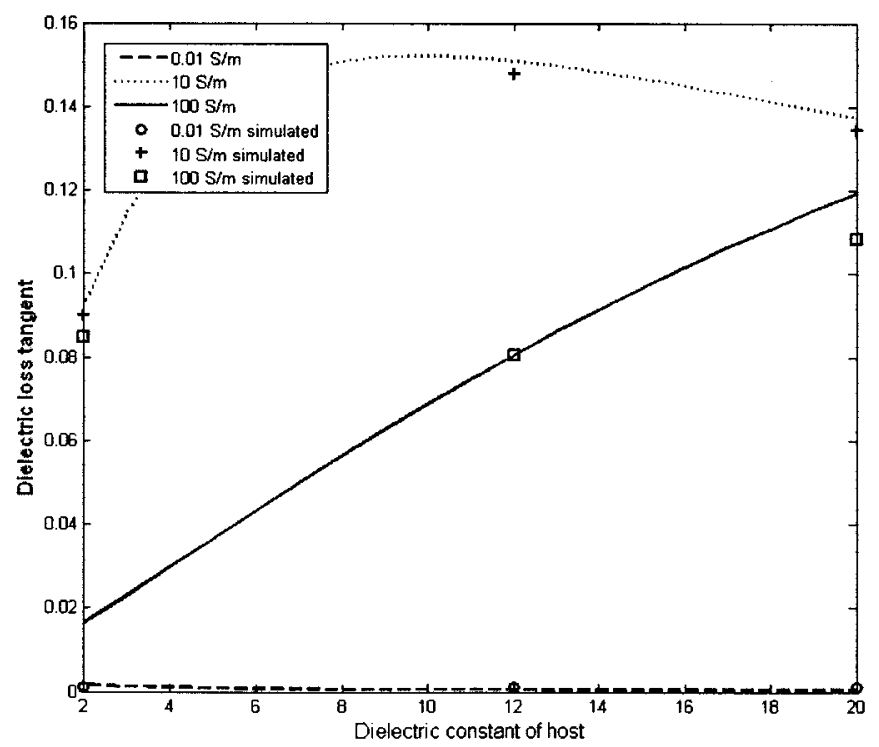

Figure 3.12: Effect of dielectric constant of host and conductivity of filler on dielectric loss tangent (The properties of the host matrix are $\epsilon_{r}^{\prime}=3, \tan \delta=0.001$ ) 
From the results presented in the above tables one can see that the results from simulation match rather closely with what Bruggeman's theory predicts. The data presented in Table 3.1 shows that as contact between filler particles decreases the dielectric loss tangent and effective dielectric constant also decreases. This result makes sense because this polarization is caused by the movement of charge. Differences in dielectric loss tangent can be attributed to the fact Bruggeman's theory uses spheres with much less contact area than the shapes used in simulations.

The next set of simulations was to confirm the simulations yielded the results predicted by percolation theory. The effect of volume fill fraction and conductivity on $\epsilon_{\text {eff }}^{\prime}$ and dielectric loss tangent is shown in Figures 3.13-3.16. The simulations use the same filler particle and configuration as the previous set of simulations. There are three points for each volume fill fraction because each point represents a different material, with a unique distribution of particles.

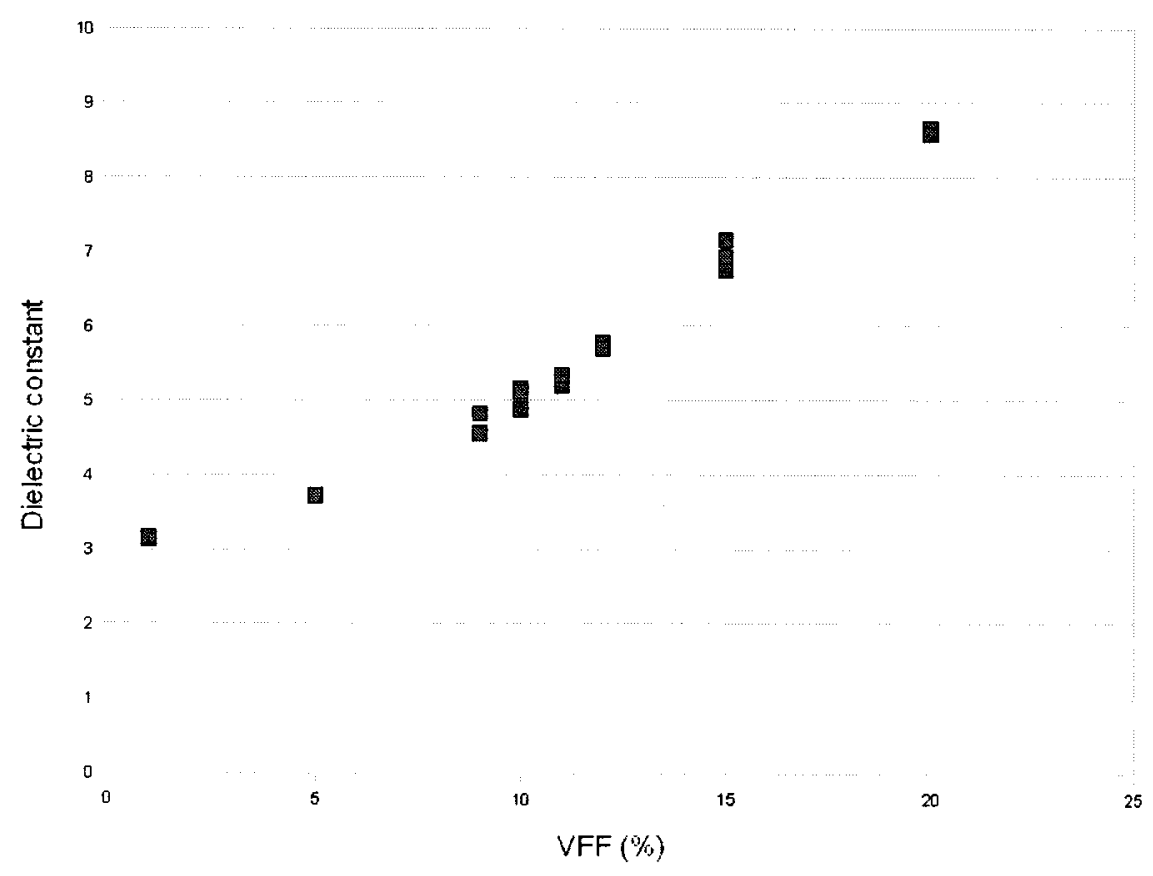

Figure 3.13: Dependence of $\epsilon_{\text {eff }}^{\prime}$ on volume filler fraction (filler particles have a conductivity of $1000 \mathrm{~S} / \mathrm{m}$ ) 


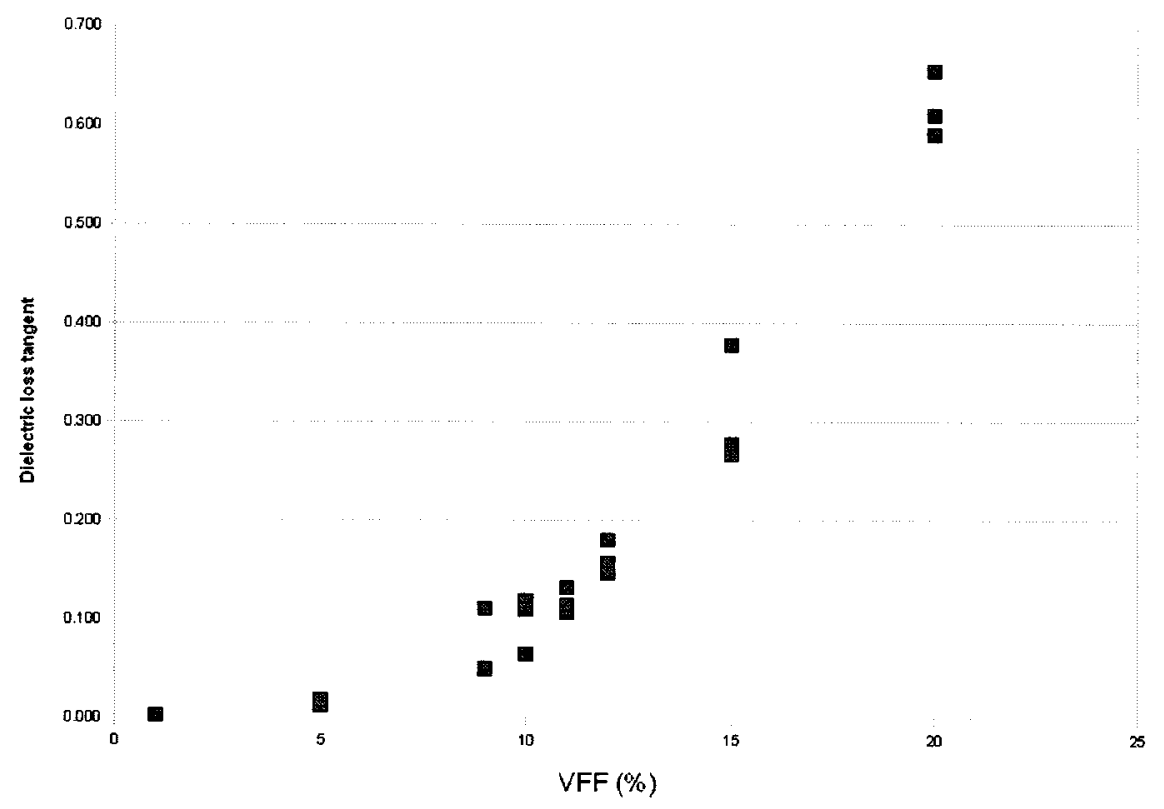

Figure 3.14: Dependence of dielectric loss tangent on volume filler fraction (filler particles have a conductivity of $1000 \mathrm{~S} / \mathrm{m}$ )

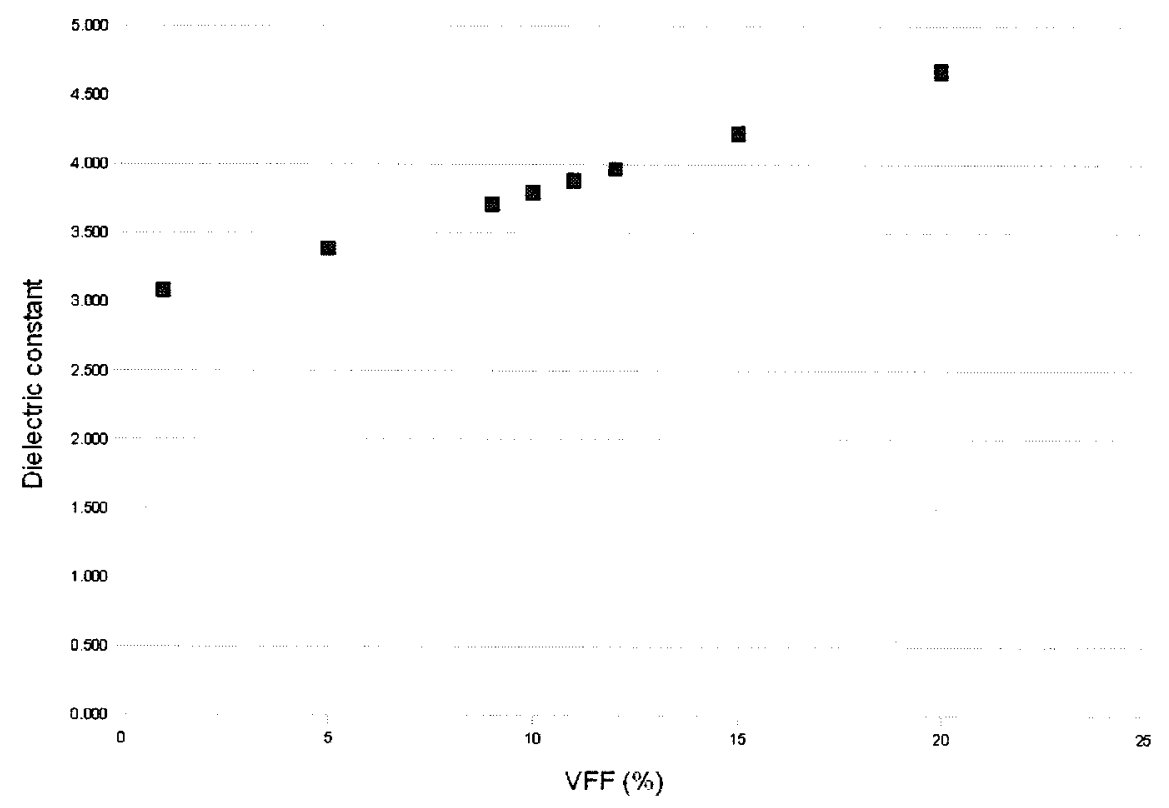

Figure 3.15: Dependence of $\epsilon_{\text {eff }}^{\prime}$ on volume filler fraction (filler particles have a conductivity of $10 \mathrm{~S} / \mathrm{m}$ ) 


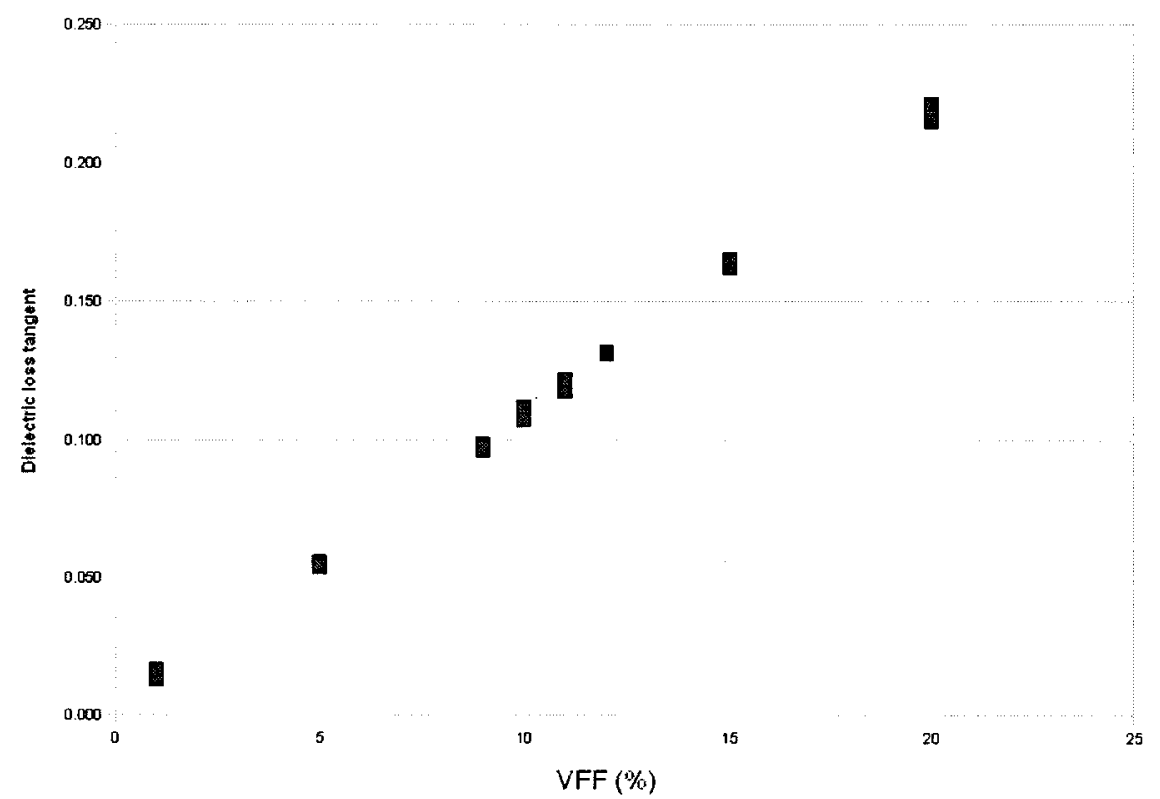

Figure 3.16: Dependence of dielectric loss tangent on volume filler fraction (filler particles have a conductivity of $10 \mathrm{~S} / \mathrm{m}$ )

The simulations using filler particles with a conductivity of $1000 \mathrm{~S} / \mathrm{m}$ gives a percolation threshold around $10-15 \%$ (identified by point at which effective permittivity changes rapidly). Figure 2.10 gives a percolation threshold of around $12 \%$ for particles that have a conductivity of $10^{7} \mathrm{~S} / \mathrm{m}$, a $200 \mathrm{~nm}$ thick shell with a conductivity of $1000 \mathrm{~S} / \mathrm{m}$, and a diameter of $1 \mu \mathrm{m}$. The similarity between the two results demonstrates that the simulation is most likely giving the appropriate results with high volume filler fractions. The results from Figures 3.15 and 3.16 show that large changes in $\epsilon_{\text {eff }}^{\prime}$ and dielectric loss tangent do not occur at the percolation threshold if the filler particles have a low conductivity. It is interesting to note that there is less variation between simulations when the conductivity of the filler particles is lower. 


\subsection{Conclusions}

Numerical electromagnetic solvers are appropriate for predicting the properties of composite materials. The results predicted by both Bruggeman's and percolation theory matched relatively closely with the results obtained via simulation. This means that numerical electromagnetic solvers are a useful tool for designing heterogeneous materials consisting of an insulating matrix with conductive fillers. Furthermore, numerical electromagnetic solvers should allow for more complex material to be modeled than is possible using analytical routes. 


\section{Chapter 4}

\section{Microwave Material Characterization}

\section{Theory}

\subsection{Introduction}

Microwave material characterization is a well established field. There is a significant amount of literature about different techniques for measuring the microwave properties of material. One excellent book on material characterization is Microwave Electronics: Measurement and Materials Characterization []. There have been numerous review papers published discussing specific techniques and their advantages and disadvantages $[1$,$] . Microwave material characterization techniques can be$ broken into two categories, resonant and non-resonant techniques.

Resonant techniques generally measure a material's electrical properties by measuring the resonant frequency and unloaded quality factor of a resonant structure with and without the material under test. Then the material properties can be extracted from the shift in resonance frequency and the change in quality factor. This method yields the most accurate results, but material properties can usually only be measured at a single frequency point. 
Non-resonant methods generally determine material properties via reflectiontransmission measurements. Non-resonant methods are less sensitive than resonant methods, but relatively broadband measurements of material properties can be made.

The first topic to be discussed is the free space transmission-reflection material characterization method. Next, the theory behind resonant perturbation measurement techniques is discussed. Perturbation theory is followed by a brief discussion on dielectric resonators. Next, split post dielectric resonators and single post dielectric resonators are discussed. Lastly, the method to extract the unloaded quality factor of a device using a vector network analyzer is presented.

\subsection{Free Space Transmission-Reflection}

Free space measurement techniques have several advantages [ ]. Some measurement apparatus generate higher-order modes at the air-dielectric interface when measuring composite materials with inhomogeneities, and this can cause errors in the measurements. However, the free space transmission-reflection technique does not suffer from this problem. Another advantage is that free space measurements are contactless. This allows for nondestructive measurements and removes many possible sources of error. Measuring materials that require extreme conditions (eg. superconductors, plasma and liquid metals.) becomes much easier since the measurement apparatus does not need to have contact with the material. Materials do not need to be machined to fit the test apparatus which eliminates some sources of error.

The setup for measuring material properties using a free space transmissionreflection setup is shown in Figure 4.1. This measurement setup assumes that the sample is infinite in size laterally and has a linearly polarized plane wave normally incident. The parameters used in in order to determine the permittivity $\left(\epsilon_{r}\right)$ and 
permeability $\left(\mu_{r}\right)$ of the sample are as follows:

$S_{11}$ is the input reflection coefficient

$S_{21}$ is the forward transmission coefficient

$\gamma$ is the propagation constant of the wave

$\lambda$ is the wavelength of the wave

$\Gamma$ is the reflection coefficient at the air-sample interface

$T$ is the transmission coefficient

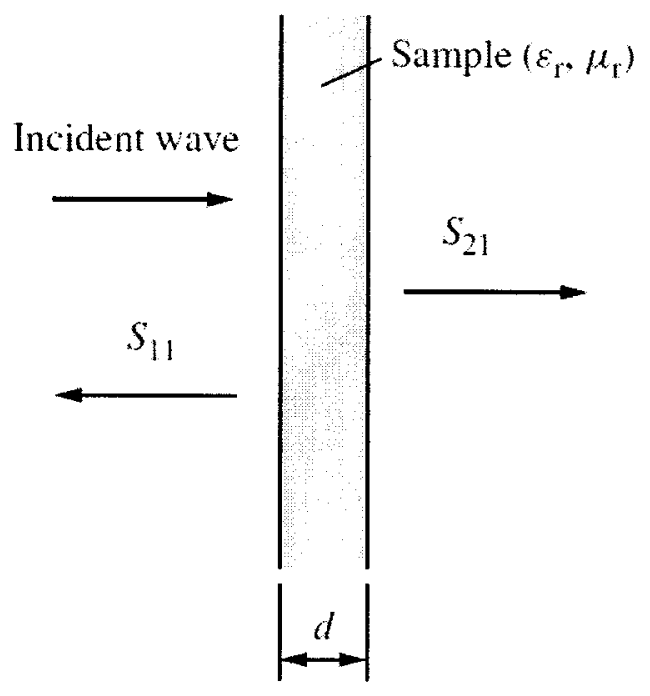

Figure 4.1: Diagram of planar sample [ ]

$$
\gamma_{0}=\frac{j 2 \pi}{\lambda_{0}}
$$

The following equations can be used to extract the material properties of the sample $[\cdots]$

$$
\begin{aligned}
& K=\frac{S_{11}^{2}-S_{21}^{2}+1}{2 S_{11}} \\
& \Gamma=K \pm \sqrt{K^{2}-1}
\end{aligned}
$$




$$
\begin{gathered}
T=\frac{S_{11}+S_{21}-\Gamma}{1-\left(S_{11}+S_{21}\right) \Gamma} \\
\gamma=\frac{\ln (1 / T)}{d}
\end{gathered}
$$

where $d$ is the thickness of the planar sample

$$
\begin{aligned}
& \epsilon_{r}=\frac{\gamma}{\gamma_{0}}\left(\frac{1-\Gamma}{1+\Gamma}\right) \\
& \mu_{r}=\frac{\gamma}{\gamma_{0}}\left(\frac{1+\Gamma}{1-\Gamma}\right)
\end{aligned}
$$

\subsection{Resonant Perturbation Theory}

A resonant perturbation method determines material properties by measuring the change in resonant frequency and change in quality factor when the material under test is added to the resonant cavity. Cavity perturbation methods have a high level of accuracy and sensitivity which makes them perfect for measuring thin film samples. There are three types of cavity perturbations: cavity-shape, wall-impedance, and material perturbation. The type that is discussed here is material perturbation.

Small-object perturbation is a method for material characterization whereby a small part of the original material gets replaced by a new material with unknown electrical properties as shown in Figure 4.2. The full derivation of the equations from Maxwell's equations is unnecessary here and if the reader would like to go through the full derivation it can be found in Microwave Electronics: Measurement and Materials Characterization [ ]. 

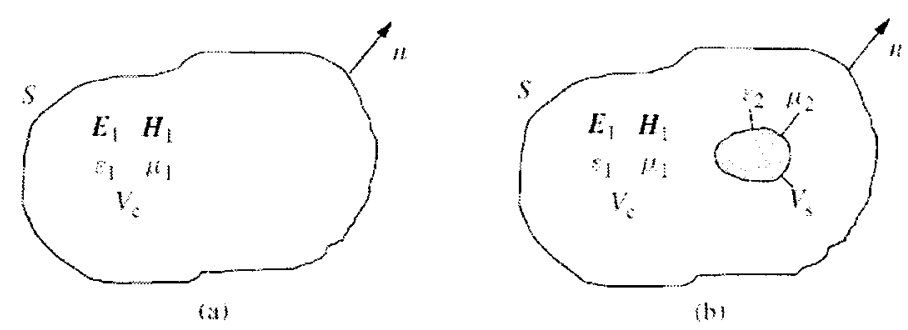

Figure 4.2: Small-object material perturbation. (a) Original material in cavity and (b) Perturbed cavity [ ]

Permittivity measurements are of concern here, so the equations for determining complex permittivity will be discussed and the equations for determining complex permeability will be ignored. Consider a resonant cavity construct with perfectly conducting walls that enclose a surface $\mathrm{S}$, and has a volume V. There are four assumptions made when Equation (4.8) was derived. First, the material originally in the cavity is lossless. Second, the sample is homogeneous and occupies an extremely small volume of the cavity. Third, the distribution of the electromagnetic field outside the sample does not change. Fourth, the material inside the cavity is free space. The cavity perturbation equation is:

$$
\frac{\omega_{2}-\omega_{1}}{\omega_{1}}=-\left(\frac{\epsilon_{r}-1}{2}\right) \frac{\iiint_{V_{S}} \vec{E}_{1} \cdot \vec{E}_{2} d V}{\iiint_{V_{C}}\left|\vec{E}_{1}\right|^{2} d V}
$$

where,

$\omega_{1}$ is the angular resonant frequency of the empty cavity

$\omega_{2}$ is the angular resonant frequency of the cavity with the material under test

$\epsilon_{r}$ is the permittivity of the material under test

$V_{C}$ is the volume of the cavity

$V_{S}$ is the volume of the sample

$\vec{E}_{1}$ is the electric field in the cavity 
$\vec{E}_{2}$ is the electric field in the material under test

The resonant frequency of a resonant cavity is given by a complex angular frequency as shown in Equation (4.9). The quality factor of the resonator is given by Equation (4.10).

$$
\begin{gathered}
\omega=\omega_{r}+j \omega_{i} \\
Q=\frac{\omega_{r}}{2 \omega_{i}}
\end{gathered}
$$

If one assumes that $\omega_{r 1} \approx \omega_{r 2}, \omega_{i} \ll \omega_{r}$, and $Q_{1} \gg 1$ then:

$$
\begin{gathered}
\frac{\omega_{2}-\omega_{1}}{\omega_{1}}=\frac{\left(\omega_{r 2}-\omega_{r 1}\right)+j\left(\omega_{i 2}-\omega_{i 1}\right)}{\omega_{r 1}\left(1+j \frac{\omega_{i 1}}{\omega_{r 1}}\right)} \\
\frac{\omega_{2}-\omega_{1}}{\omega_{1}} \approx\left[\left(\frac{f_{2}-f_{1}}{f_{1}}\right)+j\left(\frac{1}{2 Q_{2}}-\frac{1}{2 Q_{1}}\right)\right]\left(1-j \frac{1}{2 Q_{1}}\right)
\end{gathered}
$$

where,

$Q_{1}$ is the unloaded quality factor of the empty cavity

$Q_{2}$ is the unloaded quality factor of the cavity with the sample

$$
\frac{\omega_{2}-\omega_{1}}{\omega_{1}} \approx\left(\frac{f_{2}-f_{1}}{f_{1}}\right)+j\left(\frac{1}{2 Q_{2}}-\frac{1}{2 Q_{1}}\right)
$$

Combining Equations (4.8) and (4.13) gives:

$$
\left(\frac{f_{2}-f_{1}}{f_{1}}\right)+j\left(\frac{1}{2 Q_{2}}-\frac{1}{2 Q_{1}}\right)=-\left(\frac{\epsilon_{r}-1}{2}\right) \frac{\iiint_{V_{S}} \vec{E}_{1} \cdot \vec{E}_{2} d V}{\iiint_{V_{C}}\left|\vec{E}_{1}\right|^{2} d V}
$$


Equation 4.14 can be rewritten as Equations (4.15), (4.16), and (4.17).

$$
\begin{gathered}
2\left(\frac{f_{1}-f_{2}}{f_{1}}\right)=\left(\epsilon_{r}^{\prime}-1\right) C \\
\frac{1}{Q_{2}}-\frac{1}{Q_{1}}=\epsilon_{r}^{\prime \prime} C \\
C=\frac{\iiint_{V_{S}} \vec{E}_{1} \cdot \vec{E}_{2} d V}{\iiint_{V_{C}}\left|\vec{E}_{1}\right|^{2} d V}
\end{gathered}
$$

\subsection{Dielectric Resonators}

Since the discovery of materials with relatively high permittivity, temperature stability and low dielectric loss tangent, dielectric resonators have become commonly used in all sorts of microwave circuits. The type of dielectric resonator of importance here is the cylindrical disk dielectric resonator. The first resonant mode of a cylindrical dielectric resonator is the $T E_{01 \delta}$ mode and can be seen in Figure 4.3.

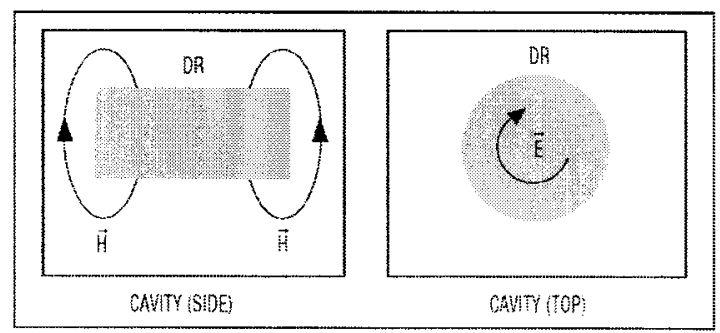

Figure 4.3: $T E_{01 \delta}$ mode of a dielectric puck resonator [ ]

There are a few important parameters when dealing with dielectric resonators. The dielectric constant and the quality factor of the dielectric resonator are the two obvious parameters. The quality factor is simply $1 / \tan \delta$, where $\tan \delta$ is the dielectric loss tangent of the ceramic used to make the dielectric resonator. The temperature 
coefficient of a dielectric resonator is a measure of the shift in resonant frequency with temperature. This includes the temperature coefficient of the dielectric and the thermal expansion of the dielectric [ ]. The $T E_{01 \delta}$ resonant frequency of an isolated cylindrical dielectric resonator is approximated by the following equation [ ]:

$$
f_{\circ}(G H z) \approx \frac{8.553}{\sqrt{\epsilon_{r}}\left(\frac{\pi}{4} D_{r}^{2} L_{r}\right)^{1 / 3}}
$$

where:

$D_{r}$ is the resonator diameter in inches

$L_{r}$ is the resonator length in inches

$\epsilon_{r}$ is the resonator dielectric constant

The above equation will only give an approximate value of the resonant frequency. In order to obtain more accurate results a numerical electromagnetic solver needs to be utilized. The effects of metallic and dielectric materials in close proximity are shown in Figure 4.4. This can be used to tune the dielectric resonator up to $\pm 20 \%$, however it is suggested to not tune the resonant frequency more than $\pm 5 \%$ so as to not degrade the unloaded Q and temperature coefficient [ ]. 


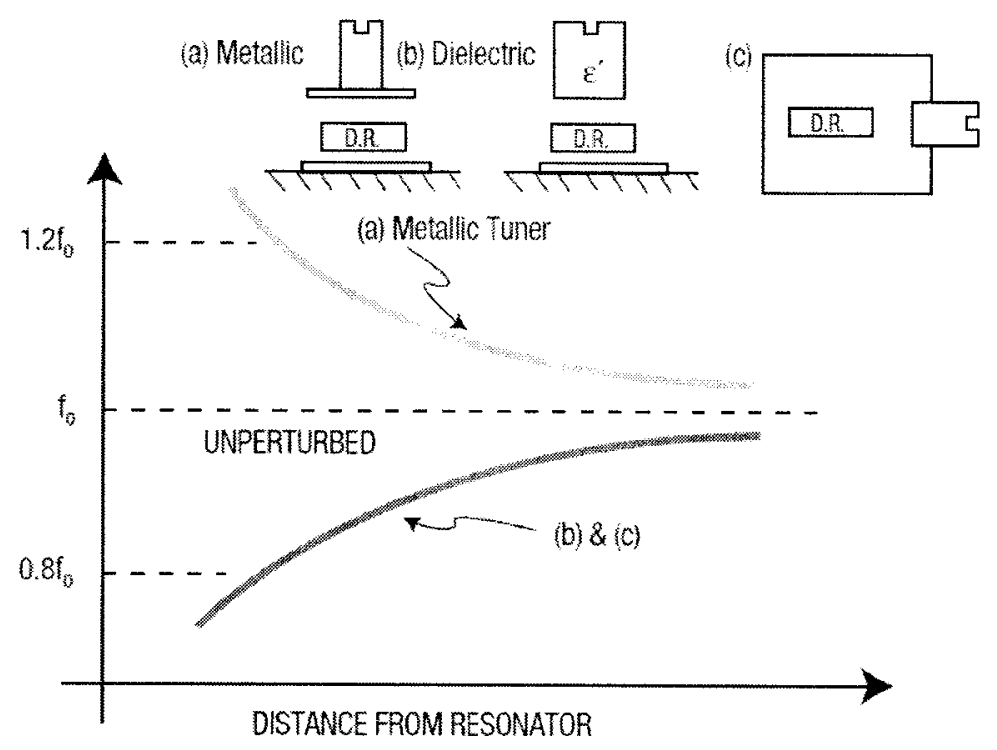

Figure 4.4: Effects of metallic wall and dielectric material on resonant frequency [ ]

The last issue that needs to be discussed is coupling to the dielectric resonator. A few coupling methods are shown in Figure 4.5. Figure 4.5(a) shows magnetic coupling via a conductive wire loop. Figure 4.5(b) shows magnetic coupling to a microstrip line. Figure 4.5 (c) shows magnetic coupling to a straight section of wire.

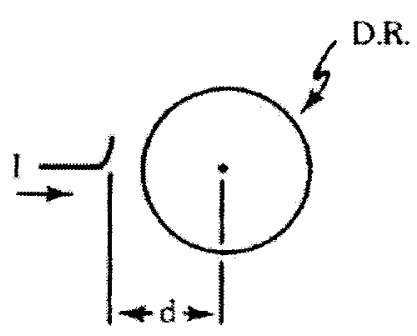

(a)

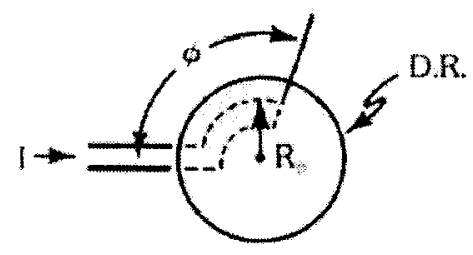

(b)

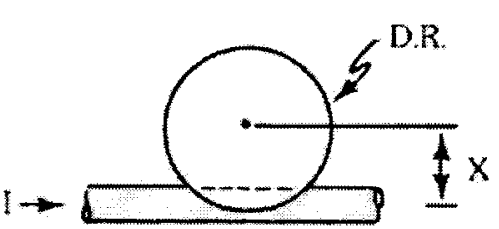

(c)

Figure 4.5: Excitation of dielectric resonators [ ]

The external quality factor is heavily dependent upon how one couples to the dielectric resonator. The external quality factor of the side coupled resonator shown in Figure 4.5(a) will decrease as $d$ decreases. While the external quality factor of 
the microstrip coupled resonators shown in Figures 4.5(b) and 4.5(c) will decrease as the dielectric resonator approaches the substrate vertically. For the radially coupled resonator the $\mathrm{Q}$ will decrease with increasing angle and will be at a maximum when the length of the line is close to a quarter wavelength in the substrate [ ].

\subsection{Split-Post Dielectric Resonator (SPDR)}

SPDRs (shown in Figure 4.6) have become rather popular recently. This is because advances in numerical electromagnetic simulation software has allowed for more complex structures to be designed and utilized. This means that the structure can now be designed to suit the material needs not the other way around. The SPDR was developed specifically to measure laminar dielectrics and has proven ideal for measuring thin-film materials [1]. Currently the SPDR is considered one of the most accurate techniques for measuring complex permittivity. The uncertainty in measurement of the real part of the permittivity is approximately $0.3 \%$. Samples with appropriate thickness can have the dielectric loss tangent resolved to approximately $2 \times 10^{-5}$. The Q-factor of the apparatus can be measured with an accuracy of $1 \%$ [ ]. The biggest contributing factor to uncertainty using this technique comes from uncertainty in the thickness of the material under test [ ] 


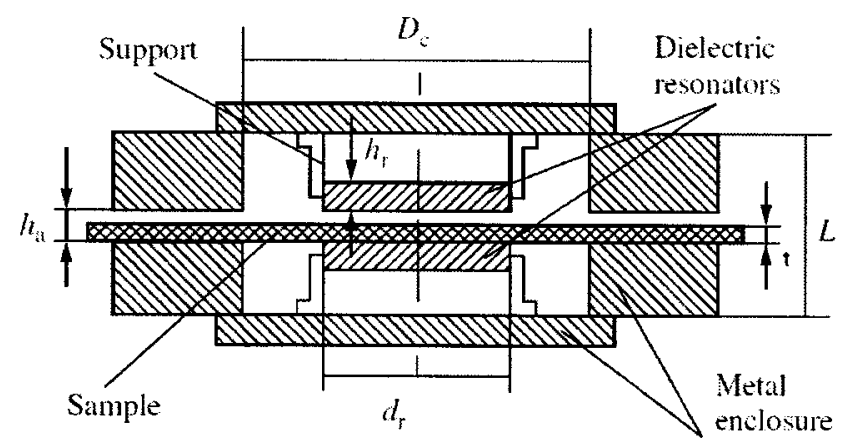

Figure 4.6: Cross section of an SPDR [ ]

The permittivity of the material under test is given by:

$$
\epsilon_{r}^{\prime}=1+\frac{f_{0}-f_{1}}{t f_{0} K_{\epsilon}\left(\epsilon_{r}^{\prime}, t\right)}
$$

where

$t$ is the thickness of the sample

$f_{0}$ is the resonant frequency of the empty fixture

$f_{1}$ is the resonant frequency of the fixture with the material

$K_{\epsilon}$ is a fitting constant with the value obtained via simulation

The dielectric loss tangent of the material is determined from the following equation:

$$
\tan \delta=\left(Q^{-1}-Q_{\text {apparatus }}^{-1}\right) / p_{e s}
$$

where, $Q_{\text {apparatus }}$ is the unloaded quality factor of the apparatus without the material under test, $Q$ is the unloaded quality factor of the apparatus with the material under test, and $p_{e s}$ is: 


$$
p_{e s}=t \epsilon_{r}^{\prime} K_{1}\left(\epsilon_{r}^{\prime}, t\right)
$$

where, $K_{1}$ is determined using an electromagnetic simulator.

\subsection{Single-Post Dielectric Resonator(SiPDR)}

The single post dielectric resonator (shown in Figure 4.7) is almost the same structure as the SPDR, but was developed to characterize very lossy materials. This technique is intended to measure the conductivity or dielectric loss tangent of a material and cannot measure the real part of the permittivity. This technique follows the same perturbation theory as the SPDR, however the sample under test is placed in an area with a relatively small electric flux density. By placing the sample in this area the resonant frequency and quality factor will be influenced much less by the sample. The desired placement of the sample holder is in an area of low enough electric flux density that the dielectric constant of the material will have negligible effect on the resonant frequency and quality factor of the cavity. In this placement the quality factor will only be affected by the dielectric loss tangent of the sample, and the resonant frequency will only shift if the sample has a high enough conductivity (or an extremely high dielectric loss tangent).

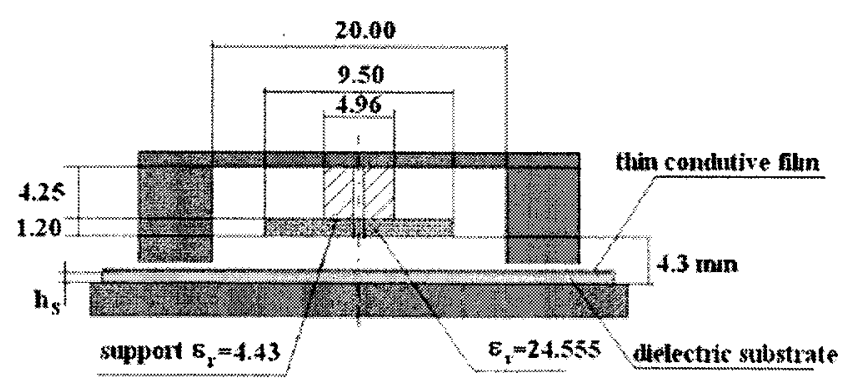

Figure 4.7: Cross section of SiPDR [ ] 


\subsection{Unloaded Q Measurements Using a Vector Network Analyzer}

There are times when one requires the unloaded quality factor of a resonant circuit. Unfortunately such a parameter cannot be measured directly using a vector network analyzer. Fortunately, a paper entitled Q-Factor Measurement with Network Analyzer [ ] was published. An example of a measurement made using a VNA is shown in Figure 4.8 with all the variables used in the following equations labeled. The circle seen on the Smith chart is what the frequency response of a resonant circuit looks like.

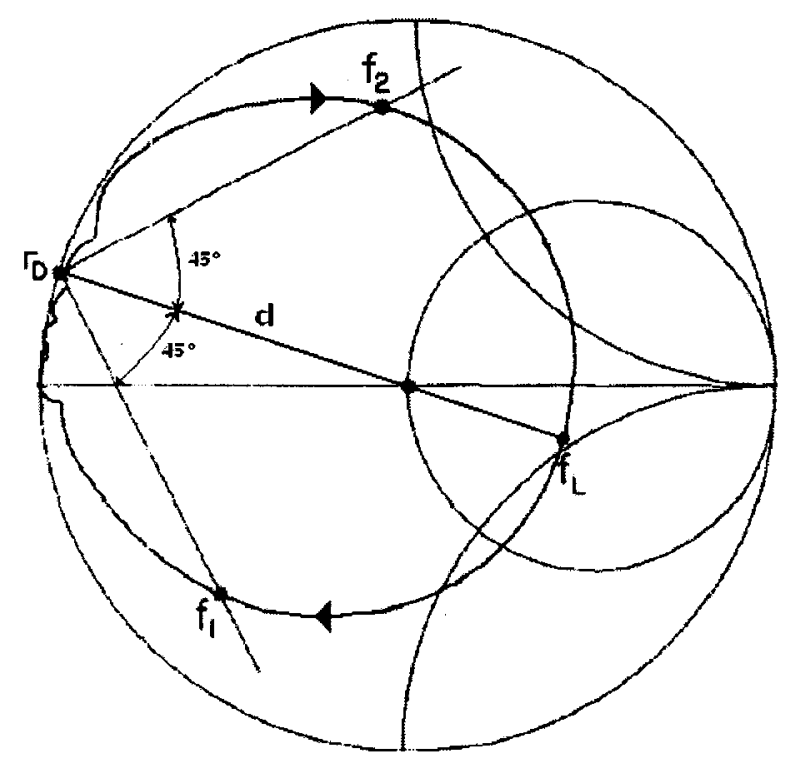

Figure 4.8: Input reflection coefficient of a resonant circuit with swept frequency [ ] 
The coupling coefficient is given by the following equation:

$$
\kappa=\frac{d}{2-d}
$$

where, $d$ is the diameter of the circle shown in Figure 4.8. The loaded quality factor of the circuit is given by Equation 4.23

$$
Q_{L} \approx \frac{f_{L}}{f_{1}-f_{2}}
$$

where,

$f_{L}$ is the loaded resonant frequency of the circuit

$f_{1}$ and $f_{2}$ are the upper and lower $3 \mathrm{~dB}$ frequencies from resonance, respectively The unloaded quality factor is then given by:

$$
Q_{0}=Q_{L}(1+\kappa)
$$

\subsection{Summary}

This chapter presented the theory behind the material characterization techniques used in this work. The chapter began by presenting the theory used to extract material properties for a free-space transmission-reflection techniques. Next, the theory known as perturbation was discussed. This theory is the basis for the technique used in this work to measure the microwave properties of materials. Next, an overview of dielectric resonators was given. Following the discussion on dielectric resonators, two material characterization apparatus that use dielectric resonators were discussed. These two 
measurement apparatus are the basis for the microwave material characterization apparatus developed for this work. Finally, the chapter concluded with a discussion on how to extract the unloaded quality factor of a circuit using a vector network analyzer. 


\section{Chapter 5}

\section{Novel Open Single Post Dielectric Resonator (OSiPDR)}

\subsection{Introduction}

There are a vast number of characterization techniques that have been developed over the years. Each characterization technique has certain strengths and weaknesses. In order to choose an appropriate characterization method it is important to know exactly what type of material needs to be characterized. The characterization apparatus for this work must meet these requirements:

- be able to characterize a film deposited on a substrate with a thickness between $1-100 \mu m$

- does not need to be able to characterize magnetic materials;

- must be able to characterize very lossy materials;

- must be able to apply light to samples;

- measurements must be easily repeatable; 
- preferably a contactless and non-destructive method;

- measure materials with an isotropic dielectric constant;

- measure materials with a dielectric constant between 2 and 100 .

Resonant characterization techniques are the most sensitive techniques and can measure thin-film materials with relative ease and accuracy. Light can be applied to the sample by using a technique that does not require the resonant cavity to be fully enclosed. Dielectric resonators (DR) contain the majority of the electric field inside the DR, which means that an apparatus that utilizes a DR would be appropriate and should not radiate much, if any, of the energy. Recently, there has been interest in the SPDR because it is the most accurate apparatus to characterize the microwave properties of thin film materials. The SPDR uses DRs above and below the sample which is not useful for this work. If the top DR is removed and the metal cavity is extended in height, the resonant cavity should function very similar to the SPDR. However, there will be reduced sensitivity because more of the electric field will be contained within the single DR. This structure has the advantage that a second sample holder can be added further above the dielectric resonator. This second position has significantly less electric flux density which means that the resonant frequency $\left(f_{\circ}\right)$ and unloaded quality factor $\left(Q_{\circ}\right)$ are less sensitive to the properties of the sample. The real part of the permittivity cannot be measured when the sample is in the top position, however the loss tangent of lossy materials can be easily measured. A schematic representation of the proposed measurement apparatus is shown in Figure 5.1. 


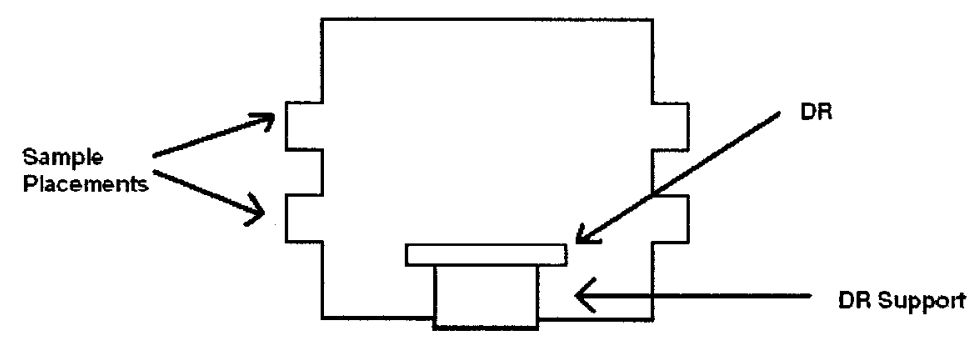

Figure 5.1: Cross section of OSiPDR (All components are cylindrical).

The first issue discussed is the design of the cavity. This section discusses the determination of initial parameters, the simplification of the simulation setup, and the optimization of the cavity. The next section discusses how one extracts material properties using the OSiPDR. Next, issues that may affect the accuracy of measurements are discussed. After discussing accuracy, the tuning and verification of the fabricated cavity is presented.

\subsection{Design of Cavity}

\subsubsection{Determination of Cavity Specifications}

Resonant structures can only measure material properties at a single frequency point, which means that point must be chosen with care. There are many factors that must be accounted for when choosing a frequency point.

As measurement frequency decreases the necessary size of the resonant cavity increases which means the sample size increases. A larger sample size requires a larger substrate to put the sample on. The larger the substrate has to be laterally, the thicker the substrate must be so as not be too fragile. A thicker substrate contributes more to losses in the resonant cavity, which decreases the sensitivity of the cavity for measuring the dielectric loss of a material. It is possible to purchase substrates that 
have excellent dielectric properties. Unfortunately, these tend to be more expensive making it impractical if a large number of samples need to be characterized. There is one cheap alternative that should work well. Glass cover slips that are used for microscopy are a good option. They are relatively thin and cheap. The largest size that can be found are $25 \mathrm{~mm}$ in diameter. Some of the material can be costly, so it is ideal to use a higher frequency for measurements which would require less material. However, once sizes get too small the precision required to fabricate the measurement apparatus becomes prohibitive. Also, the higher in frequency one goes the more difficult it is to find a dielectric resonator with a reasonable quality factor. All these reasons leaves a frequency around $8 \mathrm{GHz}$ as a good choice.

The materials obtained for the design of the OSiPDR are as follows:

- D8371-0265-119 DR from Trans Tech

- D4-0236-092 DR Support from Trans Tech

- RG405 conformable coax cable

- Loctite 409 adhesive

- 25mm Bellco Brand round cover slips (Corning 0211 alkali zinc borosilicate glass)

The electrical properties of the DR, DR support, adhesive, and cover slips are given in Table 5.1. 
Table 5.1: Properties of dielectric resonator and support

\begin{tabular}{|c|c|c|c|}
\hline Component & Tan $\delta$ & $\epsilon^{\prime}$ & Frequency (GHz) \\
\hline Dielectric Resonator [ ] & 0.000167 & $35.5 \pm 1$ & 8 \\
\hline DR Support [ ] & 0.0002 & 4.5 & - \\
\hline Loctite 409 [ ] & 0.02 & 2.3 & 0.00001 \\
\hline Corning 0211 Glass [ ] & 0.0046 & 6.7 & 0.001 \\
\hline
\end{tabular}

Since the microwave properties of the Corning 0211 glass cannot be found, the low frequency properties are used. The dielectric constant and loss tangent of the cover slips can be measured later.

\subsubsection{Simulation Setup and Simplification}

The simulations in this work were performed using Ansoft High Frequency Structure Simulator (HFSS). The simulator can perform three types of simulation, and the two simulation types used in this work were: driven modal, and eigenmode. The driven modal simulation calculates the s-parameters based upon each mode of excitation, while the eigenmode simulation determines the resonant frequencies of a structure.

The cavity was designed to resonate in the $T E_{01 \delta}$ mode, and was optimized for the $f_{\mathrm{o}}$ and $Q_{\mathrm{o}}$. The frequency in which the resonant cavity resonates at was of little importance during design as long as it was close to $8 \mathrm{GHz}$. If the $Q_{0}$ of the resonant cavity was too low it would have been more difficult to measure the loss of low-loss materials. Therefore, the $Q_{0}$ of the cavity was optimized during design. 
Before simulation could be done a few initial parameters of the resonant cavity were determined. A basic rule of thumb is that the $Q_{\circ}$ of the resonant cavity will be lower the closer the metal walls are to the DR. Since the cover slips were $25 \mathrm{~mm}$ in diameter, the diameter of the cavity must be smaller than $25 \mathrm{~mm}$, this made $20 \mathrm{~mm}$ for the diameter as a good starting point. The height of the metal cavity was chosen to be $15 \mathrm{~mm}$, which provided enough space for at least one DR support, one DR, and both sample holders. An indent $0.2 \mathrm{~mm}$ deep was placed in the center of the resonant cavity because it made it easier to place the DR support in the right position. The diameter and height of the DR was $6.73 \mathrm{~mm}$ and $3.02 \mathrm{~mm}$ respectively. The diameter and height of the DR support was $5.99 \mathrm{~mm}$ and $2.34 \mathrm{~mm}$ respectively.

The majority of the simulations performed were eigenmode simulations because the $f_{\circ}$ and $Q_{\circ}$ of the cavity were readily available. Also, the eigenmode simulation allowed for the complexity of the simulated structure to be simplified. The first issue to be addressed was speeding up simulation time. This was done by using a few key properties of the structure and the resonant mode of interest. The first simulations done were for a comparison between two different structures. The first structure consisted of the full resonant structure as shown in Figure 5.2(a). The second structure used perfect electric conductors (PEC) on the boundary where it was 'sliced' from the structure, and is shown Figure 5.2(b). The PEC boundary effectively mirrored the rest of the structure for modes in which the electric field was normal to the PEC. Since the $T E_{01 \delta}$ mode was the only mode of interest this was appropriate. The PEC boundary allowed modes to exist that were not real and it prevented other modes from appearing in simulation. However, these erroneous modes and the missing modes did not have a significant effect on the accuracy of the $f_{\circ}$ and $Q_{\circ}$ of the first mode. The $f_{\circ}$ and $Q_{\circ}$ for the full cavity were $7.97577 \mathrm{GHz}$ and 5977.8 respectively. The $f_{\circ}$ and $Q_{\circ}$ of the slice of the cavity were $7.90076 \mathrm{GHz}$ and 
5811.96 respectively. The variation in $f_{0}$ was $0.94 \%$, and the variation in the quality factor was $2.77 \%$.

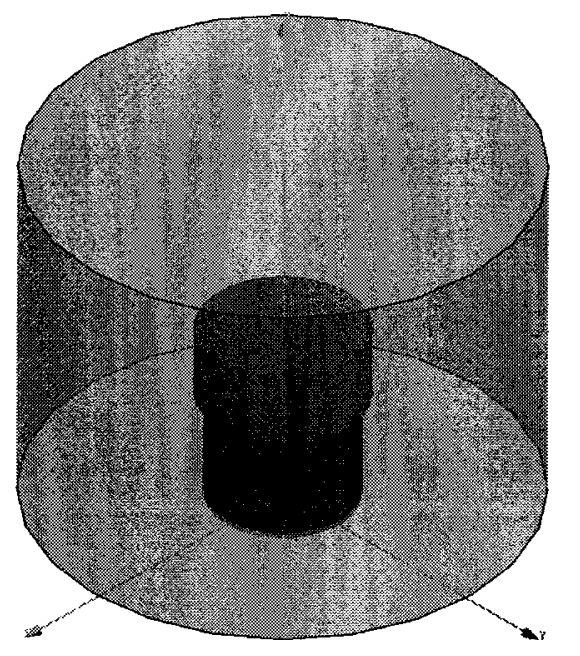

(a) Full cavity

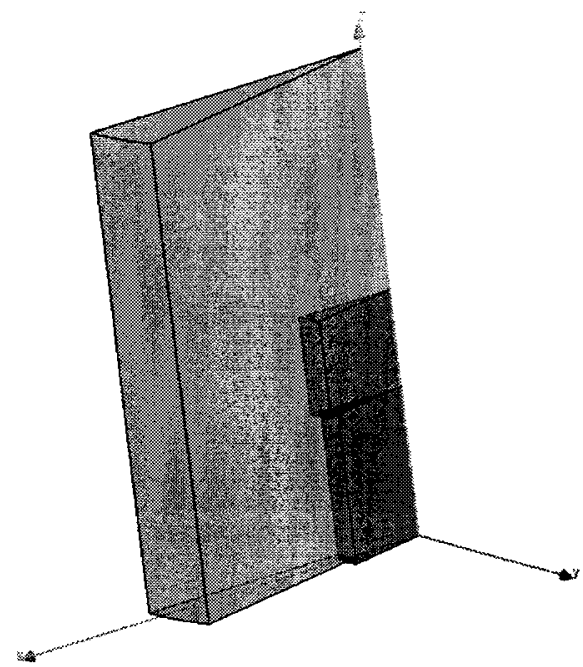

(b) Slice of cavity

Figure 5.2: Models of resonant cavity

The electric field distribution in both simulations was examined to further confirm that the two simulations gave relatively similar results. The electric field distributions are shown in Figures 5.3 and 5.4, and it can be seen that the excited mode was the $T E_{01 \delta}$ mode. 

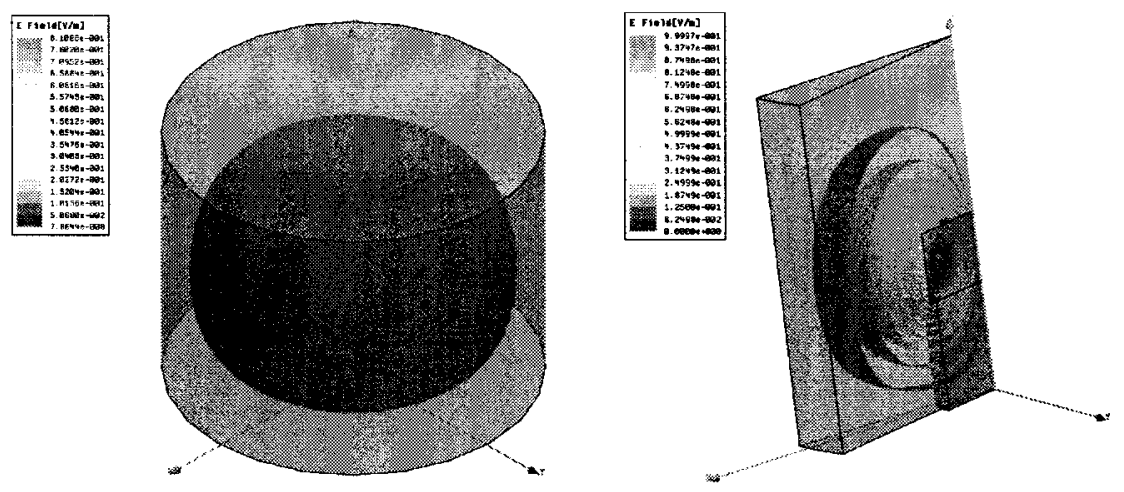

(a) Magnitude representation of electric (b) Magnitude representation of fields in full cavity electric fields in slice of cavity

Figure 5.3: Magnitude of electric field in full cavity
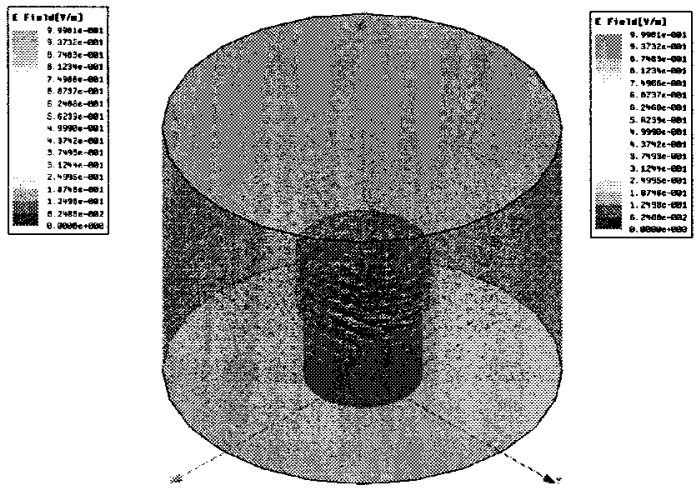

Figure 5.4: Vector representation of electric fields in full cavity

\subsubsection{Sizing of Cavity}

From this point in the design, the $Q_{0}$ of the cavity was optimized. This was because the $f_{0}$ could not have much of an effect on the accuracy of the measurements, while a small $Q_{\circ}$ would have decreased the accuracy of the loss measurements. The first issue to be addressed was how many dielectric supports should be used. The $Q_{0}$ of the cavity with one, two, and three supports is shown in Figure 5.5. From the following 
figure one can see that using more than two supports would have had a minimal effect on increasing the $Q_{\mathrm{o}}$ of the cavity.

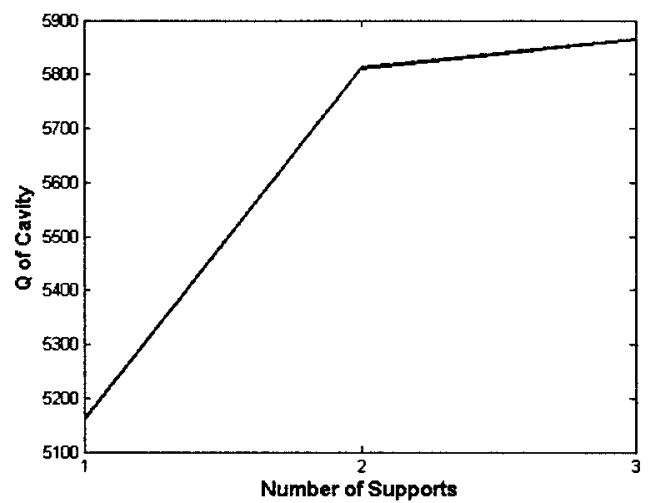

Figure 5.5: Dependence of the $\mathrm{Q}$ of resonator on distance DR is from base of cavity

Next, the resonant cavity was simulated with different diameters and the results are shown in Figure 5.6. One can see that choosing $10 \mathrm{~mm}$ for the radius of the cavity is appropriate and increasing the radius further would have had a minimal effect on the $Q_{\mathrm{o}}$ of the cavity.

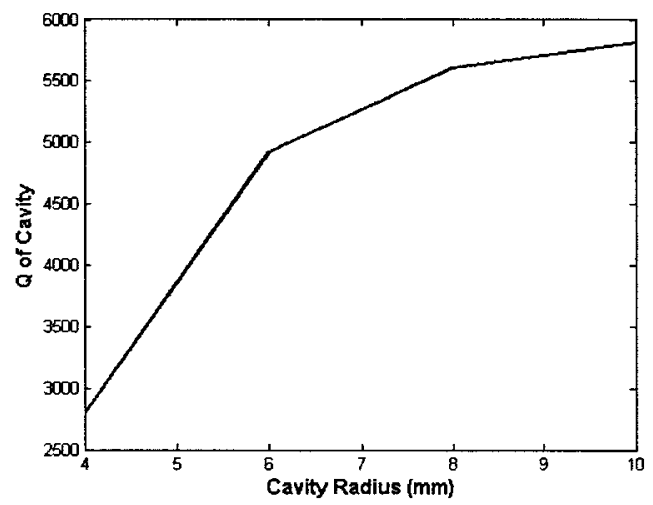

Figure 5.6: Dependence of $Q$ of cavity on diameter of cavity

The height of the cavity was simulated next, and the $Q_{\circ}$ of the cavity versus height is shows in Figure 5.7. It can be seen that increasing the height of the cavity above 
$14 \mathrm{~mm}$ or $15 \mathrm{~mm}$ would have negligible effect on the $Q_{\circ}$ of the cavity. However, since the top of the cavity was to be removed for all of the measurements it was preferable to choose a cavity height that was in excess of $15 \mathrm{~mm}$. Therefore, a height of 19.8 mm was chosen.

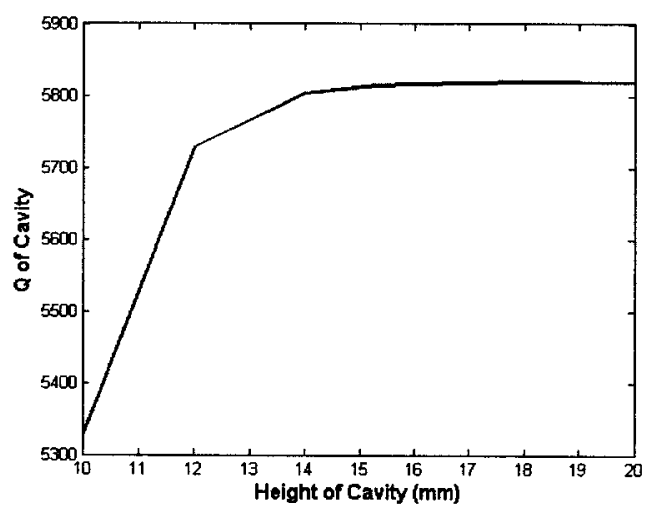

Figure 5.7: Dependence of $\mathrm{Q}$ of cavity on cavity height

With the dimensions of the cavity determined, the next step was to determine the positions of the sample holders. The first sample holder was placed just above the DR because this position had the greatest sensitivity. The height of the sample holder was chosen to be $1.5 \mathrm{~mm}$ because this allowed room for multiple samples to be stacked if needed. The next sample holder was placed in a position that was insensitive to the dielectric constant of the sample. The position was chosen to be where the electric field was significantly decreased, but still strong enough to be sensitive to the loss of the sample. The position of the sample was chosen to be $3 \mathrm{~mm}$ above the top of the first sample holder. Figure 5.8 demonstrates that the permittivity of the sample will have relatively little effect on the properties of cavity. While, Figure 5.9 shows that the conductivity of the sample will affect both the $f_{\mathrm{o}}$ and $Q_{\mathrm{o}}$ of the cavity 


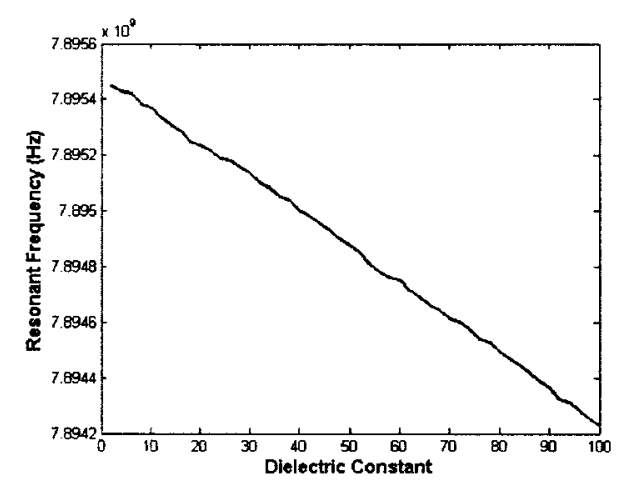

(a) $f_{r}$ of cavity

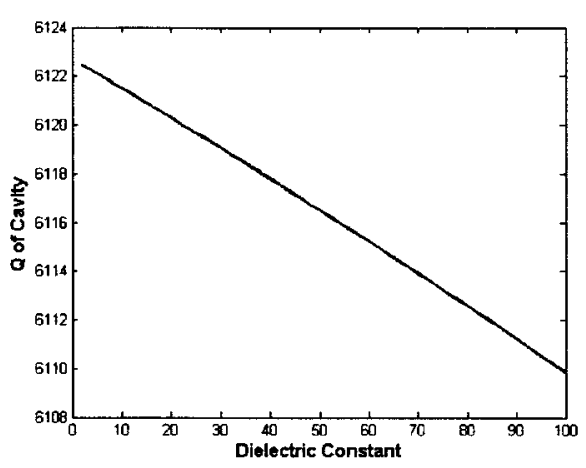

(b) Q of cavity

Figure 5.8: Properties of cavity when a material with a dielectric constant varying from 2 to 100 and a dielectric loss of 0.001 is placed in the top holder

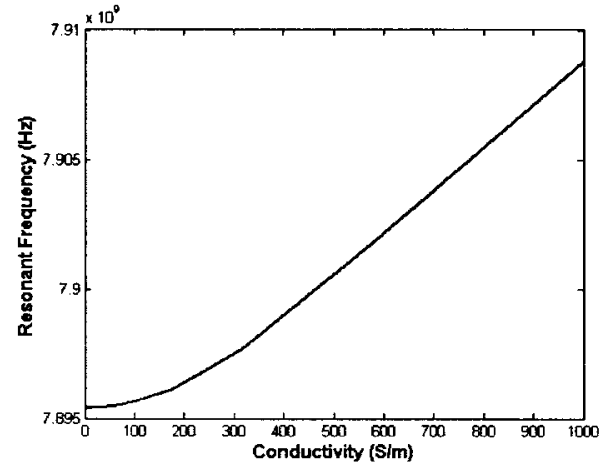

(a) $f_{r}$ of cavity

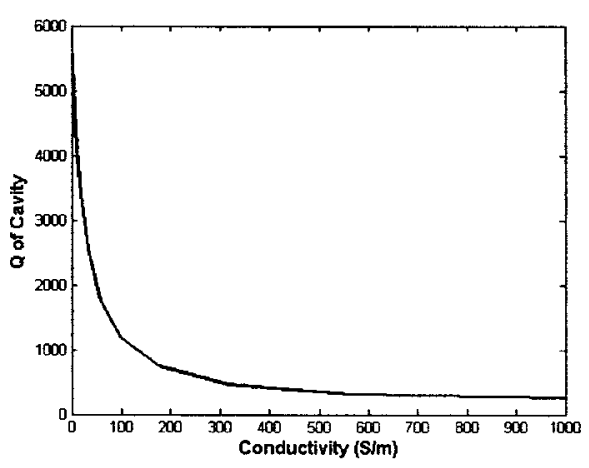

(b) Q of cavity

Figure 5.9: Properties of cavity when a material with conductivity varying from $0.001 \mathrm{~S} / \mathrm{m}$ to $1000 \mathrm{~S} / \mathrm{m}$, dielectric constant of 3 , and dielectric loss tangent of 0.001 is placed in the top holder

The next step in designing the cavity was to design the coupling circuit. A coupling loop was chosen as the best method to apply a microwave signal to the cavity. The loop couples easily to the $T E_{01 \delta}$ mode because the magnetic field of the loop and the $T E_{01 \delta}$ mode overlap. The radius of the loop was tuned in order to obtain critical coupling to the $\mathrm{DR}$, which allowed the loaded $\mathrm{Q}$ to be equal to the $Q_{\mathrm{o}}$. In practice 
this was not be the case and the unloaded Q was extracted in the way described in Section 4.7. The coax cable chosen was a conformable RG405 coax with the following properties: inner conductor diameter of $0.51 \mathrm{~mm}$; the polytetraflouroethylene (PTFE) dielectric diameter was $1.68 \mathrm{~mm}$; and the outer conductor had a diameter of $2.16 \mathrm{~mm}$. The conformable cable was chosen because it was the easiest to work with, and the size of the coax was chosen to be as large as was practical. Next, the full structure was constructed in HFSS and is shown in Figure 5.10. A modal simulation was run on the structure with the radius of the coupling loop varying from $1 \mathrm{~mm}$ to $3 \mathrm{~mm}$. A radius of $1 \mathrm{~mm}$ was determined to give an external $\mathrm{Q}$ close enough to the $Q_{0}$ of the structure simulated previously.

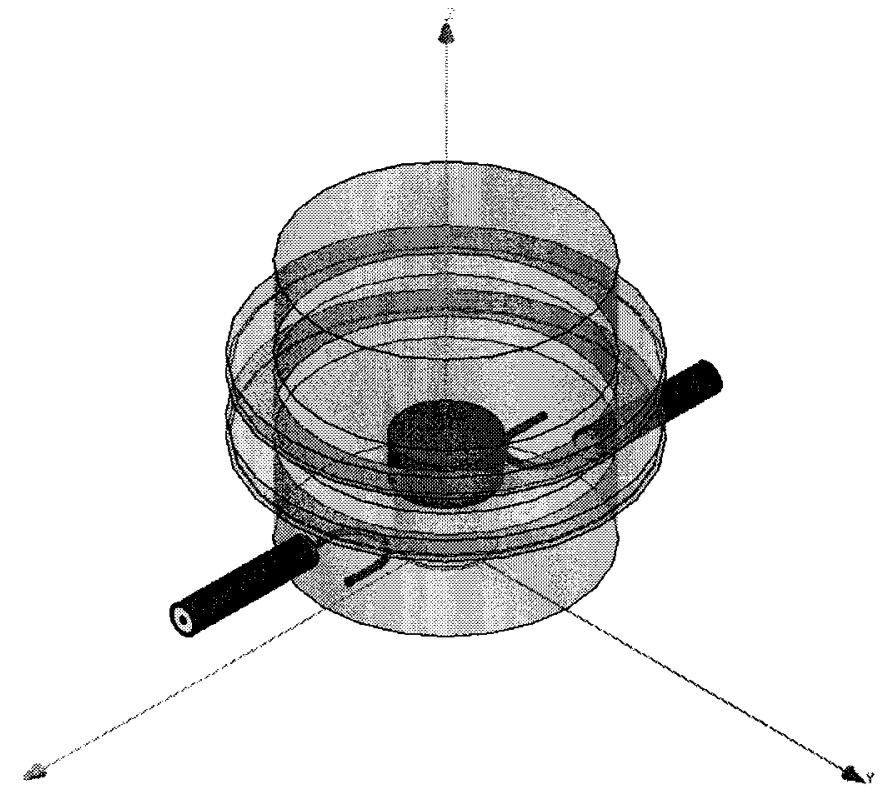

Figure 5.10: Complete resonant cavity simulated in HFSS

To confirm the resonator was able to measure thin samples a few eigenmode simulations were performed with a $10 \mu \mathrm{m}$ thick sample on a glass cover slip (low frequency values were used). The dielectric constant of the sample was swept from 2-100 with a dielectric loss of 0.001 . Next, a simulation of a $10 \mu \mathrm{m}$ thick sample on 
a glass cover slip that had a dielectric loss ranging from 0.001 to 0.1 and a dielectric constant of 3 was simulated. Figures 5.11 and 5.12 shows that the $f_{\circ}$ was only dependent upon the dielectric constant of the sample and that the shift in $f_{0}$ was in the $\mathrm{MHz}$ region which was measurable. The $Q_{\circ}$ of the cavity was dependent upon the dielectric constant and the dielectric loss as expected.

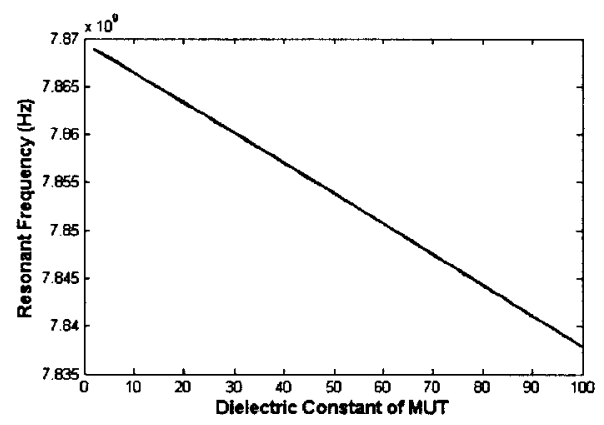

(a) $f_{r}$ of cavity

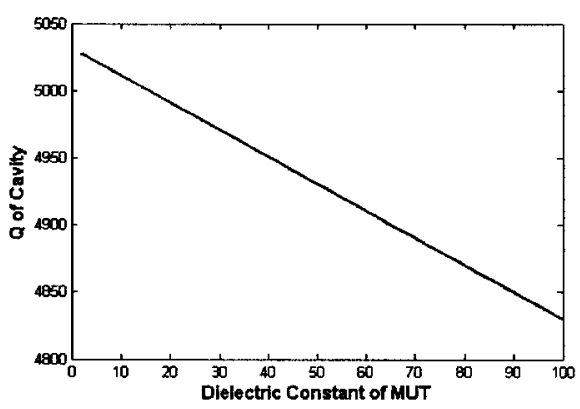

(b) Q of cavity

Figure 5.11: Dependence of cavity properties on dielectric constant of a $10 \mu \mathrm{m}$ thick sample

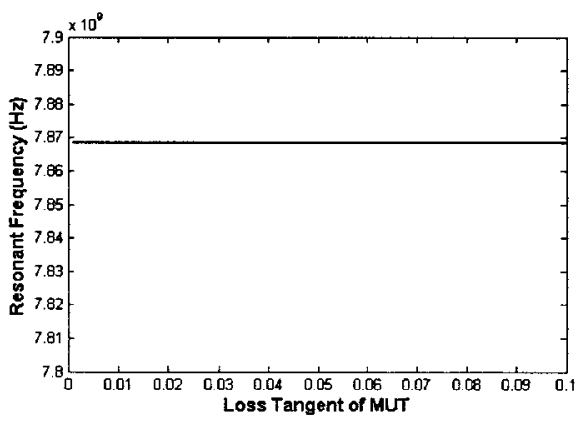

(a) $f_{r}$ of cavity

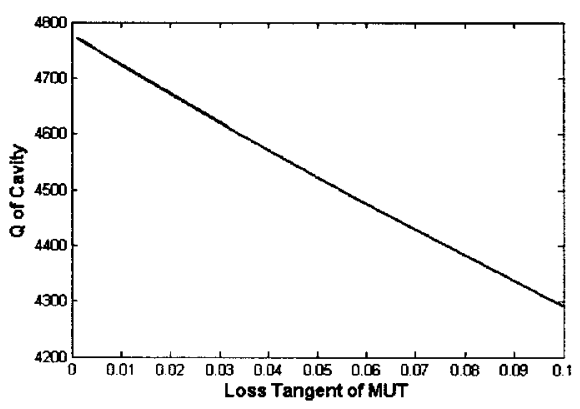

(b) Q of cavity

Figure 5.12: Dependence of cavity properties on dielectric loss of a $10 \mu \mathrm{m}$ thick sample 


\subsection{Material Property Extraction}

This section describes the methodology used to extract the material properties. The same technique can be applied to extract the material properties in either of the sample holder positions. However, the real part of the permittivity cannot be determined when the material is placed in the top holder position. Since there were no medium-high loss samples measured, only the procedure to measure low-medium loss samples is discussed.

The procedure to measure the complex permittivity of a sample is as follows:

1. Measure the properties $\left(f_{\circ}\right.$ and $\left.Q_{\circ}\right)$ of the empty cavity at all the conditions the sample will be measured.

2. Measure the properties of the cavity with the sample at all the desired conditions.

3. Determine the shift in $f_{\mathrm{o}}$ the sample caused.

4. Simulate the empty cavity.

5. Simulate the cavity with a geometrically identical sample as that measured previously. Set the loss of the sample low and simulate the sample with a range of dielectric constants.

6. Using linear interpolation, determine what dielectric constant in simulation gives the same shift in $f_{\mathrm{o}}$ measured.

7. Use Equation (4.15) to determine the filling factor C.

8. If the $Q_{\circ}$ of the cavity is less than 5249 , then remove the loss due to humidity from the measured $Q_{\mathrm{o}}$ value. 
9. Use the $\mathrm{C}$ value calculated, Equation (4.16), the $Q_{\mathrm{o}}$ of the empty cavity, and the $Q_{0}$ of the cavity with the sample to determine the imaginary part of the permittivity.

Measuring the sample on a substrate is almost the same as measuring just the sample. The only difference is that any time that the empty cavity is measured or simulated, the cavity with the substrate is measured or simulated.

\subsection{Accuracy}

There are many factors that can have an effect on the accuracy of the measured material properties. This section will discuss some of the parameters that affect the accuracy of the measurement of $\epsilon_{r}^{\prime}$. The loss of the materials measured in this work is of minimal importance to support the thesis goals. For this reason the uncertainty in the loss measurements is not discussed. Three different cases are discussed that may have an effect on the accuracy of measurements. The first case is if the measured and simulated $f_{0}$ of the cavity differ. The second case is if the variations in substrate properties are ignored. The last case is for uncertainty in the thickness of the MUT.

If the measured and simulated $f_{\mathrm{o}}$ of the cavity differ, there will be an increase in the uncertainty of the dielectric constant of the MUT. However, the added uncertainty is minimal. The cavity was simulated with two different values for the DR(35 and 35.27) and two different thicknesses for the glass cover slip(130 $\mu m$ and $160 \mu m)$. Next, the same simulations were performed with the addition of a $10 \mu \mathrm{m}$ thick material and the dielectric constant of the material was swept from 4-8. Using these results, the shift in $f_{0}$ was graphed and proved to be almost identical for all simulations.

Assuming all the glass cover slips have exactly the same properties in simulation (thickness and permittivity) affects the accuracy of properties of the MUT. The results 
from simulating for the shift in $f_{\mathrm{o}}$ with different glass substrates is shown in Figure 5.13. This demonstrates that even relatively large differences between glass cover slips will have a minimal effect on the accuracy of $\epsilon_{r}^{\prime}$ measurements.

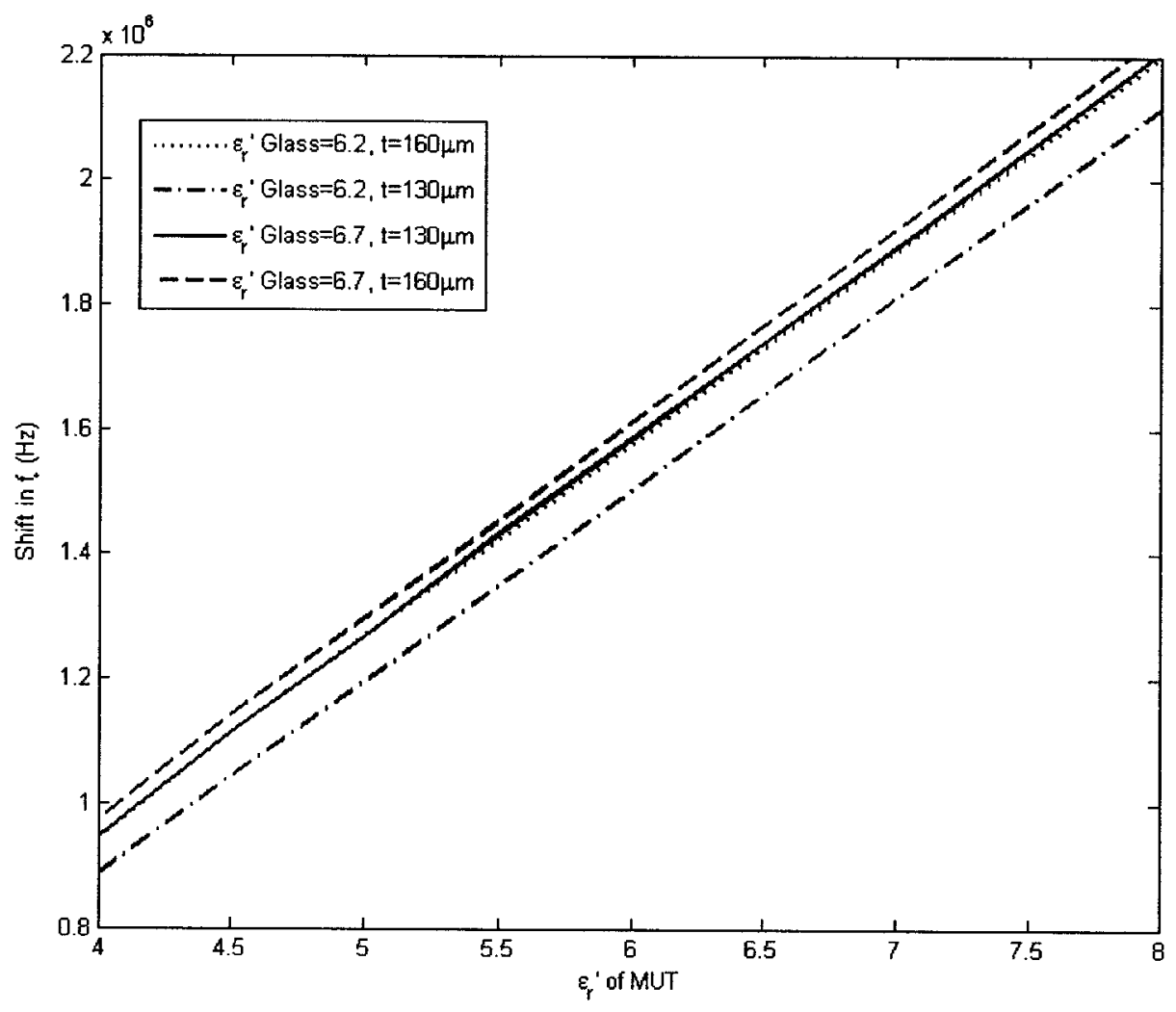

Figure 5.13: Effect of variances in the properties of the glass substrates on the shift in $f_{0}$ caused by the MUT

Uncertainty in the thickness of the MUT will cause uncertainty in the measurement of $\epsilon_{r}^{\prime}$, and is shown in Figure 5.14 by the differences in $f_{\mathrm{o}}$. 


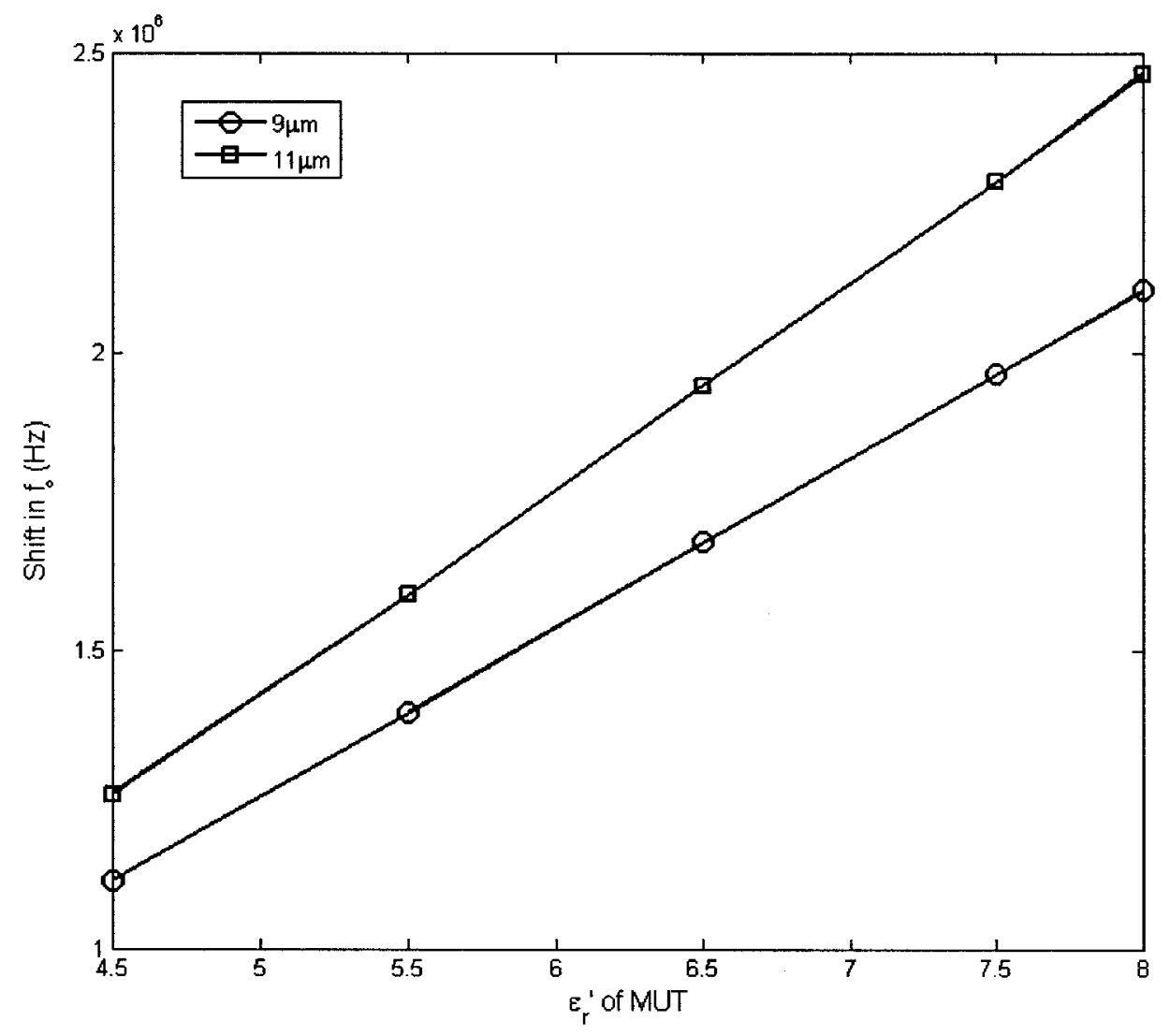

Figure 5.14: Effect of uncertainty in thickness of MUT

The SPDR is very similar to the material characterization apparatus describes in this work. For the SPDR the main source of uncertainty when measuring the dielectric constant of materials is the uncertainty in thickness of the MUT and is given by Equation 5.1 below [ ]

$$
\frac{\Delta \epsilon_{r}^{\prime}}{\epsilon_{r}^{\prime}}=2 \frac{\Delta t}{t}
$$

The uncertainty in the thickness of the MUT is the largest contributor to the 
uncertainty in $\epsilon_{r}^{\prime}$, however by accurately measuring the material thickness the uncertainty in the measurement of material properties can be controlled. Knowing the absolute permittivity would be useful, but it is not absolutely necessary for this work. It is expected that the effective dielectric constant of the material will increase upon illumination. This leads to the question of how accurate is this relative shift upon illumination. All the figures have graphs that are linear and almost parallel to each other. This suggests that the relative change in the $\epsilon_{r}^{\prime}$ upon illumination is more accurate than the absolute value of $\epsilon_{r}^{\prime}$.

\subsection{Tuning and Verification of Cavity}

The brass cavity for the OSiPDR was fabricated at the Carleton University Science Technology Centre. After all the components of the OSiPDR were assembled it was necessary to test the apparatus. The apparatus is shown in Figures 5.15 and 5.16. Following initial tests of the apparatus, the properties of the DR used in simulation were adjusted in order to ensure the measured and simulated results matched. Next, the empty cavity was analyzed at different temperatures and with or without illumination. Finally, the properties of the glass cover slips that were used, were measured and compared with the low frequency values.

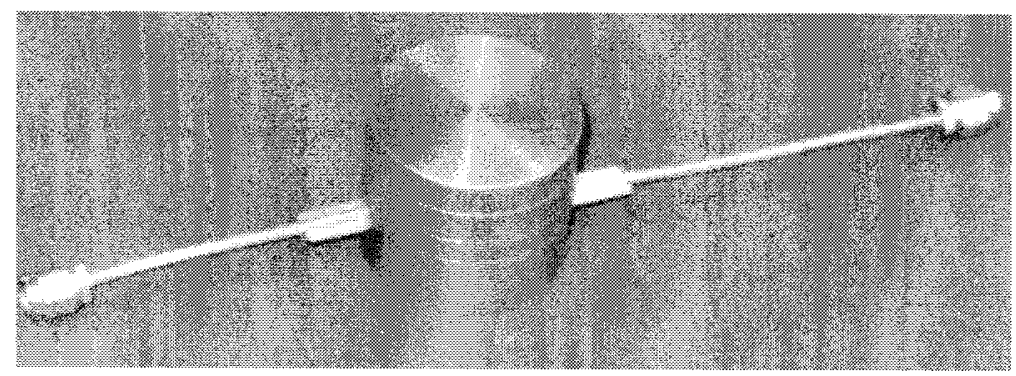

Figure 5.15: Full measurement apparatus (diameter of apparatus is $35 \mathrm{~mm}$ ) 


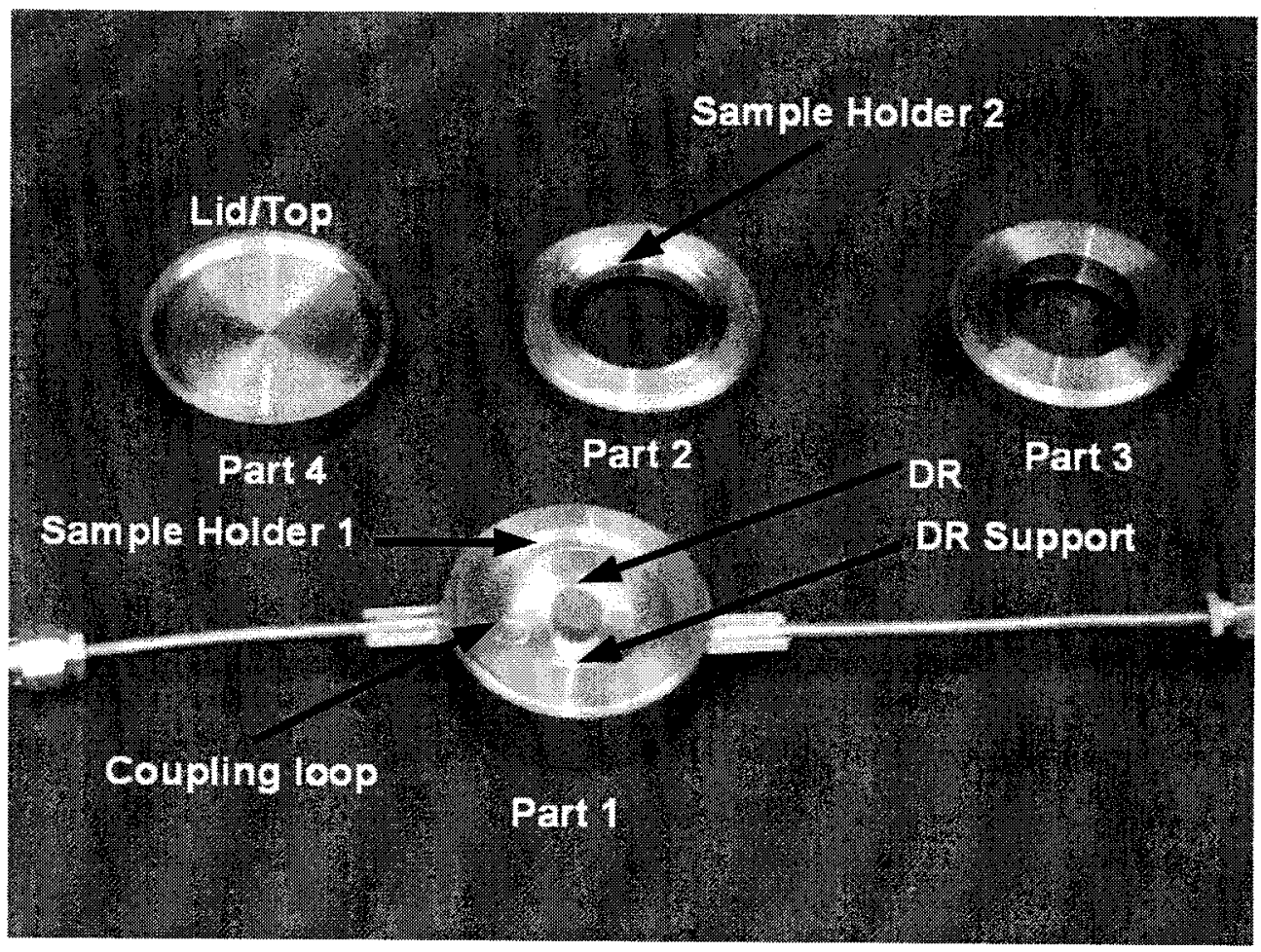

Figure 5.16: Parts of Measurement apparatus

\subsubsection{Adjustment of Simulation Parameters}

The components used in the OSiPDR had uncertainty in their properties. This made it necessary to match some of the simulation parameters to the measured results. The resonant cavity was measured with an Agilent $8720 \mathrm{ES}$ vector network analyzer (VNA) calibrated with an Agilent $85052 \mathrm{D} 3.5 \mathrm{~mm}$ economy calibration kit. The OSiPDR was connected to the VNA and the $f_{\circ}$ and $Q_{\circ}$ were extracted using the theory given in section 4.7. Using the value of the $f_{0}$ the dielectric constant of the DR in the simulation was adjusted until the $f_{0}$ matched the measured $f_{0}$. The dielectric constant of the DR was determined to be 35.27 , which fits within the range of uncertainty provided by the manufacturer of $35.5 \pm 1$. The $Q_{\circ}$ of the cavity was a slightly more difficult parameter to match with the simulated results. This was 
because the measured $Q_{0}$ is affected by variations in humidity, which could not be kept constant with the setup used. This meant that the most accurate $Q_{0}$ was the highest $Q_{0}$ measured, and this was the value used. The highest $Q_{\circ}$ measured was 5249 , this gave the loss tangent of the DR to be 0.000185 . These changes did not make the simulation match the experimental results perfectly because it was assumed all the differences were due to the DR. However, this was the best assumption that could be made because variations in the $\mathrm{DR}$ had the greatest effect on properties of the cavity.

\subsubsection{Analysis of Empty Cavity}

One of the initial measurements made on the cavity was to confirm that having the cavity open to the air had little effect on performance. These measurements were made with the cavity connected to the VNA (12.5KHz frequency step size) and the results $\left(f_{\circ}\right.$ and $Q_{\circ}$ which were obtained from reflection measurements) with and without the lid are shown in Table 5.2. These results show that the $f_{\circ}$ and the $Q_{\circ}$ decrease with the lid off. The change was small and resulted in minimal decrease in the accuracy of measurements.

Table 5.2: Characteristics of cavity with and without the lid

\begin{tabular}{|c|l|c|}
\hline Lid On & $f_{O}(\mathrm{GHz})$ & $Q_{O}$ \\
\hline $\mathrm{Y}$ & 7.9202375000 & 5168 \\
\hline $\mathrm{Y}$ & 7.9202125000 & 5168 \\
\hline $\mathrm{Y}$ & 7.9202500000 & 5195 \\
\hline $\mathrm{N}$ & 7.9201250000 & 5122 \\
\hline $\mathrm{N}$ & 7.9201250000 & 5136 \\
\hline $\mathrm{N}$ & 7.9201250000 & 5161 \\
\hline
\end{tabular}

Next, understanding how the resonant cavity responds to changes in temperature, humidity, and light was important so that measurements are interpreted properly. 
The setup shown in Figure 5.17 was used to characterize the empty cavity.

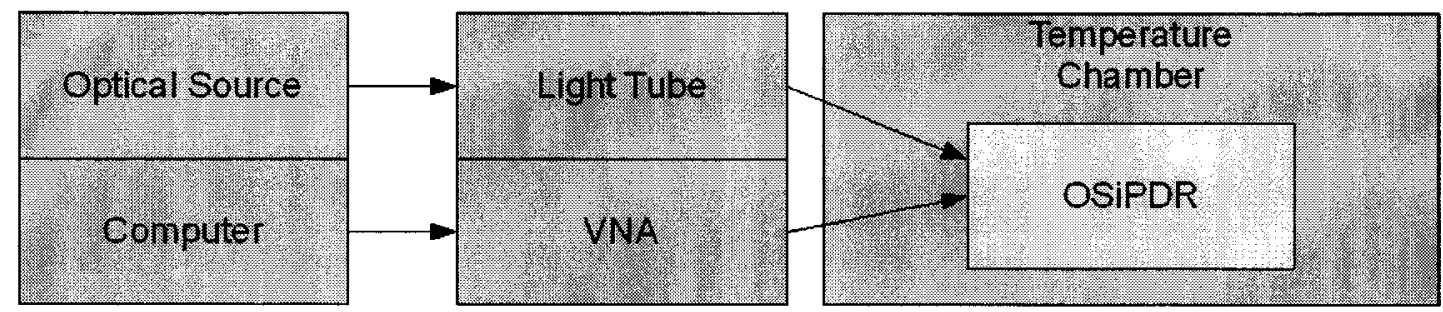

Figure 5.17: Setup used to measure properties of OSiPDR

Initially measurements were to be made at lower temperatures, however as the temperature was lowered the $Q_{0}$ of the OSiPDR decreased significantly. When the cavity was inspected, condensed water was seen on the OSiPDR. This meant that measurements below room temperature would yield poor results and were not made. The procedure for measuring the empty cavity was as follows:

1. Set temperature of the chamber to $30^{\circ} \mathrm{C}$

2. Once $30^{\circ} \mathrm{C}$ is reached, wait for at least 5 minutes before making measurements

3. Measure s-parameters of cavity without light

4. Measure s-parameters of cavity with light

5. Repeat the previous two steps at least two more times

6. Repeat above steps at $40^{\circ} \mathrm{C}$ and $50^{\circ} \mathrm{C}$

The results from measuring the empty cavity are shown in Table 5.3 
Table 5.3: Characteristics of empty cavity

\begin{tabular}{|c|c|l|l|}
\hline Temperature ${ }^{\circ} \mathrm{C}$ & Light On & $f_{o}(\mathrm{GHz})$ & $Q_{o}$ \\
\hline 30 & $\mathrm{~N}$ & 7.9201125000 & 5014.28 \\
\hline 30 & $\mathrm{~N}$ & 7.9201625000 & 5064.64 \\
\hline 30 & $\mathrm{~N}$ & 7.9201375000 & 5059.48 \\
\hline 30 & $\mathrm{~N}$ & 7.9201500000 & 5045.47 \\
\hline 30 & $\mathrm{Y}$ & 7.9201375000 & 5018.20 \\
\hline 30 & $\mathrm{Y}$ & 7.9201750000 & 5062.75 \\
\hline 30 & $\mathrm{Y}$ & 7.9201375000 & 5055.94 \\
\hline 30 & $\mathrm{Y}$ & 7.9201750000 & 5059.86 \\
\hline 40 & $\mathrm{~N}$ & 7.9202750000 & 5004.12 \\
\hline 40 & $\mathrm{~N}$ & 7.9202625000 & 5001.25 \\
\hline 40 & $\mathrm{~N}$ & 7.9202750000 & 5001.51 \\
\hline 40 & $\mathrm{Y}$ & 7.9202625000 & 5017.36 \\
\hline 40 & $\mathrm{Y}$ & 7.9202875000 & 4999.74 \\
\hline 40 & $\mathrm{Y}$ & 7.9202875000 & 4983.65 \\
\hline 50 & $\mathrm{~N}$ & 7.9203375000 & 4932.07 \\
\hline 50 & $\mathrm{~N}$ & 7.9203625000 & 4912.61 \\
\hline 50 & $\mathrm{~N}$ & 7.9203500000 & 4911.86 \\
\hline 50 & $\mathrm{Y}$ & 7.9203375000 & 4928.09 \\
\hline 50 & $\mathrm{Y}$ & 7.9203375000 & 4927.43 \\
\hline 50 & $\mathrm{Y}$ & 7.9203500000 & 4926.56 \\
\hline
\end{tabular}

From the data presented above one can see that the $f_{\circ}$ of the cavity increased as the temperature increased. The increase in resonant frequency meant that the dielectric constant of the DR decreased with an increase in temperature. Furthermore, one can see that the $Q_{0}$ of the cavity decreased with an increase in temperature, which meant the loss of the DR increases with temperature. These results match theory on the dielectric properties of materials. The theory states that increasing temperature usually increases the disorder in the material which prevents dipole moments from staying aligned with the applied electric field, and thus increases the dielectric loss tangent and decreased the dielectric constant. The light did not seem to affect the properties of the OSiPDR significantly, which meant that the light was not heating up the DR. From Table 5.3 one can see there was a variation in $f_{\circ}$ at each temperature point. The variation in $f_{\mathrm{o}}$ could be due to measurement error and uncertainty in temperature. The value of $Q_{0}$ was not stable at any of the temperature points. This 
could be due to measurement error, uncertainty in temperature, and changes in humidity. The results presented in Table 5.3 support that the OSiPDR was functioning as expected.

\subsubsection{OSiPDR with Glass Substrates}

The next step was to confirm material properties could be extracted using the OSiPDR. To accomplish this two different sets of measurements were made on glass substrates. One set of measurements was made on all the glass cover slips before any material was deposited. This set was made at room temperature and with a step size between frequency points of $187.5 \mathrm{kHz}$. The second set of measurements was made using the same setup and measurement procedure as that used to measure the empty cavity (resolution of $6.25 \mathrm{kHz}$ for $f_{\circ}$ measurements).

The results from measuring the glass substrates before samples were deposited is shown in Table 5.4. The results from measuring the glass cover slips over a range of temperature and with and without light are shown in Tables 5.5-5.7. To determine the shift in $f_{\mathrm{o}}$ for the results in Table 5.4 the $f_{\mathrm{o}}$ for the empty cavity listed in the Table 5.4 was used. To determine the shift in $f_{\circ}$ for Tables 5.5-5.7 the $f_{\circ}$ values of the empty cavity shown in Table 5.3 were used. More specifically, the value of $f_{\circ}$ used for determining the shift in $f_{0}$ was the first value recorded at each temperature without light. The decrease in the $Q_{0}$ with temperature was not accounted for at each temperature point. Instead the $Q_{\circ}$ value of 5249 was used for the empty cavity and the effects of humidity were calculated out using the results from Table 5.3. The results presented in Tables 5.4-5.7 are also shown in Figures 5.18-5.21. The error bars (thin lines) in Figures 5.18-5.21 represent the glass cover slips going from $160 \mu \mathrm{m}$ to $130 \mu \mathrm{m}$. The glass substrates that had the optically tunable dielectric material deposited on them were labeled a1-a20 (not all were used), and the additional glass 
cover slips were labeled S1-S3.

Table 5.4: Characteristics of substrates before material depositing ( $\mathrm{t}$ is the thickness of the glass)

\begin{tabular}{|c|l|l|c|c|c|c|}
\hline Sample & $f_{O}(\mathrm{GHz})$ & $Q_{0}$ & $\epsilon_{r}{ }^{\prime}, t=130 \mu m$ & $\epsilon_{r}{ }^{\prime}, t=160 \mu m$ & $\tan \delta, t=130 \mu m$ & $\tan \delta . t=160 \mu m$ \\
\hline Empty Cavity & 7.9201873920 & 4848 & - & - & - & - \\
\hline a1 & 7.8933749760 & 3231 & 7.2 & 6.1 & 0.013 & 0.013 \\
\hline a2 & 7.8935623680 & 3374 & 7.1 & 6.1 & 0.012 & 0.011 \\
\hline a3 & 7.8935623680 & 3375 & 7.1 & 6.1 & 0.012 & 0.011 \\
\hline a4 & 7.8930001920 & 3361 & 7.3 & 6.2 & 0.011 & 0.011 \\
\hline a5 & 7.8935623680 & 3229 & 7.1 & 6.1 & 0.013 & 0.013 \\
\hline a6 & 7.8926248960 & 3207 & 7.4 & 6.2 & 0.013 & 0.013 \\
\hline a7 & 7.8933749760 & 3355 & 7.2 & 6.1 & 0.012 & 0.011 \\
\hline a8 & 7.8931875840 & 3350 & 7.2 & 6.1 & 0.012 & 0.011 \\
\hline a9 & 7.8931875840 & 3363 & 7.2 & 6.1 & 0.012 & 0.011 \\
\hline a10 & 7.8937497600 & 3385 & 7.1 & 6.0 & 0.011 & 0.011 \\
\hline a11 & 7.8931875840 & 3365 & 7.2 & 6.1 & 0.011 & 0.011 \\
\hline a12 & 7.8918748160 & 3198 & 7.5 & 6.4 & 0.013 & 0.013 \\
\hline a13 & 7.8933749760 & 3368 & 7.2 & 6.1 & 0.012 & 0.011 \\
\hline a14 & 7.8935623680 & 3373 & 7.1 & 6.1 & 0.012 & 0.011 \\
\hline a15 & 7.8933749760 & 3372 & 7.2 & 6.1 & 0.011 & 0.011 \\
\hline a16 & 7.8930001920 & 3366 & 7.3 & 6.2 & 0.011 & 0.011 \\
\hline a17 & 7.8930001920 & 3369 & 7.3 & 6.2 & 0.011 & 0.011 \\
\hline a18 & 7.8930001920 & 3358 & 7.3 & 6.2 & 0.011 & 0.011 \\
\hline a19 & 7.8930001920 & 3224 & 7.3 & 6.2 & 0.013 & 0.013 \\
\hline a20 & 7.8931875840 & 3366 & 7.2 & 6.1 & 0.011 & 0.011 \\
\hline
\end{tabular}

Table 5.5: Characteristics glass cover slip S1 ( $\mathrm{t}$ is the thickness of the glass)

\begin{tabular}{|c|c|c|c|c|c|c|c|}
\hline Temp ${ }^{\circ} \mathrm{C}$ & Light & $f_{0}(\mathrm{GHz})$ & $\mathrm{g}_{0}$ & $\epsilon_{r}{ }^{\prime}, t=130 \mu m$ & $\epsilon_{r}{ }^{\prime}, t=160 \mu m$ & $\tan \delta, t=130 \mu m$ & $\tan \delta, t=160 \mu m$ \\
\hline 30 & $\mathrm{~N}$ & 7.8909125000 & 3422 & 7.7 & 6.5 & 0.011 & 0.011 \\
\hline 30 & $\mathrm{~N}$ & 7.8909250000 & 3430 & 7.7 & 6.5 & 0.011 & 0.011 \\
\hline 30 & $\mathrm{~N}$ & 7.8909250000 & 3421 & 7.7 & 6.5 & 0.011 & 0.011 \\
\hline 30 & $\mathrm{Y}$ & 7.8909250000 & 3420 & 7.7 & 6.5 & 0.011 & 0.011 \\
\hline 30 & $\mathrm{Y}$ & 7.8909250000 & 3420 & 7.7 & 6.5 & 0.011 & 0.011 \\
\hline 30 & $\mathrm{Y}$ & 7.8909250000 & 3420 & 7.7 & 6.5 & 0.011 & 0.011 \\
\hline 40 & $\mathrm{~N}$ & 7.8909250000 & 3380 & 7.8 & 6.6 & 0.011 & 0.011 \\
\hline 40 & $\mathrm{~N}$ & 7.8909250000 & 3388 & 7.8 & 6.6 & 0.011 & 0.011 \\
\hline 40 & $\mathrm{~N}$ & 7.8909250000 & 3388 & 7.8 & 6.6 & 0.011 & 0.011 \\
\hline 40 & $\mathrm{Y}$ & 7.8909375000 & 3388 & 7.8 & 6.6 & 0.011 & 0.011 \\
\hline 40 & $\mathrm{Y}$ & 7.8909250000 & 3387 & 7.8 & 6.6 & 0.011 & 0.011 \\
\hline 40 & $\mathrm{Y}$ & 7.8909500000 & 3378 & 7.8 & 6.6 & 0.011 & 0.011 \\
\hline 50 & $\mathrm{~N}$ & 7.8909250000 & 3327 & 7.8 & 6.6 & 0.012 & 0.012 \\
\hline 50 & $\mathrm{~N}$ & 7.8909250000 & 3325 & 7.8 & 6.6 & 0.012 & 0.012 \\
\hline 50 & $\mathrm{~N}$ & 7.3909250000 & 3324 & 7.8 & 6.6 & 0.012 & 0.012 \\
\hline 50 & $\mathrm{Y}$ & 7.8909375000 & 3316 & 7.8 & 6.6 & 0.012 & 0.012 \\
\hline 50 & $\mathrm{Y}$ & 7.8909375000 & 3315 & 7.8 & 6.6 & 0.012 & 0.012 \\
\hline 50 & $\mathrm{Y}$ & 7.3909250000 & 3314 & 7.8 & 6.6 & 0.012 & 0.012 \\
\hline
\end{tabular}


Table 5.6: Characteristics glass cover slip S2 ( $\mathrm{t}$ is the thickness of the glass)

\begin{tabular}{|c|c|c|c|c|c|c|c|}
\hline Temp ${ }^{\circ} \mathrm{C}$ & Light & $f_{\circ}(\mathrm{GHZ})$ & $\mathrm{Q}_{o}$ & $\epsilon_{r}^{\prime}, t=130 \mu m$ & $\left.\epsilon_{r}^{\prime}, t=16\right) \mu m$ & $\tan \delta . t=130 \mu m$ & $\tan \delta . t=160 \mu m$ \\
\hline 30 & $\mathrm{~N}$ & 7.8927000000 & 3452 & 7.3 & 6.2 & 0.011 & 0.011 \\
\hline 30 & $\mathrm{~N}$ & 7.8927000000 & 3443 & 7.3 & 6.2 & 0.011 & 0.011 \\
\hline 30 & $\mathrm{~N}$ & 7.8927000000 & 3462 & 7.3 & 6.2 & 0.011 & 0.011 \\
\hline 30 & $\mathrm{Y}$ & 7.8927125000 & 3452 & 7.3 & 6.2 & 0.011 & 0.011 \\
\hline 30 & $\mathrm{Y}$ & 7.3927125000 & 3452 & 7.3 & 6.2 & 0.011 & 0.011 \\
\hline 30 & $\mathrm{Y}$ & 7.8927125000 & 3462 & 7.3 & 6.2 & 0.011 & 0.011 \\
\hline 40 & $\mathrm{~N}$ & 7.8927250000 & 3432 & 7.4 & 6.2 & 0.011 & 0.011 \\
\hline 40 & $\mathrm{~N}$ & 7.8927375000 & 3440 & 7.3 & 6.2 & 0.011 & 0.011 \\
\hline 40 & $\mathrm{~N}$ & 7.8927375000 & 3430 & 7.3 & 6.2 & 0.011 & 0.011 \\
\hline 40 & $\mathrm{Y}$ & 7.8927375000 & 3430 & 7.3 & 6.2 & 0.011 & 0.011 \\
\hline 40 & $\mathrm{Y}$ & 7.8927375000 & 3430 & 7.3 & 6.2 & 0.011 & 0.011 \\
\hline 40 & $\mathrm{Y}$ & 7.8927375000 & 3429 & 7.3 & 6.2 & 0.011 & 0.011 \\
\hline 50 & $\mathrm{~N}$ & 7.8927250000 & 3367 & 7.4 & 6.2 & 0.012 & 0.012 \\
\hline 50 & $\mathrm{~N}$ & 7.8927250000 & 3356 & 7.4 & 6.2 & 0.012 & 0.012 \\
\hline 50 & $\mathrm{~N}$ & 7.8927250000 & 3355 & 7.4 & 6.2 & 0.012 & 0.012 \\
\hline 50 & $\mathrm{Y}$ & 7.8927375000 & 3365 & 7.4 & 6.2 & 0.012 & 0.012 \\
\hline 50 & $\mathrm{Y}$ & 7.8927250000 & 3364 & 7.4 & 6.2 & 0.012 & 0.012 \\
\hline 50 & $\mathrm{Y}$ & 7.8927250000 & 3355 & 7.4 & 6.2 & 0.012 & 0.012 \\
\hline
\end{tabular}

Table 5.7: Characteristics glass cover slip S3 ( $t$ is the thickness of the glass)

\begin{tabular}{|c|c|c|c|c|c|c|c|}
\hline Temp ${ }^{\circ} \mathrm{C}$ & Light & $f_{\circ}(\mathrm{GHz})$ & $Q_{0}$ & $\epsilon_{r}{ }^{\prime}, t=130 \mu m$ & $\epsilon_{r}{ }^{\prime}, t=160 \mu m$ & $\tan \delta, t=130 \mu m$ & $\tan \delta . t=100 \mu m$ \\
\hline 30 & $\mathrm{~N}$ & 7.8927250000 & 3548 & 7.3 & 6.2 & 0.010 & 0.010 \\
\hline 30 & $\mathrm{~N}$ & 7.8933000000 & 3536 & 7.2 & 6.1 & 0.011 & 0.010 \\
\hline 30 & $\mathrm{~N}$ & 7.8933000000 & 3536 & 7.2 & 6.1 & 0.011 & 0.010 \\
\hline 30 & $\mathrm{Y}$ & 7.8933000000 & 3536 & 7.2 & 6.1 & 0.011 & 0.010 \\
\hline 30 & $\mathrm{Y}$ & 7.8933125000 & 3535 & 7.2 & 6.1 & 0.011 & 0.010 \\
\hline 30 & $\mathrm{Y}$ & 7.8933000000 & 3535 & 7.2 & 6.1 & 0.011 & 0.010 \\
\hline 40 & $\mathrm{~N}$ & 7.8932875000 & 3482 & 7.2 & 6.1 & 0.011 & 0.011 \\
\hline 40 & $\mathrm{~N}$ & 7.8932750000 & 3469 & 7.2 & 6.1 & 0.011 & 0.011 \\
\hline 40 & $\mathrm{~N}$ & 7.8932750000 & 3477 & 7.2 & 6.1 & 0.011 & 0.011 \\
\hline 40 & $\mathrm{Y}$ & 7.8932750000 & 3479 & 7.2 & 6.1 & 0.011 & 0.011 \\
\hline 40 & $\mathrm{Y}$ & 7.8932875000 & 3477 & 7.2 & 6.1 & 0.011 & 0.011 \\
\hline 40 & $\mathrm{Y}$ & 7.8932875000 & 3475 & 7.2 & 6.1 & 0.011 & 0.011 \\
\hline 50 & $\mathrm{~N}$ & 7.8932625000 & 3424 & 7.2 & 6.1 & 0.012 & 0.011 \\
\hline 50 & $\mathrm{~N}$ & 7.8932750000 & 3412 & 7.2 & 6.1 & 0.012 & 0.011 \\
\hline 50 & $\mathrm{~N}$ & 7.8932875000 & 3411 & 7.2 & 6.1 & 0.012 & 0.011 \\
\hline 50 & $\mathrm{Y}$ & 7.8932875000 & 3412 & 7.2 & 6.1 & 0.012 & 0.011 \\
\hline 50 & $\mathrm{Y}$ & 7.8932875000 & 3411 & 7.2 & 6.1 & 0.012 & 0.011 \\
\hline 50 & $\mathrm{Y}$ & 7.8932875000 & 3401 & 7.2 & 6.1 & 0.012 & 0.012 \\
\hline
\end{tabular}




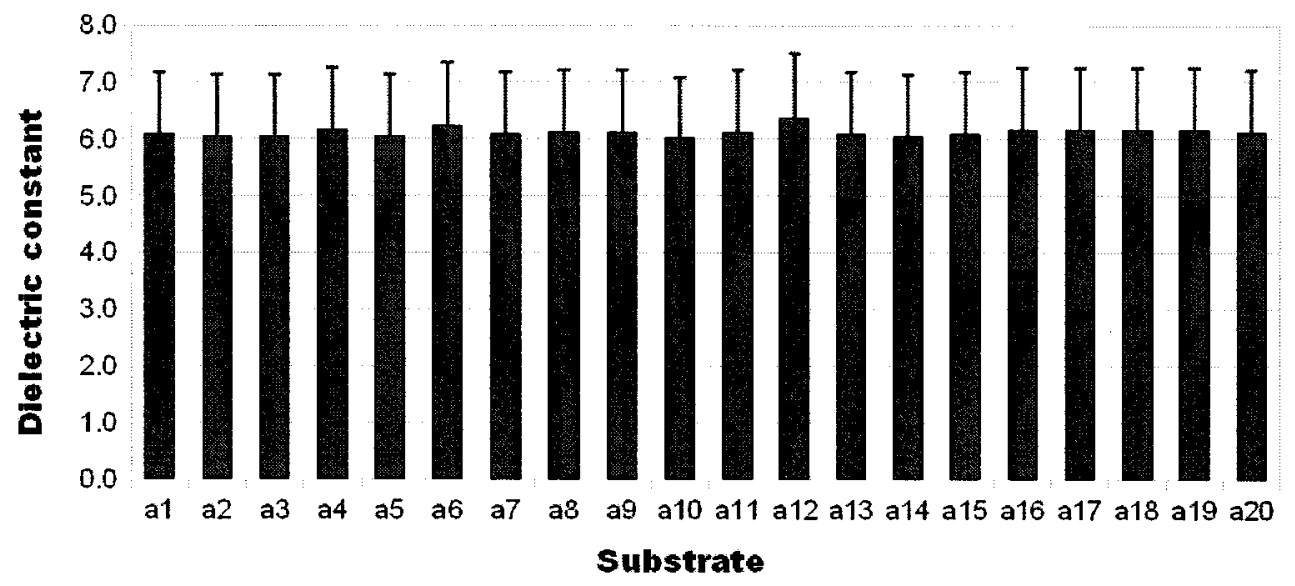

Figure 5.18: Dielectric constant of substrates before material is deposited on them $(t=160 \mu m)$

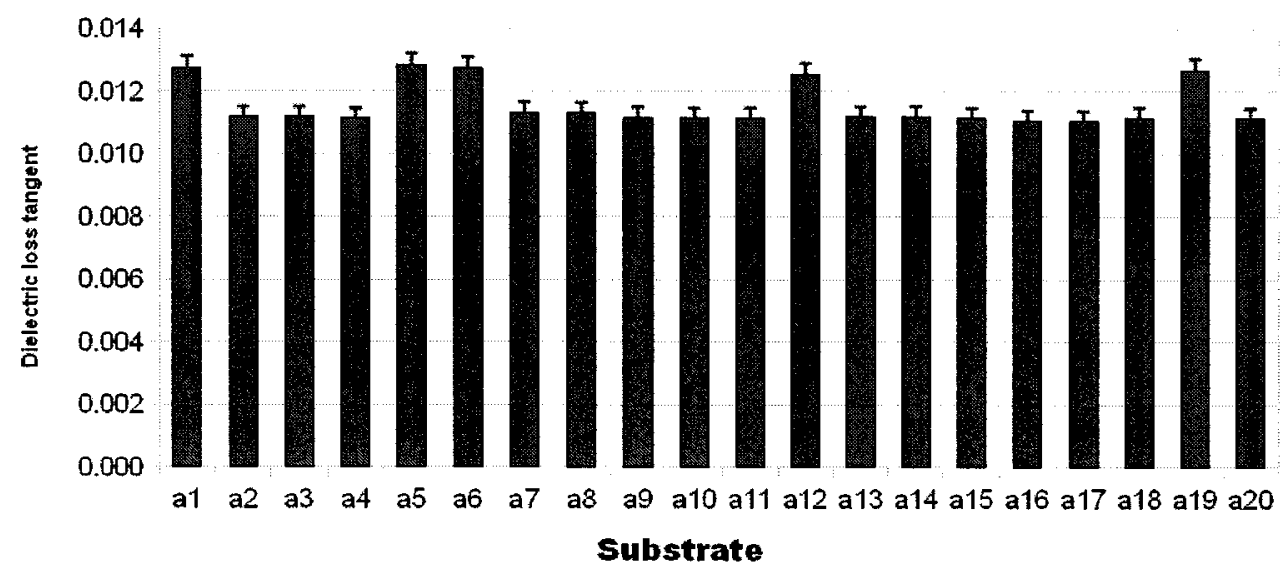

Figure 5.19: Dielectric loss of substrates before material is deposited on them $(t=$ $160 \mu \mathrm{m})$ 


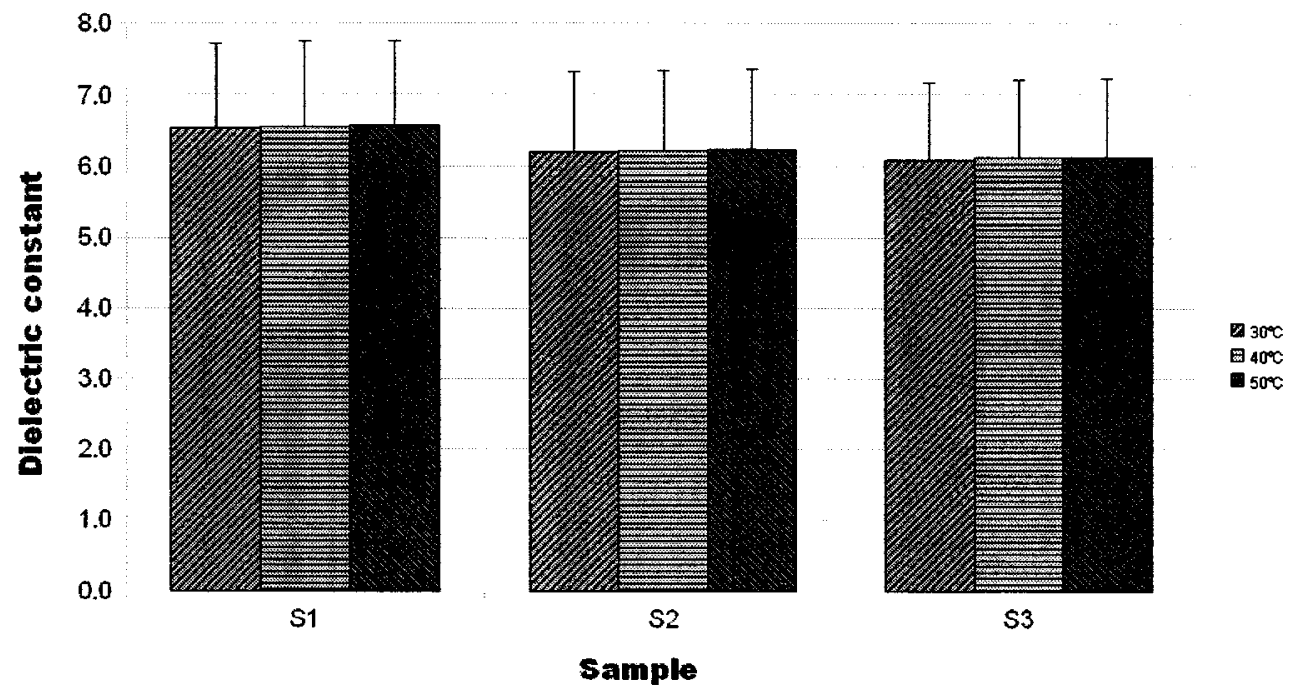

Figure 5.20: Dielectric constant of glass cover slips $(t=160 \mu m)$

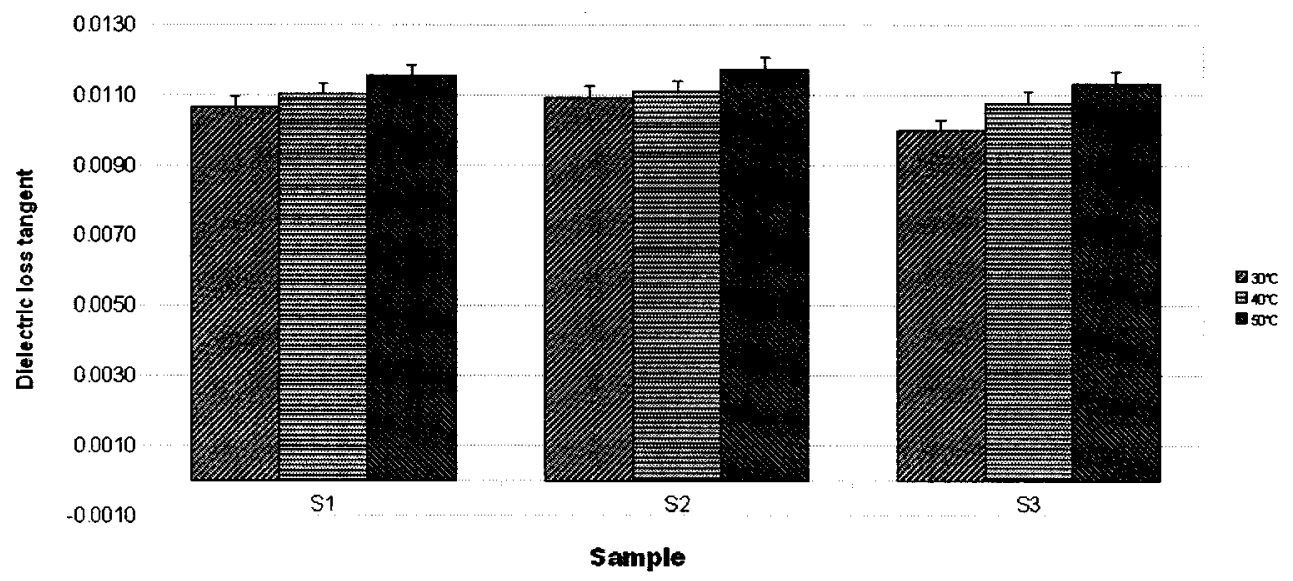

Figure 5.21: Dielectric loss of glass cover slips $(t=160 \mu \mathrm{m})$

The thickness of the glass cover slips could not be measured to a higher degree of accuracy than the manufacturer provided. For this reason all the data are analyzed for the thickness of the cover slips being between $130-160 \mu \mathrm{m}$. The results show that the dielectric constants of the glass is between 6.1 and 7.8. However, this range in dielectric constants can be minimized further. From Tables 5.4-5.7 one can see that the range from 6.5-7.1 are the only dielectric constant that overlap for all samples. It 
is not expected that the dielectric constant of the glass is higher than the value given by the manufacturer at $1 \mathrm{MHz}$ of 6.7 . Also, the loss tangent of the glass is greater than the 0.0046 measured at $1 \mathrm{MHz}$. An increase in loss is usually accompanied by a decrease in the dielectric constant. For these reasons the dielectric constant of the glass should be between 6.5 and 6.7 . These results show that the cavity can measure the properties of materials, however large uncertainty in thickness causes large uncertainty in dielectric constant.

The results in Tables 5.5-5.7 demonstrate that the application of light to the glass cover slips had no effect on the dielectric constant. Figure 5.20 shows an increase in temperature caused a slight increase in the dielectric constant of the glass cover slips. This result is accompanied by an increase in dielectric loss. However, this increase in loss appears to be the same amount as measured for the empty cavity in Table 5.3. The increase in the dielectric constant of the glass cover slips could potentially be problematic and must be accounted for when material properties of samples placed on the glass are measured.

\subsection{Conclusions}

The material characterization apparatus designed and tested in this chapter functioned within expectations. The properties of the fabricated resonant cavity were very similar to the simulated results, and simulation values only required minor adjustment. The OSiPDR allowed for the properties of the glass cover slips to be measured, and the results matched with the expected values. There are many possible sources of error when measuring the properties of material using the resonant cavity. However, with proper precautions the uncertainty in the measurement of material properties can be minimized. 


\section{Chapter 6}

\section{Experimental Material Characterization}

\subsection{Introduction}

This chapter presents the results from measuring the properties of polymers containing photoconductive particles. The first section discusses the preparation of the materials that were measured. The next section presents the measurements performed to extract the microwave properties of the materials. The last section presents the optical transmission measurements of the materials.

\subsection{Material Preparation}

The materials were prepared by Professor Steven McGarry and will not be discussed in depth, only a brief overview is given. There were four different films made: polymer; polymer and dopant; polymer, CdS, and dopant; and polymer and CdS. The four different samples were made because of the process used to make the polymer and doped CdS samples. First the dopant (20 $\mathrm{mg}$ of $\mathrm{CuCl}_{2}$ ) was mixed with the polymer and solvent $(4 \mathrm{~g}, 20 \% 3571$ dielectric polymer from DuPont). Next, the CdS was added to the mixture and this mixture was left for some time to allow the copper 
to diffuse into the CdS. The volume fill fraction of the CdS was $72 \%$. Each of the four different samples were deposited using a screen printing process onto glass cover slips. Then the samples were cured on a hotplate at $140^{\circ} \mathrm{C}$ for at least 30 minutes. The polymer-CdS samples are shown in Figure 6.1.

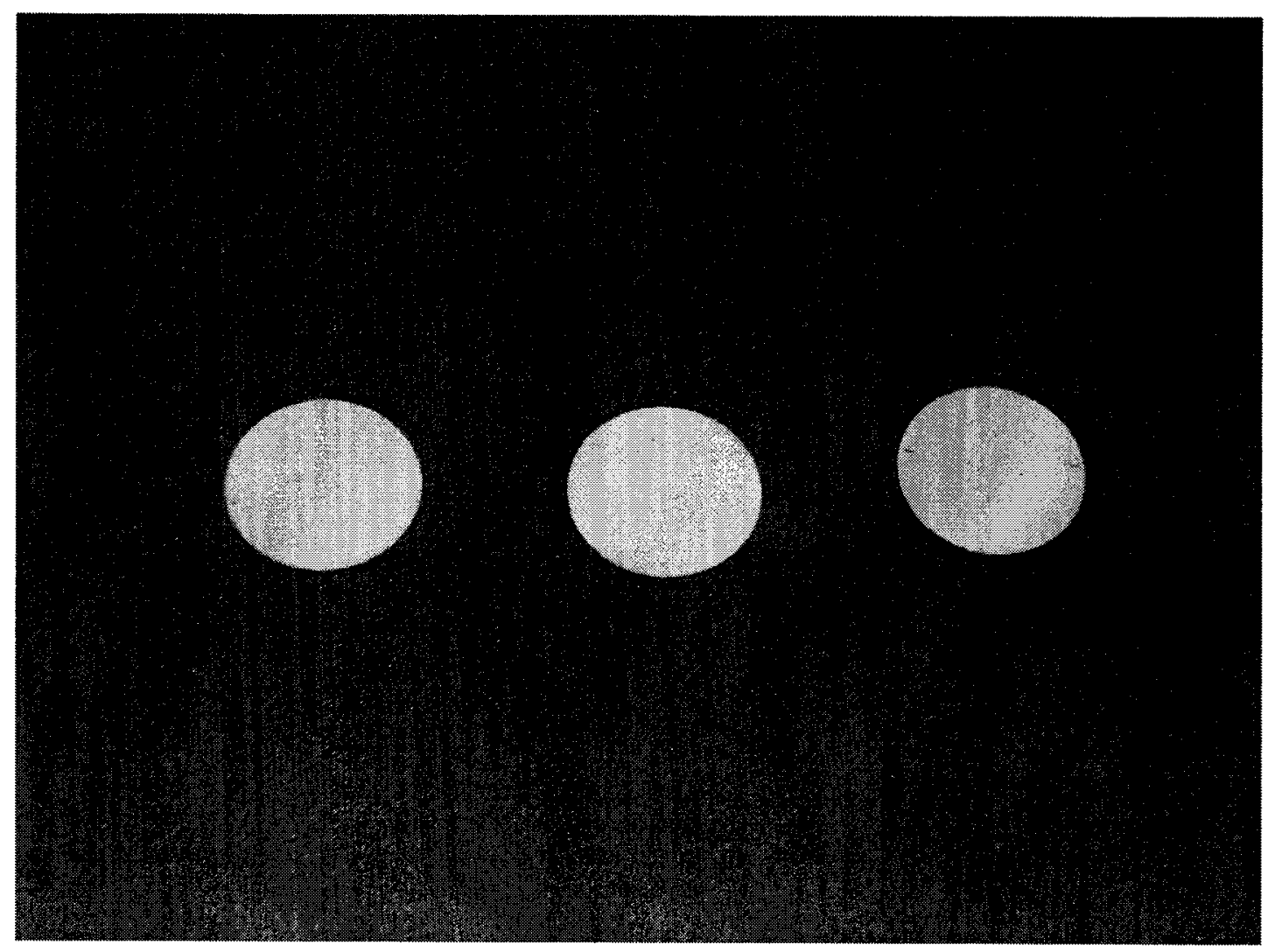

Figure 6.1: Glass cover slips with polymer-CdS deposited (scratch on leftmost one was made when measuring thickness)

Each of the samples were labeled using a diamond tipped scribe. The first sample of each type of material deposited (a3, a6, a9, and a12) had more imperfections than the second and third samples of each type. For this reason the results obtained from those samples are more suspect than other measurements.

The thickness of the samples were measured using a stylus profilometer Dektak. Measuring the polymer and polymer-dopant samples proved difficult due to the fact 
they were thinner than expected. The measurements yielded a thickness of $1.5-2 \mu \mathrm{m}$. However, this result was suspect because it was difficult to obtain in a repeatable manner and the samples were much thinner than expected. The polymer and polymerdopant samples were measured with the OSiPDR and the shift in resonant frequency was within the resolution of the measurement. This would have only happened if the samples were thinner than $1.5 \mu \mathrm{m}$. The results from the polymer and polymer-dopant samples are not shown because the materials were too thin to be measured. This was not a limitation of the OSiPDR, it was because the glass cover slips, upon which the samples were deposited, were originally measured with a resolution of $187.5 \mathrm{kHz}$. The shift in $f_{\circ}$ for the samples were around $60 \mathrm{kHz}$. Measurements with a resolution of $12.5 \mathrm{kHz}$ (smallest resolution) should have been made and would have allowed valid results to be extracted. The samples with CdS were measured to have a thickness of 9-11 $\mu \mathrm{m}$. These looked to have a rough surface and some visible gaps, which means the thickness measurements are suspect for these samples as well.

\subsection{Microwave Material Property Characteriza- tion}

The setup shown in Figure 5.17 was used to measure the microwave properties of the films. The light source used was an Oriel quartz tungsten lamp (bulb 6333), and the spectrum of the light is shown in Figure 6.2. The optical power that was applied to the OSiPDR was measured to be $0.22 \mathrm{~W}$, and was measured with a Coherent 1 D100 thermopile sensor connected to a Coherent Lasermate 1D power meter. 


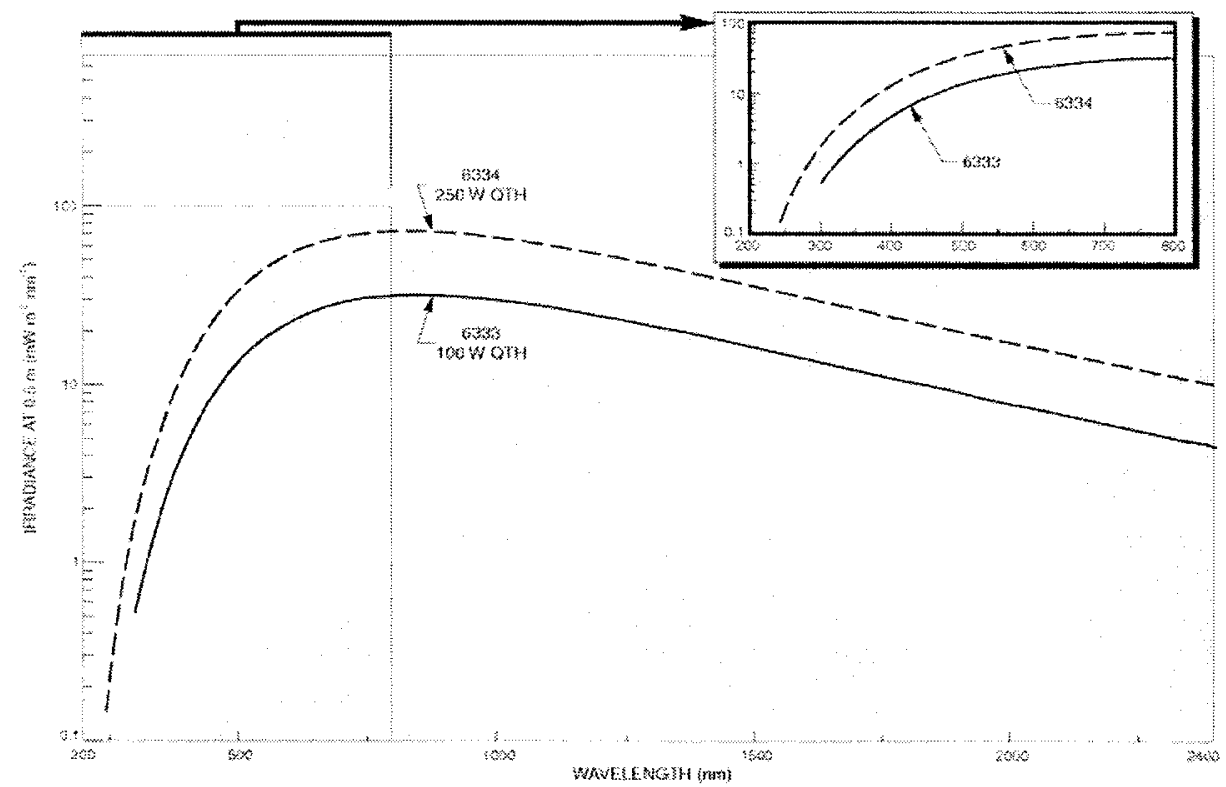

Figure 6.2: Spectrum of the lamp used (curve for 6333 is the bulb used) [

The procedure for measuring each sample was as follows:

1. Place sample in characterization apparatus

2. Set temperature of the temperature chamber to $30^{\circ} \mathrm{C}$

3. Leave at $30^{\circ} \mathrm{C}$ for at least 5 minutes before making measurements

4. Measure s-parameters of cavity without light

5. Measure s-parameters of cavity with light

6. Repeat the previous two steps two more times

7. Repeat measurements at $40^{\circ} \mathrm{C}$ and $50^{\circ} \mathrm{C}$

The results from measuring the polymer and doped CdS are shown in Tables 6.1-6.3. The results from measuring the polymer and CdS are shown in Tables 6.46.6. The order the measurements were made differs from how they are shown below. 
The results are arranged with the three measurement without light then three measurements with light for each temperature point. The first dark measurement at each temperature point represents the first measurement made, while the first measurement with light at each temperature point represents the second measurements made. The delay between measurements at each temperature point was less than 10 seconds. Only the raw data are presented below, the data will be analyzed and discussed further in Chapter 7.

Table 6.1: Results from measuring sample a9 (polymer and doped CdS)

\begin{tabular}{|c|c|c|c|c|c|c|c|}
\hline Temp ${ }^{\circ} \mathrm{C}$ & Light & $f_{0}(\mathrm{GHz})$ & $Q_{o}$ & $\epsilon_{r}^{\prime}, t=9 \mu m$ & $\epsilon_{r}^{\prime}, t=11 \mu m$ & $\tan \delta, t=9 \mu m$ & $\tan \delta, t=11 \mu m$ \\
\hline 30 & $\mathrm{~N}$ & 7.8923000000 & 3276 & 3.7 & 3.4 & 0.048 & 0.046 \\
\hline 30 & $\mathrm{~N}$ & 7.8922625000 & 3213 & 3.8 & 3.5 & 0.065 & 0.063 \\
\hline 30 & $\mathrm{~N}$ & 7.8922625000 & 3222 & 3.8 & 3.5 & 0.063 & 0.061 \\
\hline 30 & $\mathrm{Y}$ & 7.8921000000 & 2996 & 4.4 & 4.0 & 0.121 & 0.118 \\
\hline 30 & $\mathrm{Y}$ & 7.8921000000 & 2995 & 4.4 & 4.0 & 0.122 & 0.118 \\
\hline 30 & $\mathrm{Y}$ & 7.8921000000 & 2994 & 4.4 & 4.0 & 0.122 & 0.118 \\
\hline 40 & $\mathrm{~N}$ & 7.8922625000 & 3244 & 3.3 & 3.1 & 0.064 & 0.062 \\
\hline 40 & $\mathrm{~N}$ & 7.8922375000 & 3190 & 3.4 & 3.2 & 0.081 & 0.078 \\
\hline 40 & $\mathrm{~N}$ & 7.8922250000 & 3179 & 3.4 & 3.2 & 0.084 & 0.081 \\
\hline 40 & $\mathrm{Y}$ & 7.8920750000 & 2987 & 3.9 & 3.6 & 0.137 & 0.133 \\
\hline 40 & $\mathrm{Y}$ & 7.8920750000 & 2985 & 3.9 & 3.6 & 0.138 & 0.134 \\
\hline 40 & $\mathrm{Y}$ & 7.8920750000 & 2976 & 3.9 & 3.6 & 0.141 & 0.137 \\
\hline 50 & $\mathrm{~N}$ & 7.8922625000 & 3186 & 3.0 & 2.9 & 0.088 & 0.086 \\
\hline 50 & $\mathrm{~N}$ & 7.8922250000 & 3125 & 3.2 & 3.0 & 0.108 & 0.105 \\
\hline 50 & $\mathrm{~N}$ & 7.8922250000 & 3115 & 3.2 & 3.0 & 0.112 & 0.109 \\
\hline 50 & $\mathrm{Y}$ & 7.8920875000 & 2931 & 3.7 & 3.4 & 0.166 & 0.161 \\
\hline 50 & $\mathrm{Y}$ & 7.8920750000 & 2921 & 3.7 & 3.4 & 0.168 & 0.163 \\
\hline 50 & $\mathrm{Y}$ & 7.8920625000 & 2919 & 3.8 & 3.5 & 0.167 & 0.162 \\
\hline
\end{tabular}


Table 6.2: Results from measuring sample a10 (polymer and doped CdS)

\begin{tabular}{|c|c|c|c|c|c|c|c|}
\hline Temp ${ }^{\circ} \mathrm{C}$ & Light & $f_{o}(\mathrm{GHz})$ & $Q_{o}$ & $\epsilon_{r}{ }^{\prime}, t=9 \mu m$ & $\epsilon_{r}{ }^{\prime}, t=11 \mu m$ & $\tan \delta, t=9 \mu m$ & $\tan \delta, t=11 \mu m$ \\
\hline 30 & $\mathrm{~N}$ & 7.8924250000 & 3309 & 5.3 & 4.7 & 0.033 & 0.032 \\
\hline 30 & $\mathrm{~N}$ & 7.8924000000 & 3260 & 5.3 & 4.8 & 0.043 & 0.042 \\
\hline 30 & $\mathrm{~N}$ & 7.8924000000 & 3269 & 5.3 & 4.8 & 0.041 & 0.040 \\
\hline 30 & $\mathrm{Y}$ & 7.8922750000 & 3094 & 5.8 & 5.1 & 0.076 & 0.074 \\
\hline 30 & $\mathrm{Y}$ & 7.8922750000 & 3086 & 5.8 & 5.1 & 0.078 & 0.076 \\
\hline 30 & $\mathrm{Y}$ & 7.8922750000 & 3087 & 5.8 & 5.1 & 0.078 & 0.076 \\
\hline 40 & $\mathrm{~N}$ & 7.8923625000 & 3248 & 4.9 & 4.4 & 0.049 & 0.048 \\
\hline 40 & $\mathrm{~N}$ & 7.8923625000 & 3238 & 4.9 & 4.4 & 0.052 & 0.050 \\
\hline 40 & $\mathrm{~N}$ & 7.8923625000 & 3237 & 4.9 & 4.4 & 0.052 & 0.051 \\
\hline 40 & $\mathrm{Y}$ & 7.8922375000 & 3069 & 5.3 & 4.8 & 0.088 & 0.086 \\
\hline 40 & $\mathrm{Y}$ & 7.8922375000 & 3068 & 5.3 & 4.8 & 0.089 & 0.086 \\
\hline 40 & $\mathrm{Y}$ & 7.8922375000 & 3060 & 5.3 & 4.8 & 0.091 & 0.088 \\
\hline 50 & $\mathrm{~N}$ & 7.8924000000 & 3228 & 4.5 & 4.1 & 0.058 & 0.056 \\
\hline 50 & $\mathrm{~N}$ & 7.8923750000 & 3190 & 4.6 & 4.2 & 0.067 & 0.065 \\
\hline 50 & $\mathrm{~N}$ & 7.8923750000 & 3179 & 4.6 & 4.2 & 0.070 & 0.068 \\
\hline 50 & $\mathrm{Y}$ & 7.8922500000 & 3032 & 5.1 & 4.6 & 0.102 & 0.100 \\
\hline 50 & $\mathrm{Y}$ & 7.8922500000 & 3021 & 5.1 & 4.6 & 0.105 & 0.102 \\
\hline 50 & $\mathrm{Y}$ & 7.8922500000 & 3022 & 5.1 & 4.6 & 0.105 & 0.102 \\
\hline
\end{tabular}

Table 6.3: Results from measuring sample all (polymer and doped CdS)

\begin{tabular}{|c|c|c|c|c|c|c|c|}
\hline Temp ${ }^{\circ} \mathrm{C}$ & Light & $f_{o}(\mathrm{GHz})$ & $Q_{o}$ & $\epsilon_{r}{ }^{\prime}, t=9 \mu m$ & $\epsilon_{r}{ }^{\prime}, t=11 \mu m$ & $\tan \delta, t=9 \mu m$ & $\tan \delta, t=11 \mu m$ \\
\hline 30 & $\mathrm{~N}$ & 7.8924125000 & 3334 & 3.3 & 3.1 & 0.034 & 0.033 \\
\hline 30 & $\mathrm{~N}$ & 7.8923750000 & 3288 & 3.4 & 3.2 & 0.047 & 0.046 \\
\hline 30 & $\mathrm{~N}$ & 7.8923750000 & 3277 & 3.4 & 3.2 & 0.051 & 0.050 \\
\hline 30 & $\mathrm{Y}$ & 7.8922500000 & 3105 & 3.9 & 3.6 & 0.099 & 0.096 \\
\hline 30 & $\mathrm{Y}$ & 7.8922500000 & 3111 & 3.9 & 3.6 & 0.097 & 0.094 \\
\hline 30 & $\mathrm{Y}$ & 7.8922500000 & 3110 & 3.9 & 3.6 & 0.098 & 0.095 \\
\hline 40 & $\mathrm{~N}$ & 7.8923875000 & 3270 & 2.8 & 2.7 & 0.061 & 0.060 \\
\hline 40 & $\mathrm{~N}$ & 7.8923750000 & 3232 & 2.9 & 2.8 & 0.075 & 0.073 \\
\hline 40 & $\mathrm{~N}$ & 7.8923750000 & 3231 & 2.9 & 2.8 & 0.076 & 0.074 \\
\hline 40 & $\mathrm{Y}$ & 7.8922375000 & 3073 & 3.4 & 3.2 & 0.123 & 0.120 \\
\hline 40 & $\mathrm{Y}$ & 7.8922375000 & 3063 & 3.4 & 3.2 & 0.127 & 0.123 \\
\hline 40 & $\mathrm{Y}$ & 7.8922500000 & 3062 & 3.3 & 3.1 & 0.129 & 0.125 \\
\hline 50 & $\mathrm{~N}$ & 7.8924000000 & 3227 & 2.6 & 2.5 & 0.084 & 0.082 \\
\hline 50 & $\mathrm{~N}$ & 7.8923750000 & 3180 & 2.7 & 2.6 & 0.101 & 0.099 \\
\hline 50 & $\mathrm{~N}$ & 7.8923750000 & 3188 & 2.7 & 2.6 & 0.097 & 0.096 \\
\hline 50 & $\mathrm{Y}$ & 7.8922500000 & 3031 & 3.1 & 2.9 & 0.148 & 0.144 \\
\hline 50 & $\mathrm{Y}$ & 7.8922500000 & 3023 & 3.1 & 2.9 & 0.152 & 0.148 \\
\hline 50 & $\mathrm{Y}$ & 7.8922500000 & 3029 & 3.1 & 2.9 & 0.149 & 0.145 \\
\hline
\end{tabular}


Table 6.4: Results from measuring sample a12 (polymer and undoped CdS)

\begin{tabular}{|c|c|c|c|c|c|c|c|}
\hline Temp ${ }^{\circ} \mathrm{C}$ & Light & $f_{o}(\mathrm{GHz})$ & $\underline{Q}_{o}$ & $\epsilon_{r}{ }^{\prime}, t=9 \mu m$ & $\epsilon_{r}{ }^{\prime}, t=11 \mu m$ & $\tan \delta, t=9 \mu m$ & $\tan \delta, t=11 \mu m$ \\
\hline 30 & $\mathrm{~N}$ & 7.8905750000 & 3169 & 5.2 & 4.6 & 0.024 & 0.023 \\
\hline 30 & $\mathrm{~N}$ & 7.8904750000 & 3039 & 5.5 & 4.9 & 0.054 & 0.052 \\
\hline 30 & $\mathrm{~N}$ & 7.8904750000 & 3015 & 5.5 & 4.9 & 0.060 & 0.058 \\
\hline 30 & $\mathrm{Y}$ & 7.8903250000 & 2843 & 6.0 & 5.4 & 0.097 & 0.095 \\
\hline 30 & $\mathrm{Y}$ & 7.8903250000 & 2832 & 6.0 & 5.4 & 0.100 & 0.098 \\
\hline 30 & $\mathrm{Y}$ & 7.8903125000 & 2831 & 6.1 & 5.4 & 0.100 & 0.097 \\
\hline 40 & $\mathrm{~N}$ & 7.8905375000 & 3115 & 4.7 & 4.3 & 0.040 & 0.039 \\
\hline 40 & $\mathrm{~N}$ & 7.8904500000 & 2987 & 5.0 & 4.5 & 0.072 & 0.070 \\
\hline 40 & $\mathrm{~N}$ & 7.8904375000 & 2982 & 5.1 & 4.6 & 0.073 & 0.071 \\
\hline 40 & $\mathrm{Y}$ & 7.8902875000 & 2812 & 5.6 & 5.0 & 0.113 & 0.110 \\
\hline 40 & $\mathrm{Y}$ & 7.8902875000 & 2803 & 5.6 & 5.0 & 0.116 & 0.113 \\
\hline 40 & $\mathrm{Y}$ & 7.8902875000 & 2795 & 5.6 & 5.0 & 0.118 & 0.115 \\
\hline 50 & $\mathrm{~N}$ & 7.8905375000 & 3055 & 4.5 & 4.1 & 0.059 & 0.058 \\
\hline 50 & $\mathrm{~N}$ & 7.8904500000 & 2955 & 4.8 & 4.3 & 0.085 & 0.083 \\
\hline 50 & $\mathrm{~N}$ & 7.8904375000 & 2934 & 4.9 & 4.4 & 0.090 & 0.088 \\
\hline 50 & $\mathrm{Y}$ & 7.8902750000 & 2768 & 5.4 & 4.9 & 0.130 & 0.126 \\
\hline 50 & $\mathrm{Y}$ & 7.8902750000 & 2759 & 5.4 & 4.9 & 0.132 & 0.129 \\
\hline 50 & $\mathrm{Y}$ & 7.8902750000 & 2758 & 5.4 & 4.9 & 0.133 & 0.129 \\
\hline
\end{tabular}

Table 6.5: Results from measuring sample a13 (polymer and undoped CdS)

\begin{tabular}{|c|c|c|c|c|c|c|c|}
\hline Temp ${ }^{\circ} \mathrm{C}$ & Light & $f_{o}(\mathrm{GHz})$ & $Q_{o}$ & $\epsilon_{r}{ }^{\prime}, t=9 \mu m$ & $\epsilon_{r}{ }^{\prime}, t=11 \mu m$ & $\tan \delta, t=9 \mu m$ & $\tan \delta, t=11 \mu m$ \\
\hline 30 & $\mathrm{~N}$ & 7.8924750000 & 3228 & 3.8 & 3.5 & 0.063 & 0.061 \\
\hline 30 & $\mathrm{~N}$ & 7.8923875000 & 3094 & 4.1 & 3.7 & 0.100 & 0.097 \\
\hline 30 & $\mathrm{~N}$ & 7.8923750000 & 3089 & 4.1 & 3.8 & 0.100 & 0.097 \\
\hline 30 & $\mathrm{Y}$ & 7.8922500000 & 2903 & 4.5 & 4.1 & 0.148 & 0.144 \\
\hline 30 & $\mathrm{Y}$ & 7.8922375000 & 2893 & 4.6 & 4.2 & 0.151 & 0.146 \\
\hline 30 & $\mathrm{Y}$ & 7.8922250000 & 2891 & 4.6 & 4.2 & 0.150 & 0.146 \\
\hline 40 & $\mathrm{~N}$ & 7.8924625000 & 3167 & 3.2 & 3.0 & 0.093 & 0.091 \\
\hline 40 & $\mathrm{~N}$ & 7.8923875000 & 3068 & 3.5 & 3.3 & 0.122 & 0.119 \\
\hline 40 & $\mathrm{~N}$ & 7.8923750000 & 3055 & 3.5 & 3.3 & 0.126 & 0.122 \\
\hline 40 & $\mathrm{Y}$ & 7.8922375000 & 2874 & 4.0 & 3.7 & 0.176 & 0.171 \\
\hline 40 & $\mathrm{Y}$ & 7.8922375000 & 2872 & 4.0 & 3.7 & 0.176 & 0.171 \\
\hline 40 & $\mathrm{Y}$ & 7.8922375000 & 2871 & 4.0 & 3.7 & 0.177 & 0.172 \\
\hline 50 & $\mathrm{~N}$ & 7.8924750000 & 3134 & 3.0 & 2.8 & 0.112 & 0.109 \\
\hline 50 & $\mathrm{~N}$ & 7.8923875000 & 3024 & 3.3 & 3.1 & 0.145 & 0.141 \\
\hline 50 & $\mathrm{~N}$ & 7.8923875000 & 3015 & 3.3 & 3.1 & 0.149 & 0.145 \\
\hline 50 & $\mathrm{Y}$ & 7.8922500000 & 2843 & 3.8 & 3.5 & 0.198 & 0.192 \\
\hline 50 & $\mathrm{Y}$ & 7.8922375000 & 2840 & 3.8 & 3.5 & 0.197 & 0.192 \\
\hline 50 & $\mathrm{Y}$ & 7.8922375000 & 2832 & 3.8 & 3.5 & 0.201 & 0.195 \\
\hline
\end{tabular}


Table 6.6: Results from measuring sample al4 (polymer and undoped CdS)

\begin{tabular}{|c|c|l|l|c|c|c|c|}
\hline Temp ${ }^{\circ} \mathrm{C}$ & Light & $f_{o}(\mathrm{GHz})$ & $Q_{o}$ & $\epsilon_{r}{ }^{\prime}, t=9 \mu m$ & $\epsilon_{r}{ }^{\prime}, t=11 \mu m$ & $\tan \delta, t=9 \mu m$ & $\tan \delta, t=11 \mu m$ \\
\hline 30 & $\mathrm{~N}$ & 7.8924125000 & 3171 & 4.6 & 4.2 & 0.069 & 0.067 \\
\hline 30 & $\mathrm{~N}$ & 7.8923625000 & 3109 & 4.8 & 4.3 & 0.083 & 0.081 \\
\hline 30 & $\mathrm{~N}$ & 7.8923625000 & 3106 & 4.8 & 4.3 & 0.084 & 0.082 \\
\hline 30 & $\mathrm{Y}$ & 7.8922375000 & 2937 & 5.3 & 4.7 & 0.123 & 0.119 \\
\hline 30 & $\mathrm{Y}$ & 7.8922250000 & 2943 & 5.3 & 4.7 & 0.120 & 0.117 \\
\hline 30 & $\mathrm{Y}$ & 7.8922250000 & 2943 & 5.3 & 4.7 & 0.120 & 0.117 \\
\hline 40 & $\mathrm{~N}$ & 7.8924250000 & 3184 & 4.0 & 3.7 & 0.074 & 0.072 \\
\hline 40 & $\mathrm{~N}$ & 7.8923625000 & 3092 & 4.2 & 3.9 & 0.098 & 0.095 \\
\hline 40 & $\mathrm{~N}$ & 7.8923500000 & 3087 & 4.3 & 3.9 & 0.099 & 0.096 \\
\hline 40 & $\mathrm{Y}$ & 7.8922375000 & 2920 & 4.7 & 4.2 & 0.141 & 0.137 \\
\hline 40 & $\mathrm{Y}$ & 7.8922500000 & 2918 & 4.6 & 4.2 & 0.143 & 0.139 \\
\hline 40 & $\mathrm{Y}$ & 7.8922125000 & 2917 & 4.8 & 4.3 & 0.140 & 0.136 \\
\hline 50 & $\mathrm{~N}$ & 7.8924500000 & 3151 & 3.7 & 3.4 & 0.090 & 0.088 \\
\hline 50 & $\mathrm{~N}$ & 7.8923750000 & 3055 & 4.0 & 3.7 & 0.116 & 0.112 \\
\hline 50 & $\mathrm{~N}$ & 7.8923750000 & 3035 & 4.0 & 3.7 & 0.122 & 0.119 \\
\hline 50 & $\mathrm{Y}$ & 7.8922375000 & 2892 & 4.5 & 4.1 & 0.156 & 0.152 \\
\hline 50 & $\mathrm{Y}$ & 7.8922375000 & 2882 & 4.5 & 4.1 & 0.159 & 0.155 \\
\hline 50 & $\mathrm{Y}$ & 7.8922375000 & 2874 & 4.5 & 4.1 & 0.162 & 0.157 \\
\hline
\end{tabular}

\subsection{Optical Material Property Characterization}

Transmission measurements were made on all the samples using the setup represented by Figure 6.3. The first measurements made were of the glass cover slips used. The optical transmission characteristics of the cover slips were measured at wavelengths from $400-800 \mathrm{~nm}$ and gave a $90 \%$ transmission across the entire band. These results match the light transmittance data given for the corning 0211 glass cover slips [ ], which confirms that the setup is functioning properly.

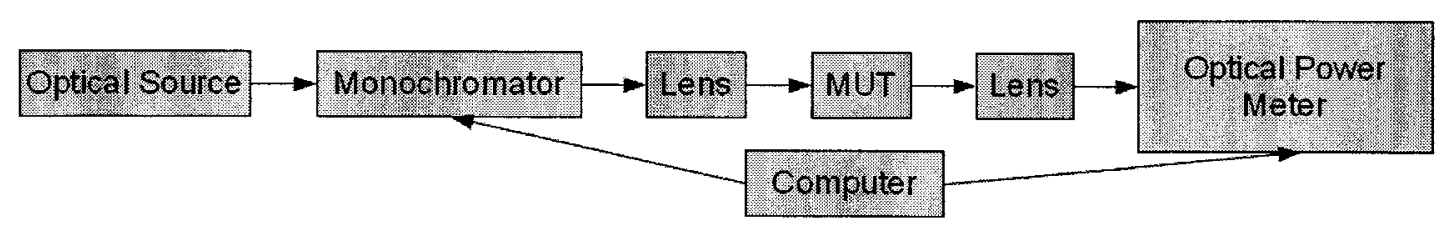

Figure 6.3: Setup used to measure optical transmission 
The transmittance of the materials were measured and the results are shown in Figures 6.4 and 6.5 .

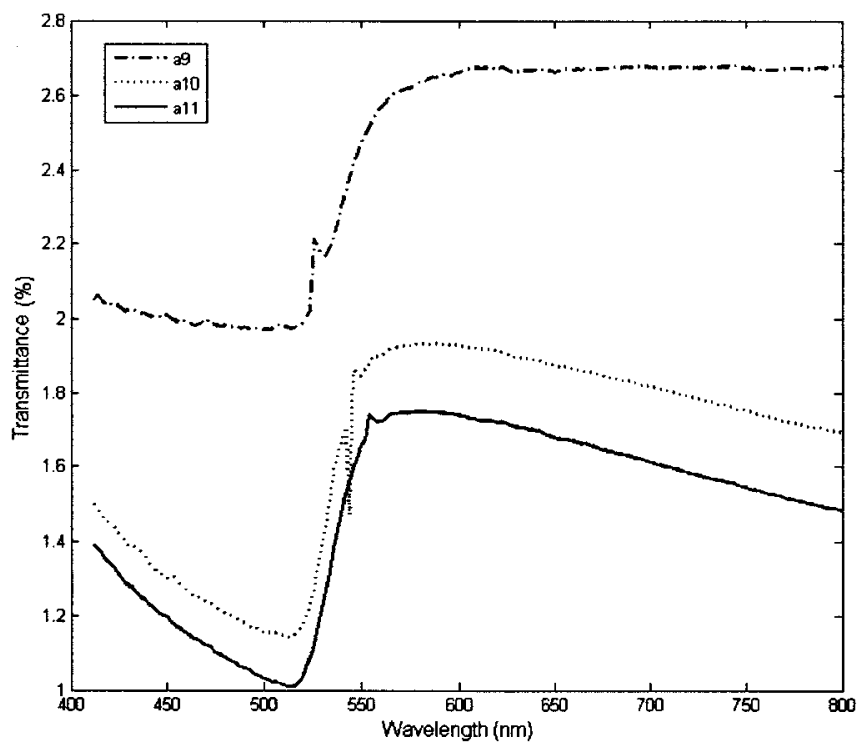

Figure 6.4: Optical transmittance of polymer and doped CdS 


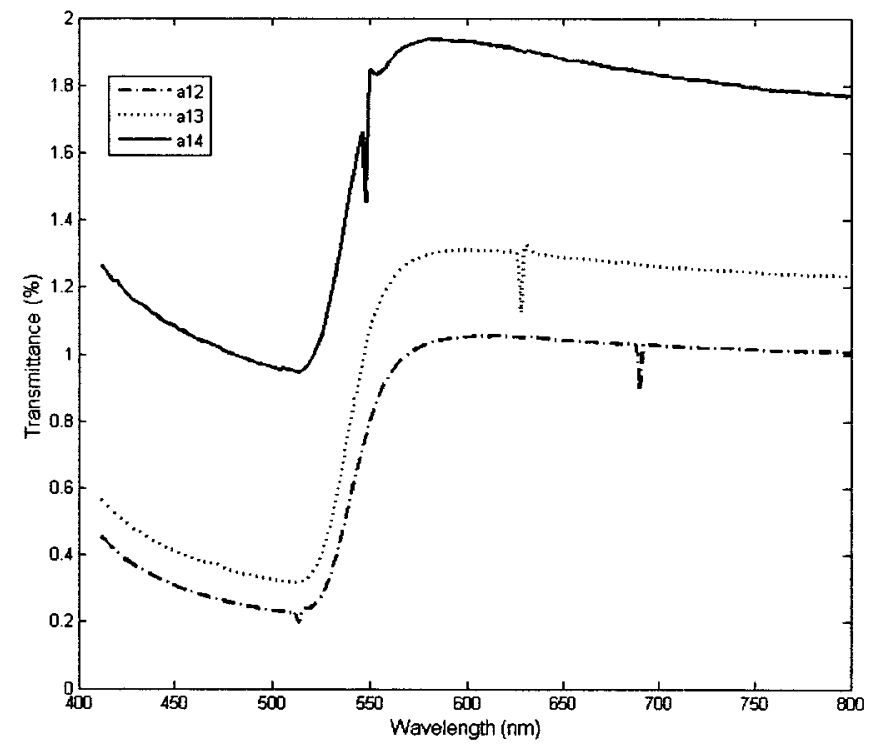

Figure 6.5: Optical transmittance of polymer and undoped CdS

\subsection{Summary}

This chapter presented the results from measuring the microwave and optical properties of the polymer-CdS films. The results are further analyzed and discussed in the next chapter. 


\section{Chapter 7}

\section{Analysis and Discussion of Findings}

\subsection{Introduction}

An optically tunable material is possible and may be practical for use in tunable microwave circuits. The data presented in this thesis demonstrates a material that shows an increase in the effective dielectric constant upon illumination. The remainder of this chapter discusses possible issues and the results.

\subsection{Optical Properties of Films}

The optical properties of the films must be investigated further since the results shown in Figures 6.4 and 6.5 fall short of expectations. However, some analysis of the results can be performed.

The optical transmission measurements show an increase in transmission around $520 \mathrm{~nm}$ as expected. However, the amount of transmitted light above $520 \mathrm{~nm}$ was much lower than the expected value between $40-80 \%$ transmitted [ , ], and could be due to many reasons. The method used does not allow for the reflected light to be measured. Also, for the transmission measurements the detector was far enough back 
from the sample that most of the scattered light may not be recorded. It is likely that the spherical inclusions scattered much of the light, thus preventing it from reaching the detector.

\subsection{Expected Dielectric Constant}

The dielectric constant of the polymer-CdS composite was expected to be around 6.6. This is because $72 \%$ of the material consisted of CdS which has an $\epsilon_{r}^{\prime}$ of 8.5 and mixture rules [ ] predict that the dielectric constant of the composite material to be around 6.6. This is assuming that the polymer has a dielectric constant of 3 and the CdS is not conductive. This value is higher than the value measured.

The measured value could be lower for many reasons, such as:

- Shape of CdS particles not perfectly spherical

- Volume fill fraction of CdS lower than $72 \%$

- Dielectric constant of polymer lower than 3

- Air gaps and voids in samples not accounted for

- Inaccuracies in thickness measurements or films are not uniform

The dielectric constants of the films measured should have been much closer to 6.6. In order to determine why the measured values are so much lower, the thickness of the films must be known to a higher degree of accuracy and the prevalence of air gaps must be eliminated, minimized, or accounted for. The thickness measurements are suspect because the dielectric values for all samples should have overlapped if the thickness of the materials was between 9-11 $\mu \mathrm{m}$. However, the possibility that some samples have larger or more air gaps than others cannot be ignored. Since there is 
a high uncertainty in the thickness of the films, only the relative shift in dielectric constant can be relied upon.

\subsection{Permittivity Changes with Optical Illumina- tion}

The complex permittivity of all the samples increased when illuminated as shown in Figures 7.1 and 7.2. From Figures 7.3 and 7.4, one can see that all the samples had a larger increase in the dielectric constant and dielectric loss tangent upon illumination the first time. For Figures 7.3 and 7.4, change 1 represents the first change in dielectric constant or dielectric loss tangent with optical illumination at a specific temperature point, while change 2 and 3 represent subsequent changes in materials properties with optical illumination at the same temperature point. This suggests that the material takes more than 10 seconds to return to steady state after illumination. For this reason only the first measurement with and without illumination at each temperature point is analyzed. Since the properties of the glass cover slips were only measured at $30^{\circ} \mathrm{C}$, only the measurements at that temperature point will be analyzed for changes in the complex permittivity of the materials. 


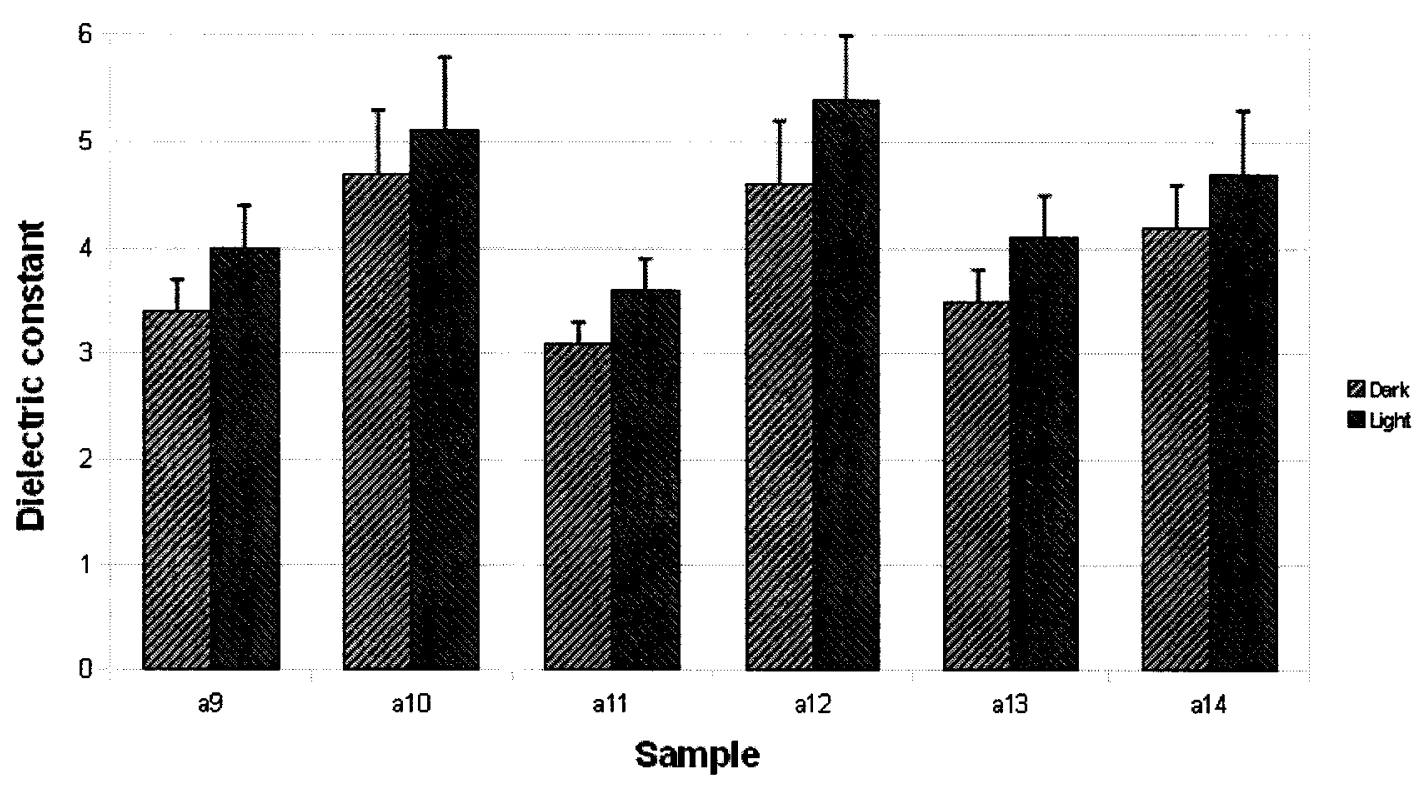

Figure 7.1: Effect of illumination on the dielectric constant of all samples at $30^{\circ} \mathrm{C}$ $(t=11 \mu \mathrm{m}$. Error bars represent $t=9 \mu \mathrm{m}$ samples $)$

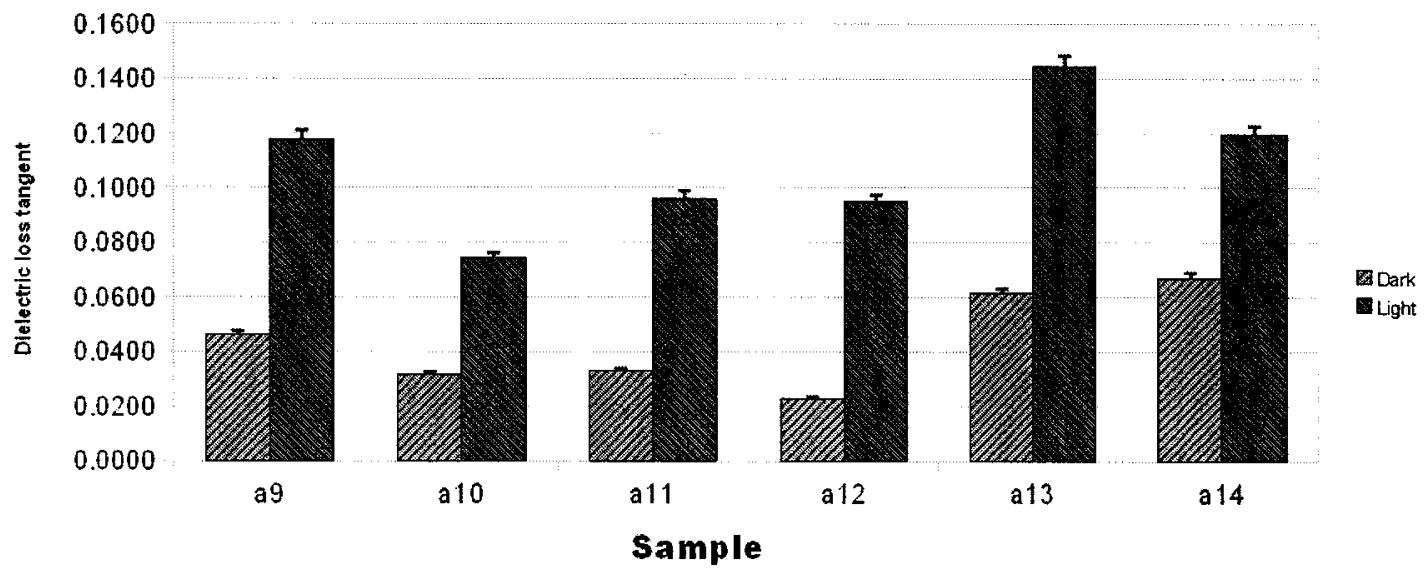

Figure 7.2: Effect of illumination on the dielectric loss tangent of all samples at $30^{\circ} \mathrm{C}(t=11 \mu \mathrm{m}$. Error bars represent $t=9 \mu \mathrm{m}$ samples $)$ 


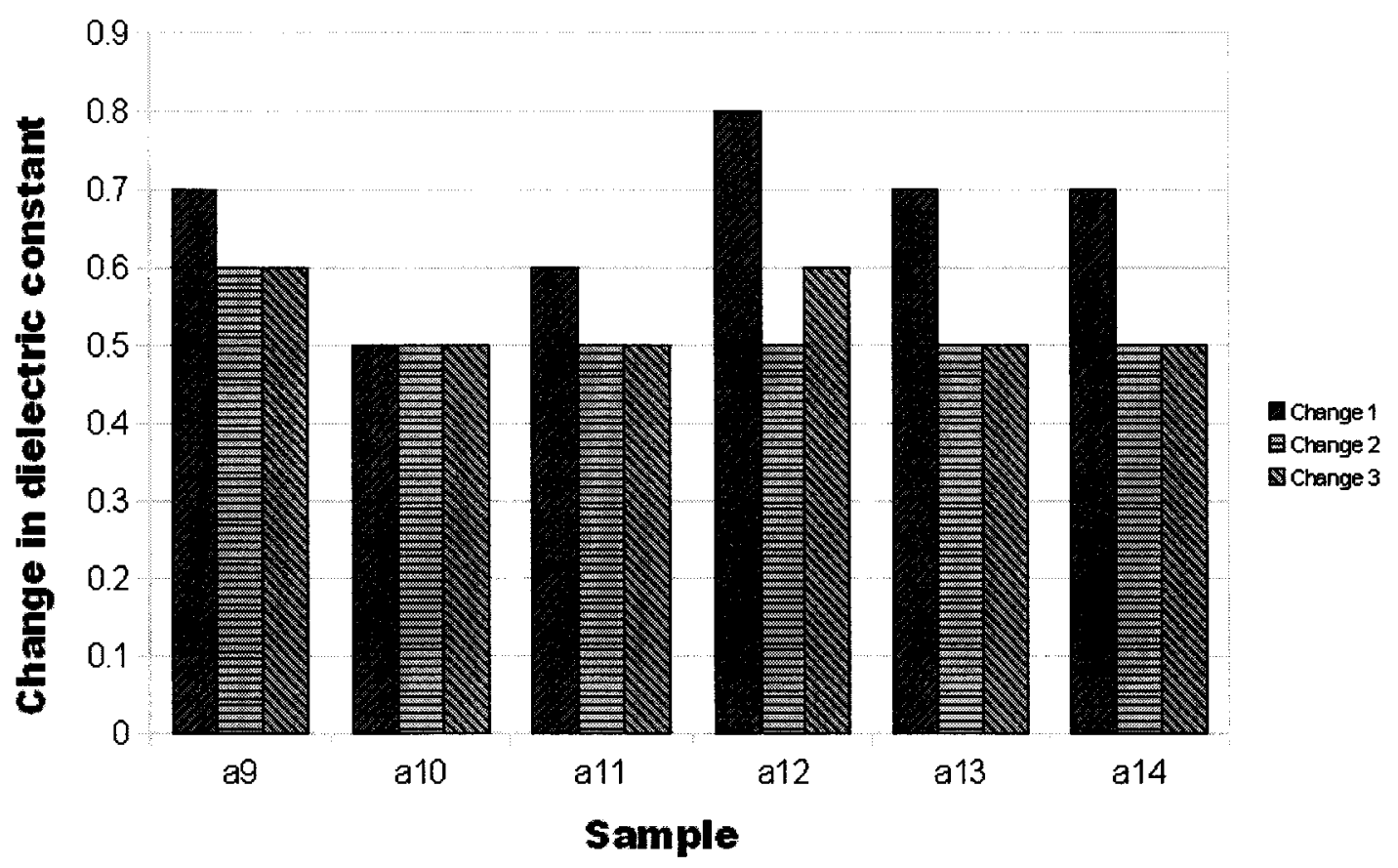

Figure 7.3: Change in dielectric constant with illumination

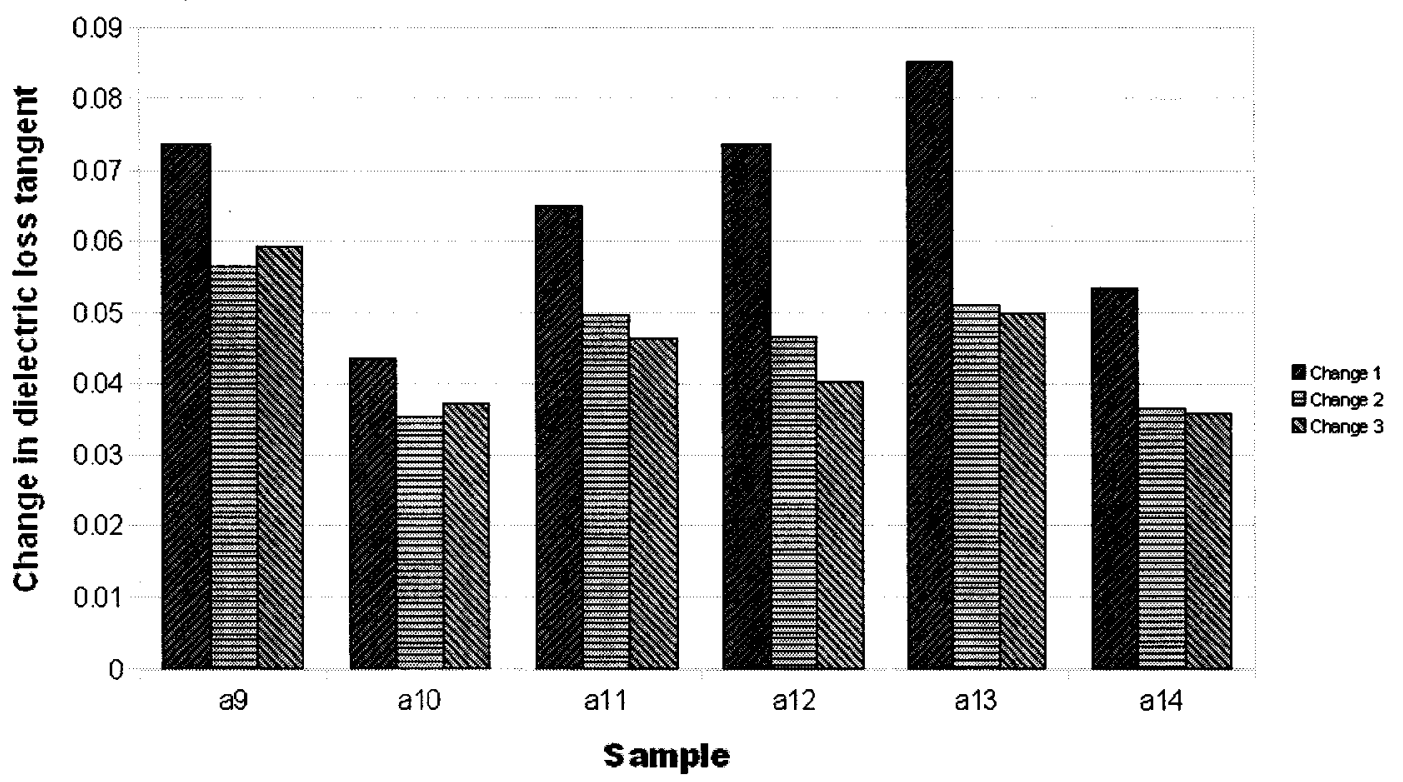

Figure 7.4: Change in dielectric loss tangent with illumination

The undoped samples (a12-a14) showed a larger change in dielectric constant and 
dielectric loss tangent than the doped samples (a9-a11). The undoped samples had a change in dielectric constant between $12-21 \%$, while the doped samples showed a change between 9-19\%. The undoped samples showed an increase in dielectric loss tangent between 0.05-0.09, while the doped samples showed and increase between 0.04-0.07. It is possible that these values are off by up to 15 percent due to uncertainty in the thickness measurements of the films. However, uncertainty in thickness measurements does not change the fact that the complex permittivity of the materials increased with illumination.

The increase in dielectric constant could not be predicted with much accuracy for this work. This was due to the fact that the electrical transport and electron-hole-pair lifetimes for the material are not known, therefore the bulk properties of CdS must be assumed. The optical transmission measurements do not give an accurate indication of how much of the light is absorbed at each wavelength. The optical power of the light source is not known versus wavelength with much accuracy, only the optical power spectrum of the lamp provided by the manufacturer can be used. However, the calculation performed in Section 3.2 shows that if all the power were absorbed then the conductivity of the particles would increase by $10 \mathrm{~S} / \mathrm{m}$. It is likely that only a tenth of the optical power or less is being absorbed. However, even this level of absorption is expected to cause an increase in dielectric constant.

\subsection{Temperature Effects}

One possible source of changes in dielectric constant that must be addressed is a increase in temperature due to illumination. It is unlikely that the light source provided enough power to cause an increase in temperature high enough to increase the dielectric constant of any of the materials. However, even if the light source 
heated up the samples, the dark dielectric constant of the films decreased with increasing temperature as shown in Figure 7.5, and the dark dielectric loss tangent of the films increased with increasing temperature as shown Figure 7.6. This demonstrates that temperature increases would not increase the effective dielectric constant of the materials.

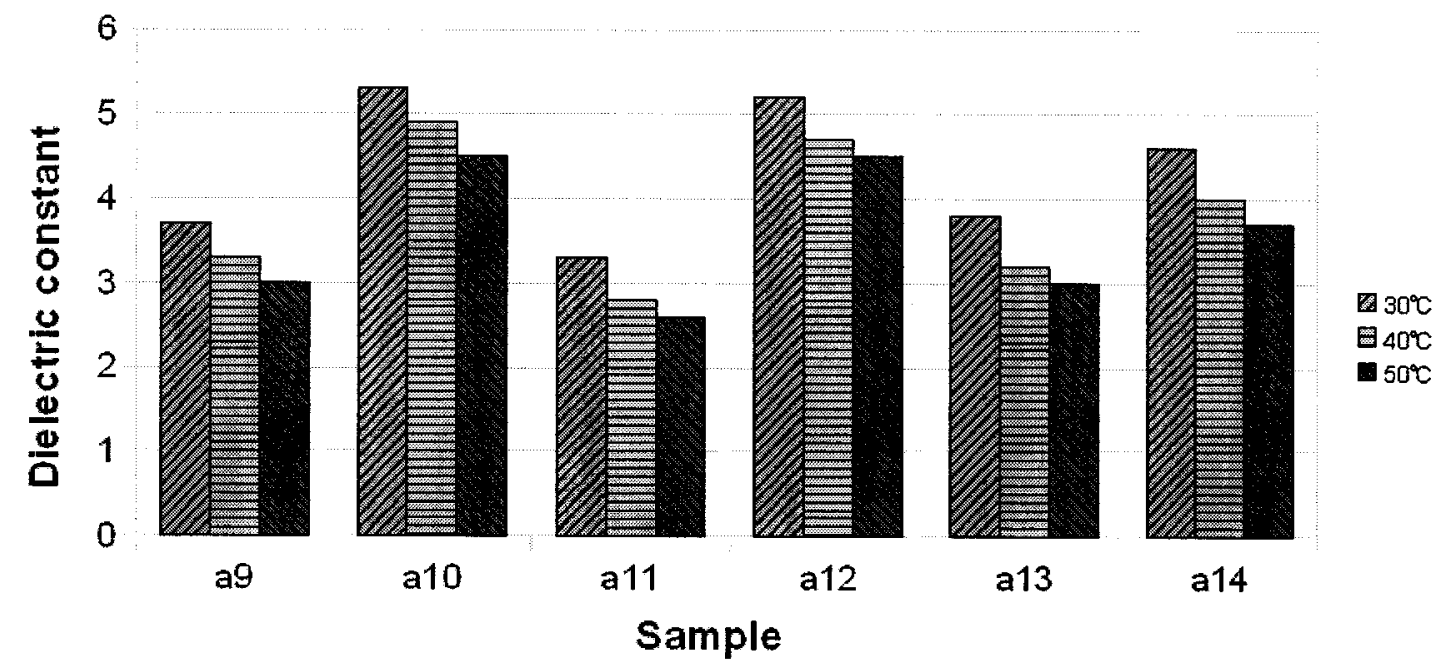

Figure 7.5: Dielectric constant of samples at different temperatures without illumination 


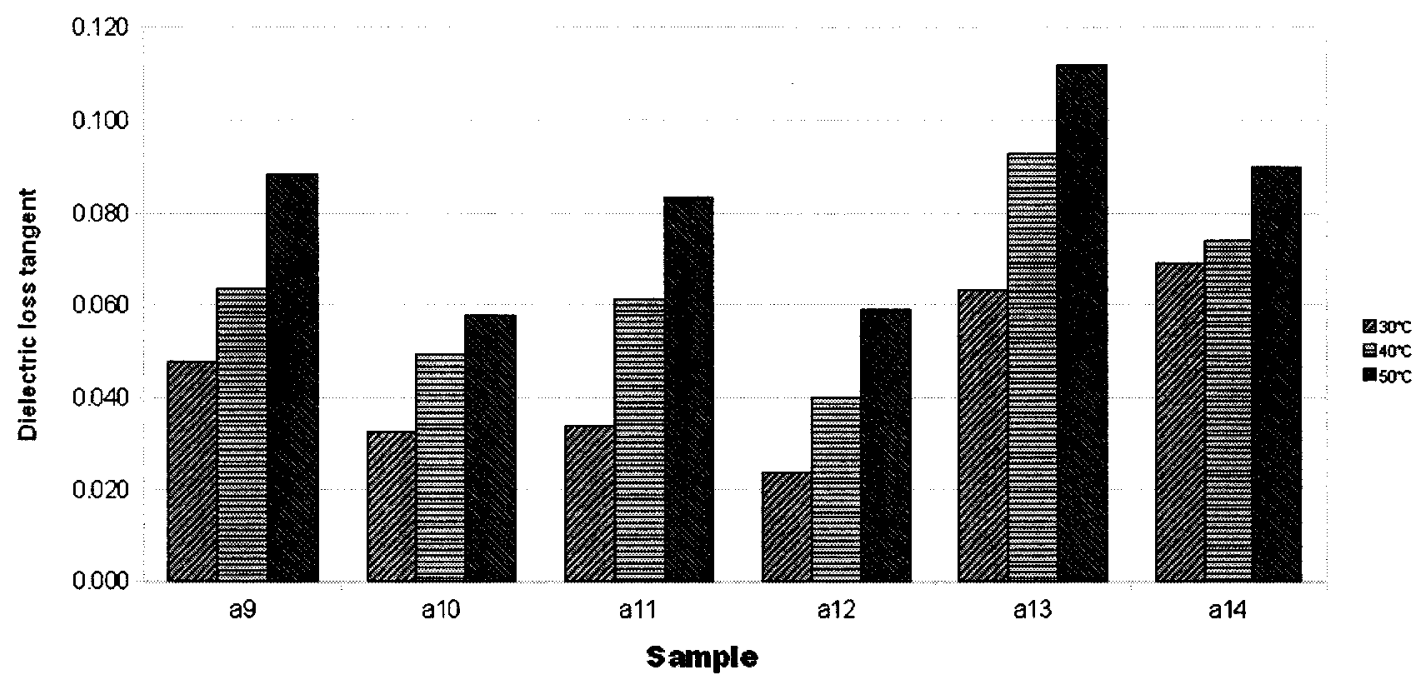

Figure 7.6: Dielectric loss tangent of samples at different temperatures without illumination

The change in the effective dielectric constant and the effective dielectric loss tangent with optical illumination at different temperature points is shown in Figures 7.7 and 7.8. This shows that temperature has no significant effect on changes in the dielectric constant and the dielectric loss tangent of the materials with optical illumination. 


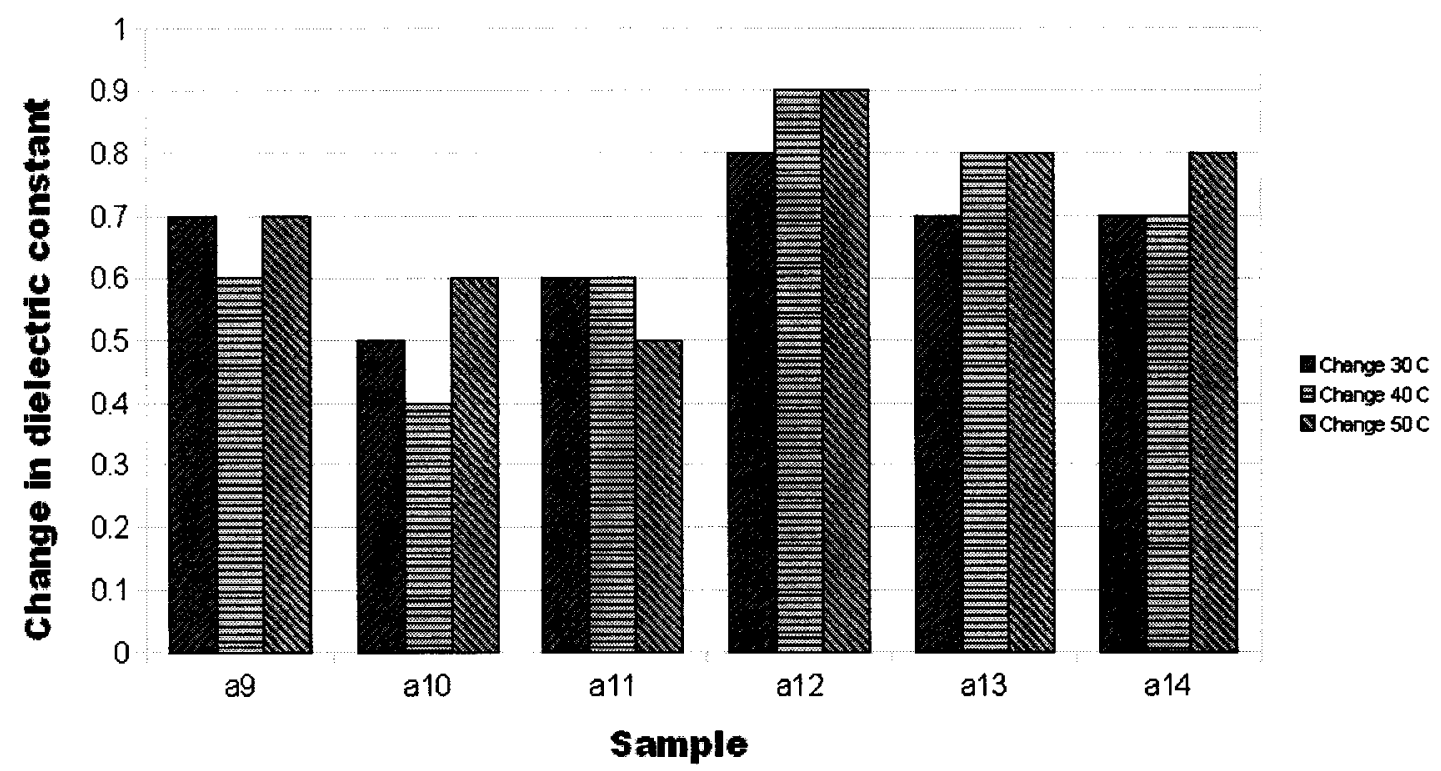

Figure 7.7: Change in dielectric constant of samples at different temperatures with illumination

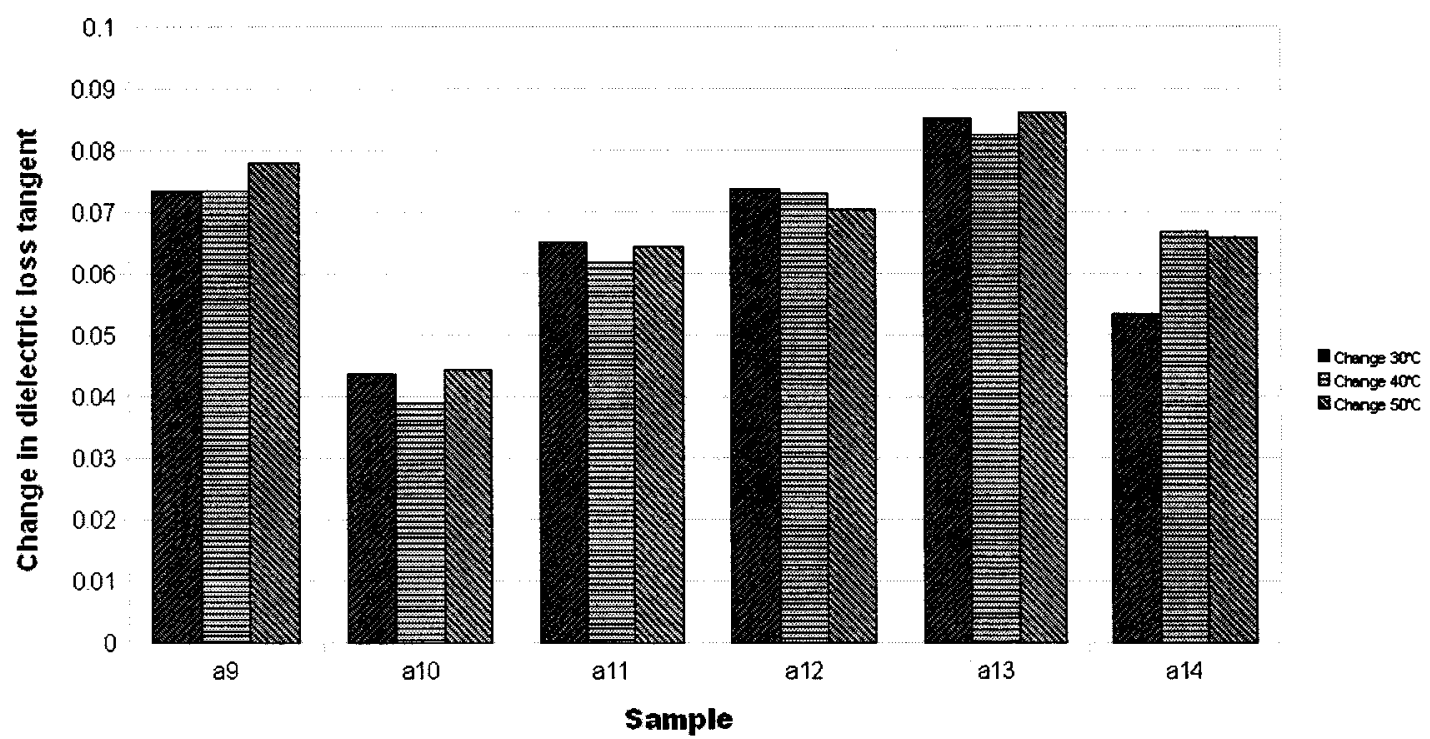

Figure 7.8: Change in dielectric loss tangent of samples at different temperatures with illumination 


\subsection{Appropriateness of an Effective Dielectric Constant}

The effective dielectric constant of the material cannot be considered isotropic. The orientation of the electric field of the measurement apparatus allows for an effective dielectric constant to be used because the electric field was tangential to the sample. However, if the electric field was normal to the sample, then the sample would be too thin to use an effective dielectric constant due to surface effects [ , ]

Using an effective dielectric constant for the whole material when the electric field is orientated parallel to the sample may not be appropriate either. If the conductivity of all the particles is the same when illuminated then an effective dielectric constant for the entire material is appropriate. However, if the conductivity is a function of depth from the surface, then the effective dielectric constant would also have to be a function of depth. This issue needs to be explored further.

\subsection{Comparison to Ferroelectric Materials}

The dielectric tunability of the materials presented in this work is nearly the same as that demonstrated for some ferroelectric materials, but is lower than the best values reported. The materials presented in this work were not optimized. Simulations from Section 3.3 suggest that materials could be created that have tunability of $26 \%$, and possibly greater. The materials presented in the previous chapter had higher dielectric loss than is practical, however simulations also suggest this can be lowered well below that of most ferroelectric materials by limiting inter-particle contact. Overall, optically tunable dielectric materials have the potential to be a viable tunable material. 


\subsection{Simulating the Composite Material}

Unfortunately the measured results cannot be directly compared to simulations. The volume fill fraction of cadmium sulfide particles was $72 \%$, which is higher than the maximum of $50 \%$ volume fill fraction that can be simulated. This is because simulations assume all the particles are the same size $(1 \mu m)$, while the CdS particles are a range of sizes, which allows more volume to be filled. Also, the electrical properties properties of both the polymer and CdS are not known with enough accuracy. The films must be more uniform and the prominence of voids and gaps must be known. For all these reasons attempting to simulate the material measured was not appropriate. 


\section{Chapter 8}

\section{Conclusions and Recommendations}

\subsection{Summary of Work}

The thesis presented an analytical and numerical basis for proposing a novel optically tunable dielectric material. The anyalytical basis was based on Bruggeman's effective medium approximation and percolation theory. Bruggeman's effective medium approximation predicts that adding conductive spherical particles causes the effective dielectric constant to increase as more particles are added. However, this approximation breaks down once a large enough volume of particles is added. At this point, what is known as percolation theory becomes useful to estimate the effect of adding more conductive particles. This theory shows that once a sufficient volume of conductive particles is added to the material, adding more particles will cause a rapid increase in the effective dielectric constant of the material. Analytical approaches are limited and a more robust method must be used for analyzing composite materials. A numerical approach using HFSS was presented and the results compared well with that predicted by Bruggeman's effective medium approximation and percolation theory.

Next, a novel microwave material characterization apparatus designed specifically 
for measuring the material properties of an optically tunable dielectric material was presented. This apparatus was based on a material characterization apparatus known as the split post dielectric resonator. The apparatus was able to accurately measure the microwave properties of films and substrate materials. The material characterization apparatus has a removable lid in order to allow light to be applied to the samples being measured.

Finally, the thesis was concluded with the presentation and discussion of the results from measuring a novel optically tunable dielectric material. Two similar materials deposited on glass cover slipes were measured using the microwave material characterization apparatus developed in this work. Both materials were made of $72 \%$ by volume of CdS and a transparent polymer. One of the samples used CdS doped with copper, while the other sample was undoped. Both types of materials showed an increase in the effective dielectric constant and effective dielectric loss tangent with optical illumination. Measuring the effective dielectric constant of the materials in the dark over a range of temperatures showed that the changes were not due to thermal effects. The materials showed tunabilities of at least $9 \%$, however the dielectric loss tangent of the material was as high as 0.148 for some of the samples.

\subsection{Further Work}

This thesis demonstrates that there is much more work to do in the area of optically tunable dielectrics. Firstly, the method presented to predict the properties of materials must be developed further. Materials with filler particles with different shapes, sizes, and orientations must be modeled in simulations. Then the materials must be fabricated and measured. The materials must be made of constituents with known properties to allow for proper comparison. 
Once the effective properties of materials can be simulated effectively, photoconductive particles must be designed using simulation. In simulation the effect of particle size and shape can be investigated. Also, the effect of adding a shell to the particle to minimize inter-particle interactions can be investigated. Once the desired properties of the particle are known, real materials can be determined and the particles can be made.

The optical properties of the films must also be investigated. It is necessary to know how light interacts with the photoconductive particles. How much light is being reflected, transmitted, and absorbed must be known. Furthermore, the use of a shell on the photoconductive particles in order to decrease reflections must be investigated. Lastly, the effect of polarized and unpolarized light on the material must be investigated.

The material characterization apparatus could benefit from additional work. Effects of humidity/temperature must be eliminated or accounted for. An exhaustive accuracy analysis on the cavity would also be beneficial. Also, an apparatus to determine the response time of the material must be designed.

There is much more work to do on the materials proposed in this work. They appear to be a viable approach to making optically tunable microwave devices. 


\section{List of References}

[1] K. C. Kao, Dielectric Phenomena In Solids. Elsevier, 2004.

[2] L. F. Chen, C. K. Ong, C. Neo, V. V. Varadan, and V. K. Varadan, Microwave Electronics: Measurement and Materials Characterization. John Wiley \& Sons, 2004.

[3] D. Stauffer and A. Aharony, Introduction to Percolation Theory. Taylor \& Francis, 1994.

[4] Q. Xue, "The influence of particle shape and size on electric conductivity of metal-polymer composites," European Polymer Journal, vol. 40, pp. 323-327, 2004.

[5] S. W. Liu, J. Weaver, Z. Yuan, W. Donner, and C. L. Chen, "Ferroelectric (pb, sr)tio3 epitaxial thin films on (001) mgo for room temperature high-frequency tunable microwave elements," Applied Physics Letters, vol. 87, pp. 142905-1142905-3, 2005.

[6] W. Chang, S. W. Kirchoefer, J. M. Pond, J. A. Bellotti, and S. B. Qadri, "Roomtemperature tunable microwave properties of strained srtio3," Journal of Applied Physics, vol. 96, pp. 6629-6633, 2004.

[7] M. Jain, N. K. Karan, R. S. Katiyar, A. S. Bhalla, F. A. Miranda, and F. W. V. Keuls, " $\mathrm{Pb}_{0} .3 \mathrm{sr}_{0} .7 \mathrm{tio}_{3}$ thin films for high-frequency phase shifter applications," Applied Physics Letters, vol. 85, pp. 275-277, 2004.

[8] Skyworks, "Appl. note: Introduction and applications for temperature stable dielectric resonators," 2007.

[9] Skyworks, "Appl. note 851: Tuning and exciting dielectric resonator modes," 2007. 
[10] J. Krupka, "Frequency domain complex permittivity measurements at microwave frequencies," Measurement Science and Technology, vol. 17, pp. R55-R70, 2006.

[11] J. Krupka, "Measurement of the complex permittivity of metal nanoislands and the surface resistance of thin conducting films at microwave frequencies," Measurement Science and Technology, vol. 19, pp. 1-8, 2008.

[12] D. Kajfez and E. J. Hwan, "Q-factor measurement with network analyzer," IEEE Transactions on Microwave Theory and Techniques, vol. 7, pp. 666-669, 1984.

[13] Newport, "Oriel quartz tungsten halogen lamps." datasheet.

[14] C. Musoll-Anguiano, I. Llamas-Garro, Z. Brito-Brito, L. Pradell, and A. CoronaChavez, "Fully adaptable band-stop filter using varactor diodes," Microwave and Optical Technology Letters, vol. 52, pp. 554-558, 2010.

[15] F. Svelto, P. Erratico, S. Manzini, and R. Castello, "A metal-oxidesemiconductor varactor," IEEE Electron Device Letters, vol. 20, pp. 164-166, 1999.

[16] S. S. Gevorgian and E. L. Kollberg, "Do we really need ferroelectrics in paraelectric phase only in electrically controlled microwave devices," IEEE Transactions on Microwave Theory and Techniques, vol. 49, pp. 2117-2124, 2001.

[17] A. Tomback, J.-P. Maria, F. Ayguavives, Z. Jin, G. T. Stauf, A. I. Kingon, and A. Mortazawi, "Tunable barium strontium titanate thin film capacitors for $\mathrm{rf}$ and microwave applications," IEEE Microwave and Wireless Components Letters, vol. 12 , pp. 3-5, 2002.

[18] M. Bakri-Kassem and R. R. Mansour, "Linear bilayer ald coated mems varactor with high tuning capacitance ratio," Journal of Microelectromechanical Systems, vol. 18, pp. 147--153, 2009.

[19] M. Rais-Zadeh, P. A. Kohl, and F. Ayazi, "Mems switched tunable inductor," Jounal of Microelectromechanical Systems, vol. 17, pp. 78-84, 2008.

[20] G. Qiu, C. S. Tsai, B. S. T. Wang, and Y. Zhu, "A yig/ggg/gaas-based magnetically tunable wideband microwave band-pass filter using cascaded band-stop filters," IEEE Transactions on Magnetics, vol. 44, pp. 3123--3126, 2008. 
[21] S. I. M. Sheikh, A. A. P. Gibson, M. Basorrah, G. Alhulwah, K. Alanizi, M. Alfarsi, and J. Zafar, "Analog/digital ferrite phase shifter for phased array antennas," IEEE Antennas and Wireless Propogation Letters, vol. 9, pp. 319-321, 2010 .

[22] H.-P. Change, J. Qian, B. A. Cetiner, F. D. Flaviis, M. Backman, and G. P. $\mathrm{Li}$, "Rf mems switch fabricated on microwave-laminate printed circuit boards," IEEE Electron Device Letters, vol. 24, pp. 227-229, 2003.

[23] A. Karabegovic, R. M. O'Connell, and W. C. Nunally, "Photoconductive switch design for microwave applications," IEEE Transactions on Dielectrics and Electrical Insulation, vol. 16, pp. 1011-1019, 2009.

[24] B. Boyer, J. Haider, A. Vilcot, and M. Bouthinon, "Tunable microwave load based on biased photoinduced plasma in silicon," IEEE Transactions on Microwave Theory and Techniques, vol. 45, pp. 1362-1367, 1997.

[25] M. R. Chaharmir, J. Shaker, M. Cuhaci, and A.-R. Sebak, "Novel photonicallycontrolled reflectarray antenna," IEEE Transactions on Antennas and Propagation, vol. 54, pp. 1134-1141, 2006.

[26] D. M. Pozar, Microwave Engineering, 3rd ed. John Wiley \& Sons, 2004.

[27] T. Blythe and D. Bloor, Electrical Properties of Polymers, 2nd ed. Cambridge University Press, 2005.

[28] L. Solymar and D. Walsh, Electrical Properties of Materials, 8th ed. Oxford University Press, 2008.

[29] C. Brosseau, "Modelling and simulation of dielectric heterostructures: A physical survey from an historic perspective," Journal of Physics D: Applied Physics, vol. 39, pp. 1277-1294, 2006.

[30] L. Jylha and A. Sihvola, "Equation for the effective permittivity of particlefilled composites for material design applications," Journal of Physics D: Applied Physics, vol. 40, pp. 4966-4973, 2007.

[31] D. A. G. Bruggeman, "Dielectric constant and conductivity of mixtures of isotropic materials," Ann. Phys. (Leipzig), vol. 24, pp. 636-664, 1935. 
[32] D. S. McLachlan, "Measurement and analysis of a model dual-conductivity medium using a generalized effective-medium theory," J. Phys. C: Solid State Phys., vol. 21, pp. 1521-1532, 1988.

[33] D. S. McLachlan, "The complex permittivity of emulsions: an effective mediapercolation equation," Solid State Communications, vol. 72, pp. 831-834, 1989.

[34] T. K. H. Starke, C. Johnston, S. Hill, P. Dobson, and P. S. Grant, "The effect of inhomogeneities in particle distribution on the dielectric properties of composite films," J. Phys. D: Appl. Phys., vol. 39, pp. 1305-1311, 2006.

[35] C. Brosseau, P. Queffelec, and P. Talbot, "Microwave characterization of filled polymers," Journal of Applied Physics, vol. 89, pp. 4532-4540, 2001.

[36] S. O. Kasap, Principles of Electronic Materials and Devices, 3rd ed. McGrawHill, 2006.

[37] A. J. Seeds and A. A. A. D. Salles, "Optical control of microwave semiconductor devices," IEEE Transactions on Microwave Theory and Techniques, vol. 38, pp. 577-585, 199.

[38] L. B. Kong, S. Li, T. S. Zhang, J. W. Zhai, F. Y. C. Boey, and J. Ma, "Electrically tunable dielectric materials and strategies to improve their performance," Progress in Materials Science, pp. 840-893, 2010.

[39] L. Yan, L. Kong, L. Chen, K. Chong, C. Y. Tan, and C. K. Ong, " $\mathrm{Ba}_{0.5} \mathrm{Sr}_{0.5} \mathrm{tio}_{3}$ $\mathrm{bi}_{1.5} \mathrm{Zn}_{1.0} \mathrm{nb}_{1.5} \mathrm{O}_{7}$ composite thin films with promising microwave dielectric properties for microwave device applications," Applied Physics Letters, vol. 85, pp. $3522-3524,2004$.

[40] T. Morolari, "Characterization of optically sensitive polymer and application to microwave antenna," Master's thesis, Carleton University, 2009.

[41] O. Madelung, Semiconductors: Data Handbook 3rd ed. Springer, 2004.

[42] A. Valyomana, K. P. Vijayakumar, and C. Purushothaman, "Influence of annealing on lifetime of minority carriers in n-type cds films," Journal of Materials Science Letters, vol. 11, pp. 76-78, 1992.

[43] J. Sheen, "Comparison of microwave dielectric property measurements by transmission/reflection techniques and resonance techniques," Measurement Science and Technology, vol. 20, pp. 1-12, 2009. 
[44] Skyworks, "Appl. note 821: Introduction to dielectrics," 2007.

[45] Skyworks, "Appl. note: Properties, test methods and mounting," 2007.

[46] J. Krupka, A. P. Gregory, O. C. Rochard, R. N. Clarke, B. Riddle, and J. BakerJarvis, "Uncertainty of complex permittivity measurements by split-post dielectric resonator technique," Journal of the European Ceramic Society, vol. 21, pp. 2673-2676, 2001.

[47] Skyworks, "8300 series: Temperature stable resonators," 2007. datasheet.

[48] Skyworks, "Dielectric and alumina supports," 2007. datasheet.

[49] Henkel, "Loctite 409," 2008. datasheet.

[50] Corning, "Corning 0211 microsheet: Product information," 2006. datasheet.

[51] I. Ted Pella, "Corning 0211 glass coverslips technical data." http://www . tedpella.com/histo_html/coverslip-info.htm, Aug. 2010.

[52] J.-H. Lee, J.-S. Yi, K.-J. Yang, J.-H. Park, and R.-D. Oh, "Electrical and optical properties of boron doped cds thin films prepared by chemical bath deposition," Thin Solid Films, vol. 431-432, pp. 344-348, 2003.

[53] K. Senthil, D. Mangalaraj, S. K. Narayandass, and S. Adachi, "Optical constants of vacuum-evaporated cadmium sulphide thin films measured by spectroscopic ellipsometry," Materials Science and Engineering B, vol. B78, pp. 53-58, 2000.

[54] J. Sheen, Z.-W. Hong, W. Liu, W.-L. Mao, and C.-A. Chen, "Study of dielectric constants of binary composites at microwave frequency by mixture laws derived from three basic particle shapes," European Polymer Journal, vol. 45, pp. 1316$1321,2009$.

[55] A. P. Vinogradov, "On the clausius-mossotti-lorenz-lorentz formula," Physica A, vol. 241 , pp. $216-222,1997$.

[56] S. Tretyakov, Analytical Modeling in Applied Electromagnetics. Artch House, 2003. 